\title{
ON NICHOLS ALGEBRAS OVER BASIC HOPF ALGEBRAS
}

\author{
NICOLÁS ANDRUSKIEWITSCH AND IVÁN ANGIONO
}

\begin{abstract}
This is a contribution to the classification of finite-dimensional Hopf algebras over an algebraically closed field $\mathbb{k}$ of characteristic 0 . Concretely, we show that a finite-dimensional Hopf algebra whose Hopf coradical is basic is a lifting of a Nichols algebra of a semisimple Yetter-Drinfeld module and we explain how to classify Nichols algebras of this kind. We provide along the way new examples of Nichols algebras and Hopf algebras with finite Gelfand-Kirillov dimension.
\end{abstract}

\section{CONTENTS}

1. Introduction

1.1. The context

1.2. Nichols algebras over basic Hopf algebras

1.3. The main result

1.4. Decompositions

2. Nichols algebras from decomposable braided vector spaces

2.1. Preliminaries

2.2. Nichols algebras

2.3. The coinvariant Nichols algebra

2.4. Nichols algebras of semisimple Yetter-Drinfeld modules

3. Semisimplicity and the diagram

3.1. Nichols algebras of graded or filtered Yetter-Drinfeld modules

3.2. Pointed Hopf algebras

3.3. Semisimplicity

3.4. The diagram

4. Decompositions from diagonal type 24

4.1. Generalities 24

4.2. Dimension $W=2$

4.3. Cartan type $A_{\theta}, \theta>2$

4.4. Cartan type $B_{\theta}, \theta>2$

4.5. Cartan type $C_{\theta}, \theta>2$

4.6. Cartan type $D_{\theta}, \theta>3$

5. Decompositions with a block 36

2010 Mathematics Subject Classification. 16T05.

The work of N. A. and I. A. was partially supported by CONICET, Secyt (UNC), the MathAmSud project GR2HOPF. 
5.1. A block and a point, weak interaction

5.2. A block and a point, mild interaction

\section{INTRODUCTION}

1.1. The context. We fix an algebraically closed field $\mathbb{k}$ of characteristic 0 . Let $\mathrm{H}$ be a Hopf algebra with bijective antipode, $\mathrm{H}_{0}$ its coradical (the sum of its simple subcoalgebras) and $\mathrm{H}_{[0]}$ its Hopf coradical (the subalgebra generated by $\mathrm{H}_{0}$, which is a Hopf subalgebra). The problem of the classification of those $\mathrm{H}$ with finite Gelfand-Kirillov dimension can be organized in four different classes according to the following conditions, cf. [AC]:

(a) $\mathrm{H}=\mathrm{H}_{0}$. That is, the class of cosemisimple Hopf algebras.

(b) $\mathrm{H}=\mathrm{H}_{[0]} \neq \mathrm{H}_{0}$, the class of (non-cosemisimple) Hopf algebras generated by the coradical.

(c) $\mathrm{H} \neq \mathrm{H}_{[0]}=\mathrm{H}_{0}$, i. e. the coradical is a (proper) Hopf subalgebra.

(d) $\mathrm{H} \neq \mathrm{H}_{[0]} \neq \mathrm{H}_{0}$.

There is no general method, to our knowledge, for the classes (a) and (b), even for the sub-problem of the classification of finite-dimensional Hopf algebras. For class (c) there is a well-known method [AS3] that was applied under various natural hypothesis; see $\mathrm{A2}, \mathrm{AS3}, \mathrm{AnG}$ and references therein.

Here we contribute to class (d) for the sub-problem of finite dimension, according to the method proposed in $\mathrm{AC}$ that extends AS3. Namely the graded Hopf algebra gr $\mathrm{H}$ associated to the standard filtration splits as $R \# \mathrm{H}_{[0]}$, where $R=\oplus_{n \geq 0} R^{n}$ is a graded Hopf algebra in the category of Yetter-Drinfeld modules over $\mathrm{H}_{[0]}$ (called the diagram). The proposal of AC is to classify the possible $R$ and then to compute all liftings (Hopf algebra deformations) of $R \# \mathrm{H}_{[0]}$. Our main result, Theorem 1.3 , answers fairly completely the first question under the assumption that $\mathrm{H}_{[0]}$ is basic with abelian group of characters $G$. Namely, $R$ should be the Nichols algebra of a semisimple Yetter-Drinfeld module and the list of such Nichols algebras is controlled by the classification of the finite-dimensional Nichols algebras over $G$ which follows from [H2].

The proof of Theorem 1.3 has three parts. The first one deals with Nichols algebras of semisimple Yetter-Drinfeld modules, see Theorem 1.1. In the second part, we prove that the Nichols algebra of a non-semisimple YetterDrinfeld module has infinite dimension, see Theorem 1.2.

For the last part, we notice that in general it is not known whether an arbitrary $R=\oplus_{n \geq 0} R^{n}$ arising from the standard filtration is coradically graded, or whether the subalgebra $R$ of $R$ generated by $\mathcal{Z}=R^{1}$ is a Nichols algebra, albeit $\mathscr{B}(\mathcal{Z})$ is a quotient of $\mathrm{R}$. Assuming that $R$ is finite-dimensional over $L$ basic with abelian group, we show that $R$ is a Nichols algebra in 
Subsection 3.4, the proof relies strongly on the description of liftings of Nichols algebras over abelian groups AnG, AnG2].

The smallest example of a non-semisimple Hopf algebra $L$ generated by its coradical was first considered by Radford; it has dimension 8 and is basic, being the dual of the pointed Hopf algebra $\mathbf{r}(-1)$, which is a lifting of a quantum line. The study of the Nichols algebras over $L=\mathbf{r}(-1)^{*}$ was undertaken in GG]: our Corollary 2.13 generalizes [GG, Theorem A]. We mention that one of the motivations of this paper was to put the results presented in GG in a general context. Nichols algebras over other basic, non-semisimple, Hopf algebras of small dimension were considered in $\mathrm{HX}$, X1, X2, X3].

Actually, the class of basic Hopf algebras is a source of examples of Hopf algebras generated by its coradical, perhaps the only understandable presently (up to routine modifications like tensoring with a semisimple one or passing to a Morita equivalent one). However, not every basic Hopf algebra has this property.

Question 1. Given a basic Hopf algebra $L, \operatorname{dim} L<\infty$, determine when it is generated by its coradical in terms of the deformation parameters of $B=L^{*}$.

1.2. Nichols algebras over basic Hopf algebras. Let $L$ be a finitedimensional basic Hopf algebra; that is every simple $L$-module has dimension 1 or equivalently $B=L^{*}$ is pointed. It was conjectured that such $B$ should be generated by group-like and skew-primitive elements [AS2]; the conjecture is valid in all known examples, e. g. when the group $G(B)$ is abelian

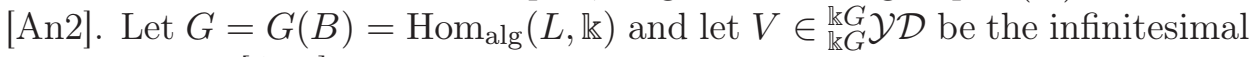
braiding of $B$ AS3]. We assume that

$$
\begin{aligned}
K & :=\operatorname{gr} B \simeq \mathscr{B}(V) \# \mathbb{k} G, \\
B & \text { is a cocycle deformation of gr } B .
\end{aligned}
$$

Hypothesis (1.1) is a rephrasing of the above Conjecture; in turn (1.2) again holds when $G$ is abelian $\mathrm{AnG}$ and in all known cases $\mathrm{GM}$.

In some instances, we shall also need that our finite group $G$ satisfies:

$$
\text { Every finite dimensional pointed Hopf algebra with group } G \text { is }
$$$$
\text { generated by group-like and skew-primitive elements. }
$$

Evidently (1.1)-that concerns only our $B$-is implied by (1.3). By (1.2), there is an equivalence of braided tensor categories $\mathcal{F}:{ }_{L}^{L} \mathcal{Y D} \rightarrow{ }_{K}^{K} \mathcal{Y D}$ with inverse

$$
\mathcal{G}:{ }_{K}^{K} \mathcal{Y D} \rightarrow{ }_{L}^{L} \mathcal{Y} \mathcal{D} \text {. }
$$

Indeed, it is well-known that the Drinfeld doubles of a finite-dimensional Hopf algebra and its dual are isomorphic, so that ${ }_{L}^{L} \mathcal{Y D} \simeq{ }_{B}^{B} \mathcal{Y D}$. Since the tensor categories $B^{*}$-mod and $K^{*}$-mod are equivalent by [S] and because of (1.2), ${ }_{B}^{B} \mathcal{Y D} \simeq{ }_{K}^{K} \mathcal{Y D}$ as braided tensor categories. Now, the simple objects in ${ }_{K}^{K} \mathcal{Y} \mathcal{D}$ are of the form $L(\lambda), \lambda \in \operatorname{Irr}_{\mathbb{k} G}^{\mathbb{k} G} \mathcal{Y} \mathcal{D}$, see Proposition 2.9 below. 
Theorem 1.1. Let $L$ be a basic Hopf algebra, $G$ and $V$ as above. Assume that (1.1) and (1.2) hold. Let $\mathcal{Z}=\mathcal{G}\left(L\left(\lambda_{1}\right)\right) \oplus \cdots \oplus \mathcal{G}\left(L\left(\lambda_{t}\right)\right) \in{ }_{L}^{L} \mathcal{Y D}$

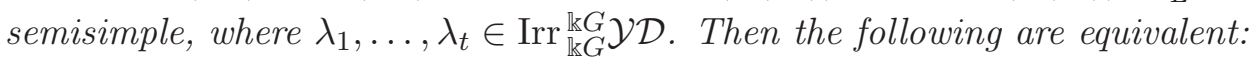

$$
\begin{aligned}
\operatorname{dim} \mathscr{B}(\mathcal{Z}) & <\infty ; \\
\operatorname{dim} \mathscr{B}\left(V \oplus \lambda_{1} \oplus \cdots \oplus \lambda_{t}\right) & <\infty .
\end{aligned}
$$

Since both $\mathcal{F}$ and $\mathcal{G}$ preserve dimensions and Nichols algebras, the proof of Theorem 1.1 is reduced to the following Claim:

Claim A. Let $Z=L\left(\lambda_{1}\right) \oplus \cdots \oplus L\left(\lambda_{t}\right) \in{ }_{K}^{K} \mathcal{Y} \mathcal{D}$, where $\lambda_{1}, \ldots, \lambda_{t} \in \operatorname{Irr}_{\mathbb{k} G}^{\mathbb{k} G} \mathcal{Y} \mathcal{D}$. Then $\operatorname{dim} \mathscr{B}(Z)<\infty$ if and only if (1.5) holds.

Claim A follows from Proposition 2.10, valid for any finite-dimensional Hopf algebra $H$. Observe that the Claim itself does not provide directly new finite-dimensional Hopf algebras as, cf. the proof of Proposition 2.10.

$$
\mathscr{B}(Z) \# K \simeq \mathscr{B}\left(V \oplus \lambda_{1} \oplus \cdots \oplus \lambda_{t}\right) \# \mathbb{k} G .
$$

But the Hopf algebras of the form $\mathscr{B}(\mathcal{Z}) \# L$ are new, except for the small $L$ mentioned above.

Observe that the Nichols algebras $\mathscr{B}(Z)$ bear a Weyl groupoid since $Z$ is semisimple by [AHS, HS2]; these Weyl groupoids were studied in [CL].

Theorem 1.2. Let $L$ be a basic Hopf algebra, $G$ and $V$ as above, such that $G$ is abelian. If $\mathcal{Z} \in{ }_{L}^{L} \mathcal{Y D}$ has $\operatorname{dim} \mathscr{B}(\mathcal{Z})<\infty$, then $\mathcal{Z}$ is semisimple.

This is a drastic simplification, since most of the times ${ }_{L}^{L} \mathcal{Y} \mathcal{D} \simeq{ }_{K}^{K} \mathcal{Y} \mathcal{D}$ is wild. When $G$ is abelian, Theorems 1.1 and 1.2 together with $\mathrm{H} 2$ reduce the complete classification of finite-dimensional Nichols algebras in ${ }_{L}^{L} \mathcal{Y} \mathcal{D}$ to a computational problem. Analogously to the proof of Theorem 1.1, Theorem 1.2 boils down to

Claim B. Assume that $G$ is a finite abelian group. If $Z \in{ }_{K}^{K} \mathcal{Y D}$ has $\operatorname{dim} \mathscr{B}(Z)<\infty$, then $Z$ is semisimple.

Claim B is proved as Theorem 3.9. We point out that Theorems 1.1 and 1.2 generalize, and were motivated by, GG, Theorem 4.5].

1.3. The main result. In Subsection 3.4 we prove that the diagram $R$ of a finite-dimensional Hopf algebra $\mathrm{H}$ whose Hopf coradical is basic is necessarily a Nichols algebra, under suitable hypothesis. Together with the results in $\$ 1.2$, this rounds up the following statement.

Theorem 1.3. Let $L$ be a basic finite-dimensional Hopf algebra such that $G=\operatorname{Hom}_{\text {alg }}(L, \mathbb{k})$ is an abelian group. Let $\mathrm{H}$ be a Hopf algebra with $\mathrm{H}_{[0]} \simeq L$, so that $\mathrm{gr} \mathrm{H} \simeq R \# L$. Then the following are equivalent: 
(a) $\mathrm{H}$ is finite-dimensional, i. e. $R$ is finite-dimensional.

(b) $R \simeq \mathscr{B}(\mathcal{Z})$, where $\mathcal{Z}=\mathcal{G}\left(L\left(\lambda_{1}\right)\right) \oplus \cdots \oplus \mathcal{G}\left(L\left(\lambda_{t}\right)\right) \in{ }_{L}^{L} \mathcal{Y} \mathcal{D}$ is semisimple, with $\lambda_{1}, \ldots, \lambda_{t} \in \operatorname{Irr}_{\mathbb{k}^{k} G}^{\mathbb{k} G} \mathcal{Y D}$, and

$$
\operatorname{dim} \mathscr{B}\left(V \oplus \lambda_{1} \oplus \cdots \oplus \lambda_{t}\right)<\infty .
$$

The assumption $\mathrm{H}_{[0]} \simeq L$ contains implicitly the hypothesis that $L$ is generated by the coradical.

Theorem 1.3 brings down the classification of the finite-dimensional Hopf algebras with Hopf coradical $L$ ( $\operatorname{such}$ that $G=\operatorname{Hom}_{\text {alg }}(L, \mathbb{k})$ is abelian) to two Questions that we formulate in general.

Question 2. For $G$ and $V$ satisfying (1.1) and (1.2), determine

$$
\mathfrak{f}_{G}(V)=\left\{U \in \mathbb{k}_{\mathbb{k} G}^{\mathbb{k} G} \mathcal{Y D}: U \neq 0, \operatorname{dim} \mathscr{B}(U \oplus V)<\infty\right\},
$$

Since ${ }_{\mathbb{k} G}^{\mathbb{k} G} \mathcal{Y} \mathcal{D}$ is semisimple, we have a map from $\mathfrak{f}_{G}(V)$ to the class of semisimple objects in ${ }_{L}^{L} \mathcal{Y} \mathcal{D}$ as above.

Question 3. Classify all liftings of $\mathscr{B}(\mathcal{Z}) \# L$, for any $\mathfrak{f}_{G}(V) \ni U \mapsto \mathcal{Z}$ by the mentioned map.

For given $G$ and $V$, the answer to Question 2 follows from [H2, [HV], up to describing the possible realizations over $G$. Here is an exhaustion result.

Proposition 1.4. Let $L, G, V$ as above. Assume that $L$ is generated by its coradical and that $\mathfrak{f}_{G}(V)=\emptyset$. If $H$ is a finite-dimensional Hopf algebra such that its Hopf coradical $H_{[0]} \simeq L$, then $H \simeq L$.

Indeed, if $\operatorname{dim} \mathscr{B}(\mathcal{Z})<\infty$ for $\mathcal{Z} \in{ }_{L}^{L} \mathcal{Y D}-0$, then $\operatorname{dim} \mathscr{B}(\operatorname{soc} \mathcal{Z})<\infty$.

In otherwords, if $\mathfrak{f}_{G}(V)=\emptyset$, then the only Hopf algebras arising from these circle of ideas are duals of non-trivial liftings of $\mathscr{B}(V) \# \mathbb{k} G$ that are generated by the coradical.

If $G$ is an abelian group of odd order, relatively prime to 105, then there are

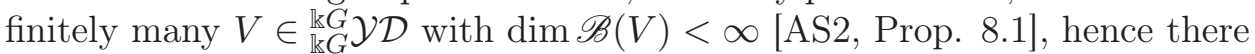
are various $V$ 's with $\mathfrak{f}_{G}(V)=\emptyset$. See also AS2, Theorem 1.3] for $G \simeq \mathbb{Z} / p$. Also, if $G=\mathbb{S}_{3}$, respectively $\mathbb{S}_{5}$, and $V \in \mathbb{k}_{\mathbb{k} G}^{\mathbb{k} G} \mathcal{Y D}$ simple corresponding to the class of transpositions and the sign representation, then $\mathfrak{f}_{G}(V)=\emptyset$ by AHS, $\S 4.2$ ], respectively by [HV].

To implement the usual approach to Question 3 the defining relations of the Nichols algebras $\mathscr{B}\left(L\left(\lambda_{1}\right) \oplus \cdots \oplus L\left(\lambda_{t}\right)\right)$ are needed; these can be computed in principle from those of $\mathscr{B}\left(V \oplus \lambda_{1} \oplus \cdots \oplus \lambda_{t}\right)$. In Section 4 we deal the case of diagonal type; here the existence of a PBW-basis eases up the task. Notice that this is no enough, since the functor $\mathcal{G}$ might not be explicit. Finally in Section 5 we consider decompositions involving a block coming from Nichols algebras in [AAH1]. 
1.4. Decompositions. The proof of Proposition 2.10 relies on a general argument allowing different variations. Let $(W, c)$ be a braided vector space with a decomposition $W=V \oplus U$ such that $V$ and $U$ are braided subspaces and

$$
c(V \otimes U)=U \otimes V, \quad c(U \otimes V)=V \otimes U .
$$

Then $\mathscr{B}(W)$ is not isomorphic to $\mathscr{B}(V) \otimes \mathscr{B}(U)$, unless $c_{\mid V \otimes U} c_{U \otimes V}=\mathrm{id}_{U \otimes V}$ $\mathrm{Gr}$. But there is a substitute, see $\$ 2.3$ for details. Namely, $\mathscr{B}(W)$ splits as

$$
\mathscr{B}(W) \simeq \mathcal{K} \# \mathscr{B}(V)
$$

where $\mathcal{K}$ is an appropriate algebra of coinvariants and \# stands for braided bosonization. By [HS2, Prop. 8.6], $\mathcal{K}$ itself is a Nichols algebra $\mathscr{B}\left(Z_{U}\right)$ where

$$
Z_{U}:=\operatorname{ad}_{c} \mathscr{B}(V)(U) \text {. }
$$

The isomorphism (1.7) is used in the definition of the Weyl groupoid, cf. [H1, AHS, HS2]. In [AAH1, the structure of $\mathscr{B}(W)$ for several families of braided vector spaces $W$ was determined from the knowledge of $\mathcal{K}$ and $\mathscr{B}(V)$ via (1.8). In the present article, we go in the opposite direction and get information on $\mathscr{B}\left(Z_{U}\right)$ from the knowledge of $\mathscr{B}(W)$ and $\mathscr{B}(V)$. At least we get new examples of interesting Nichols algebras, since evidently

$$
\begin{aligned}
\operatorname{dim} \mathscr{B}(W) & =\operatorname{dim} \mathscr{B}\left(Z_{U}\right) \operatorname{dim} \mathscr{B}(V), \\
\mathrm{GK}-\operatorname{dim} \mathscr{B}(W) & \leq \mathrm{GK}-\operatorname{dim} \mathscr{B}\left(Z_{U}\right)+\mathrm{GK}-\operatorname{dim} \mathscr{B}(V),
\end{aligned}
$$

with equality in (1.10) if $\mathscr{B}(V)$ has a convex PBW-basis, cf. AAH1, Lemma 2.3.1 and AAH2, Remark 2.3. Besides, there is some control on $Z_{U}$ : if $V \in{ }_{H}^{H} \mathcal{Y D}, \operatorname{dim} H<\infty, \operatorname{dim} \mathscr{B}(V)<\infty$ and $U \in{ }_{H}^{H} \mathcal{Y D}$ is semisimple, then so is $Z_{U} \in \underset{\mathscr{B}(V) \# H}{\mathscr{B}(V) \# H} \mathcal{Y} \mathcal{D}$, cf. Proposition 2.10, A closely related idea appeared in [R, Section 4] in a different guise and was discussed again in [U3], and as mentioned above in $\mathrm{CL}$.

The paper is organized as follows. In Section 2 Proposition 2.10 is proved. Section 3 is devoted to Nichols algebras of non-semisimple Yetter-Drinfeld modules and the last part of the proof of the main result. We study Nichols algebras arising from decompositions of braided vector spaces of diagonal type in Section 4, respectively with blocks and points in Section 5. There we give partial answers to the following Question, see Theorems 4.4 and 4.5 .

Question 4. A1] Let $\mathscr{B}(V)$ be a Nichols algebra that is a domain and has finite Gelfand-Kirillov dimension. Is $\mathscr{B}(V)$ AS-regular?

Notations. For us, $\mathbb{N}=\{1,2,3, \ldots\}, \mathbb{N}_{0}=\mathbb{N} \cup\{0\}$. If $k<\theta \in \mathbb{N}_{0}$, then we denote $\mathbb{I}_{k, \theta}=\left\{n \in \mathbb{N}_{0}: k \leq n \leq \theta\right\}$, and $\mathbb{I}_{\theta}:=\{1, \ldots, \theta\}$; also $\mathbb{I}=\mathbb{I}_{\theta}$ if $\theta$ is clear from the context. The canonical basis of $\mathbb{Z}^{\theta}$ is denoted by $\left(\alpha_{i}\right)_{i \in \mathbb{I}_{\theta}}$. We set

$$
\alpha_{i j}=\sum_{k \in \mathbb{I}, j} \alpha_{k}, \quad i \leq j \in \mathbb{I}
$$


The group of $n$-th roots of 1 in $\mathbb{k}$ is denoted $\mathbb{G}_{n}, \mathbb{G}_{n}^{\prime}$ is the subset of primitive ones, while $\mathbb{G}_{\infty}=\bigcup_{n \geq 1} \mathbb{G}_{n}, \mathbb{G}_{\infty}^{\prime}=\mathbb{G}_{\infty}-\{1\}$.

Let $\operatorname{Irr} \mathcal{C}$ be the set of isomorphism classes of irreducible objects in an abelian category $\mathcal{C}$. The category of representations of an algebra $A$ is denoted Rep $A$ while the subcategory of finite-dimensional ones is rep $A$. If $M$ is a subobject of $N$ in a category $\mathcal{C}$, then we write $M \leq N$.

See [Mo] for basic results and notations on Hopf algebras. We denote by $\Delta$ the comultiplication of a coalgebra and by $\delta$ the coaction of a comodule. The antipode of a Hopf algebra is denoted by $\mathcal{S}$.

Let $H$ be a Hopf algebra. As usual, $G(H)$ denotes the group of group-like elements in $H$. If $V$ is a (left) $H$-comodule, then $V_{g}:=\{v \in V: \delta(v)=g \otimes v\}$, $g \in G(H)$. The space of skew-primitive elements of a (braided or usual) Hopf algebra $H$ is denoted by $\mathcal{P}_{g, h}(H)$; that of primitive ones simply by $\mathcal{P}(H)$.

\section{Nichols algebras from DeCOMPosable BRAided VeCtor SPACES}

2.1. Preliminaries. We recall the basic definitions and tools to be used along the paper. A pair $(V, c)$ is a braided vector space if $V$ is a vector space and $c \in G L(V \otimes V)$ satisfies

$$
(c \otimes \mathrm{id})(\mathrm{id} \otimes c)(c \otimes \mathrm{id})=(\mathrm{id} \otimes c)(c \otimes \mathrm{id})(\mathrm{id} \otimes c) .
$$

We assume that all Hopf algebras considered here have bijective antipode. Throughout this paper, $H$ is a Hopf algebra. Let ${ }_{H}^{H} \mathcal{Y D}$ be the category of Yetter-Drinfeld modules over $H$ and ${ }_{H}^{H} \mathcal{Y} \mathcal{D}_{\mathrm{fd}}$ the subcategory of finitedimensional ones. Every $V \in{ }_{H}^{H} \mathcal{Y D}$ is a (rigid) braided vector space; recall that the braiding and its inverse are

$$
c(x \otimes y)=x_{(-1)} \cdot y \otimes x_{(0)}, \quad c^{-1}(x \otimes y)=y_{(0)} \otimes \mathcal{S}^{-1}\left(y_{(-1)}\right) \cdot x, \quad x, y \in V .
$$

Conversely every (rigid) braided vector space can be realized in ${ }_{H}^{H} \mathcal{Y} \mathcal{D}$ for a suitable $H$, but by no means in a unique way.

Let $A$ be a Hopf algebra and let $\varkappa: H \otimes A \rightarrow \mathbb{k}$ be a skew-pairing DoT, Definition 1.3], i.e. a linear map such that, for all $a, a^{\prime} \in A$ and $h, h^{\prime} \in H$,

$$
\begin{array}{ll}
\varkappa\left(h, a a^{\prime}\right)=\varkappa\left(h_{(2)}, a\right) \varkappa\left(h_{(1)}, a^{\prime}\right), & \varkappa(h, 1)=\varepsilon(h), \\
\varkappa\left(h h^{\prime}, a\right)=\varkappa\left(h, a_{(1)}\right) \varkappa\left(h^{\prime}, a_{(2)}\right), & \varkappa(1, a)=\varepsilon(a) .
\end{array}
$$

Let $\sigma:(H \otimes A) \otimes(H \otimes A) \rightarrow k$ be the 2-cocycle associated to $\varkappa$, i.e.

$$
\sigma\left(h \otimes a, h^{\prime} \otimes a^{\prime}\right)=\varepsilon(h) \varkappa\left(h^{\prime}, a\right) \varepsilon\left(a^{\prime}\right), \quad a, a^{\prime} \in A, h, h^{\prime} \in H ;
$$

and let $D=(H \otimes A)_{\sigma}$ be the 2-cocycle twist of $H \otimes A$. Namely, $(H \otimes A)_{\sigma}$ is the tensor product coalgebra $H \otimes A$ with multiplication defined by

$$
\begin{aligned}
(h \otimes a)\left(h^{\prime} \otimes a^{\prime}\right) & =\varkappa\left(h_{(1)}^{\prime}, a_{(1)}\right) \varkappa^{-1}\left(h_{(3)}^{\prime}, a_{(3)}\right) h h_{(2)}^{\prime} \otimes a_{(2)} a^{\prime}, \\
\text { where } \varkappa^{-1}(h, a) & =\varkappa(\mathcal{S}(h), a)=\varkappa\left(h, \mathcal{S}^{-1}(a)\right),
\end{aligned}
$$

$a, a^{\prime} \in A, h, h^{\prime} \in H$. The following result essentially goes back to [Dr1]; we include a proof for completeness. 
Lemma 2.1. There is a tensor functor from ${ }_{H}^{H} \mathcal{Y D}$ to $\operatorname{Rep} D$ given by

$$
{ }_{H}^{H} \mathcal{Y D} \ni M \longmapsto M \in \operatorname{Rep} D, \quad(h \otimes a) \cdot m \stackrel{\star}{=} \varkappa\left(m_{(-1)}, a\right) h \cdot m_{(0)},
$$

$a \in A, h \in H, m \in M$.

Proof. Let $a, a^{\prime} \in A, h, h^{\prime} \in H$; set $h=h \otimes a, h^{\prime}=h^{\prime} \otimes a^{\prime}$. Let $m \in M$. Then

$$
\begin{aligned}
& h \cdot\left(h^{\prime} \cdot m\right)=\varkappa\left(m_{(-2)}, a^{\prime}\right) \varkappa\left(h^{\prime}{ }_{(1)} m_{(-1)} \mathcal{S}\left(h^{\prime}{ }_{(3)}\right), a\right)\left(h h^{\prime}(2)\right) \cdot m_{(0)} ; \\
& \left(h h^{\prime}\right) \cdot m=\varkappa\left(h^{\prime}{ }_{(1)}, a_{(1)}\right) \varkappa^{-1}\left(h_{(3)}^{\prime}, a_{(3)}\right) \varkappa\left(m_{(-1)}, a_{(2)} a^{\prime}\right)\left(h h_{(2)}^{\prime}\right) \cdot m_{(0)} \\
& \left.\left.=\varkappa_{\left(h^{\prime}\right.}{ }_{(1)}, a_{(1)}\right) \varkappa\left(\mathcal{S}\left(h_{(3)}^{\prime}\right), a_{(3)}\right) \varkappa\left(m_{(-2)}, a^{\prime}\right) \varkappa_{(-1)}, a_{(2)}\right)\left(h h_{(2)}^{\prime}\right) \cdot m_{(0)}
\end{aligned}
$$

showing that the action $\star$ is associative. By a similar computation, it is compatible with the tensor product.

Assume that $\operatorname{dim} H<\infty$ and take $A=\left(H^{\mathrm{cop}}\right)^{*}, \varkappa$ the standard pairing. Then $D(H)=(H \otimes A)_{\sigma}$ is the Drinfeld double of $H$ and the functor in Lemma 2.1 gives an equivalence of braided tensor categories ${ }_{H}^{H} \mathcal{Y} \mathcal{D}_{\mathrm{fd}} \simeq \operatorname{rep} D(H)$. See [M] or [Mo, 10.6.16].

Let $V \in{ }_{H}^{H} \mathcal{Y D}$. The left dual of $V$ is ${ }^{*} V=\operatorname{Hom}(V, \mathbb{k}) \in{ }_{H}^{H} \mathcal{Y} \mathcal{D}$ with the action and coaction determined by

$$
\langle h \cdot f, x\rangle=\left\langle f, \mathcal{S}^{-1}(h) \cdot x\right\rangle, \quad f_{(-1)}\left\langle f_{(0)}, x\right\rangle=\mathcal{S}\left(x_{(-1)}\right)\left\langle f, x_{(0)}\right\rangle,
$$

$h \in H, x \in V, f \in{ }^{*} V$. We denote by ad, respectively $\operatorname{ad}_{c}$, the adjoint action of a Hopf algebra, respectively of a Hopf algebra in ${ }_{H}^{H} \mathcal{Y} \mathcal{D}$. If $R$ is Hopf algebra in ${ }_{H}^{H} \mathcal{Y} \mathcal{D}$, then the braided commutator of $x, y \in R$ is

$$
[x, y]_{c}=x y-\text { multiplication } \circ c(x \otimes y) .
$$

2.2. Nichols algebras. Nichols algebras bring decisive information for the classification of Hopf algebras (with finite dimension or growth), see AS3, but deserve to be considered as a subject on its own, by their intricate combinatorics and potential relationship with other areas of Algebra. See the survey [A3]. At this stage, we are interested in the following questions:

- Classify all Nichols algebras with finite Gelfand-Kirillov dimension, particularly finite dimension.

- For them, provide a minimal set of defining relations.

There is no hope of a unified approach to these questions, rather one needs first to delimitate classes of braided vector spaces that might be approached uniformly. The class we understand better at this moment is that of braided vector spaces of diagonal type, see below. The class of braided vector spaces over finite groups was treated in many papers, with several substantial answers and lots of intriguing questions, see A3. The class of braided vector spaces over abelian groups but not of diagonal type was considered in AAH1]. We refer to [AS3, AA2, A3 for introductions to Nichols algebras, 
Hopf algebras in braided categories of Yetter-Drinfeld modules, RadfordMajid theory of bosonization, etc. We proceed to recall the definition of Nichols algebra.

Let $V \in{ }_{H}^{H} \mathcal{Y D}$. The tensor algebra $T(V)$ is a Hopf algebra in ${ }_{H}^{H} \mathcal{Y} \mathcal{D}$ (beware, not with the usual comultiplication). Given $f \in{ }^{*} V$, the skewderivation $\partial_{f} \in \operatorname{End} T(V)$ is defined by

$$
\begin{aligned}
\partial_{f}(1) & =0, & \partial_{f}(v)=f(v), \quad v \in V, & \\
\partial_{f}(x y) & =x \partial_{f}(y)+\sum_{j} \partial_{f_{j}}(x) y_{j}, & & \text { where } c^{-1}(y \otimes f)=\sum_{j} f_{j} \otimes y_{j} .
\end{aligned}
$$

Here are some basic properties of the skew-derivations:

- The comultiplication $\Delta: T(V) \rightarrow T(V) \otimes T(V)$ is graded with respect to the standard grading of $T(V)$; let $\Delta^{n-i, i}: T^{n}(V) \rightarrow T^{n-i}(V) \otimes T^{i}(V)$ be its homogeneous component for $n \in \mathbb{N}_{0}$ and $i \in \mathbb{I}_{0, n}$. Then an alternative definition of $\partial_{f}$ is

$$
\partial_{f}=(\mathrm{id} \otimes f) \Delta^{n-1,1}: T^{n}(V) \rightarrow T^{n-1}(V), \quad n \in \mathbb{N} .
$$

○ Let $\mathcal{I}=\oplus_{n \geq 2} \mathcal{I}^{n}$ be a homogeneous Hopf ideal of $T(V)$ and $\mathcal{R}=T(V) / \mathcal{I}$. Then (2.7) defines a skew-derivation $\partial_{f} \in \operatorname{End} \mathcal{R}$, for any $f \in{ }^{*} V$.

o Let $f \in{ }^{*} V, h \in H$ and $x \in T(V)$. It is not difficult to see that

$$
\partial_{f}(h \cdot x)=h_{(1)} \cdot \partial_{\mathcal{S}\left(h_{(2)}\right) \cdot f}(x) .
$$

- Suppose that there exist a basis $\left(x_{i}\right)_{i \in \mathbb{I}}$ of $V$ and a family $\left(g_{i}\right)_{i \in \mathbb{I}}$ in $G(H)$ such that $\delta\left(x_{i}\right)=g_{i} \otimes x_{i}$, for $i \in \mathbb{I}$. Let $\left(f_{i}\right)_{i \in \mathbb{I}}$ be the dual basis to the previous one and $\partial_{i}=\partial_{f_{i}}, i \in \mathbb{I}$. Then to require (2.6) for all $f$ is equivalent to require that for all $i \in \mathbb{I}$

$$
\partial_{i}(x y)=x \partial_{i}(y)+\partial_{i}(x) g_{i} \cdot y, \quad x, y \in T(V) .
$$

Definition 2.2. Let $\mathcal{J}(V)=\oplus_{n \geq 2} \mathcal{J}^{n}(V)$, where for $n \geq 2$,

$$
\mathcal{J}^{n}(V)=\left\{x \in T^{n}(V): \forall f_{1}, \ldots, f_{n} \in{ }^{*} V, \partial_{f_{1}} \ldots \partial_{f_{n}}(x)=0\right\} .
$$

Then $\mathscr{B}(V):=T(V) / \mathcal{J}(V)$ is a Hopf algebra in ${ }_{H}^{H} \mathcal{Y} \mathcal{D}$, called the Nichols algebra of $V$; see e.g. AHS for more details on this approach.

Notice that as an algebra (and coalgebra) $\mathscr{B}(V)$ does depend only on the braiding $c$, by definition of the skew-derivations $\partial_{f}$. As observed, the skew-derivations can be extended to $\mathscr{B}(V)$ and by definition, we have

$$
\bigcap_{f \in \in^{*} V} \operatorname{ker} \partial_{f}=\mathbb{k} 1 \text { in } \mathscr{B}(V) .
$$


2.3. The coinvariant Nichols algebra. We explain how Nichols algebras arise from decomposition, a crucial point in this paper. Let $H$ be a Hopf algebra. Let $V, U \in{ }_{H}^{H} \mathcal{Y D}$ and

$$
W=V \oplus U
$$

Thus we have a decomposition of $W$ as in $\$ 1.4$. Conversely, any decomposition as in $\$ 1.4$ can be realized over a suitable $H$ provided that the braiding of $W$ is rigid.

For simplicity, we set $\mathcal{A}(V)=\mathscr{B}(V) \# H$, etc. The natural morphisms (of Hopf algebras in ${ }_{H}^{H} \mathcal{Y} \mathcal{D}$ ) $\mathscr{B}(W) \rightarrow \mathscr{B}(V)$ and $\mathscr{B}(V) \rightarrow \mathscr{B}(W)$ induce-by tensoring with $\mathrm{id}_{H^{-}}$-morphisms of Hopf algebras

$$
\pi_{\mathcal{A}(V)}: \mathcal{A}(W) \rightarrow \mathcal{A}(V), \quad \text { and } \quad \iota_{\mathcal{A}(V)}: \mathcal{A}(V) \rightarrow \mathcal{A}(W) .
$$

Since $\pi_{\mathcal{A}(V)^{\iota}}{ }_{\mathcal{A}(V)}=\mathrm{id}_{\mathcal{A}(V)}$, the Radford-Majid theory applies. Namely,

$$
\mathcal{K}=\mathcal{A}(W)^{\operatorname{co} \pi_{\mathcal{A}(V)}}
$$

is a Hopf algebra in ${ }_{\mathcal{A}(V)}^{\mathcal{A}(V)} \mathcal{Y} \mathcal{D}$ with the adjoint action and the coaction

$$
\delta=\left(\pi_{\mathcal{A}(V)} \otimes \mathrm{id}\right) \Delta_{\mathcal{A}(W)} .
$$

Remark 2.3. Let $\pi_{H}: \mathcal{A}(V) \rightarrow H$ and $\iota_{H}: H \rightarrow \mathcal{A}(V)$ be the natural projection and inclusion respectively. They give rise to restriction and corestriction functors $\mathcal{A}(V) \mathcal{M} \rightarrow{ }_{H} \mathcal{M}$ and $\mathcal{A}(V) \mathcal{M} \rightarrow{ }^{H} \mathcal{M}$; it is easy to see that they glue together to a tensor functor ${ }_{\mathcal{A}(V)}^{\mathcal{A}(V)} \mathcal{Y} \mathcal{D} \rightarrow{ }_{H}^{H} \mathcal{Y D}$, that however does not preserve braidings.

Furthermore, $\mathcal{A}(W)$ is the biproduct or bosonization of $\mathcal{K}$, that is

$$
\mathcal{A}(W) \simeq \mathcal{K} \# \mathcal{A}(V) .
$$

In fact, $\mathscr{B}(W)$ is the braided bosonization $\mathcal{K} \# \mathscr{B}(V)$, see $\mathrm{AHS}$, HS2 for details. The next result generalizes [R, Proposition 22].

Proposition 2.4. [HS2, Proposition 8.6]. $\mathcal{K} \simeq \mathscr{B}\left(Z_{U}\right)$, where

$$
Z_{U}:=\operatorname{ad}_{c} \mathscr{B}(V)(U) \in_{\mathcal{A}(V)}^{\mathcal{A}(V)} \mathcal{Y D} \text {. }
$$

Of course, interchanging $U$ and $V$, we obtain another Nichols algebra $\mathscr{B}\left(Z_{V}\right)$, where $Z_{V}:=\operatorname{ad}_{c} \mathscr{B}(U)(V) \in_{\mathcal{A}(U)}^{\mathcal{A}(U)} \mathcal{Y D}$.

Remark 2.5. If $c_{V \otimes U} c_{U \otimes V}=\operatorname{id}_{U \otimes V}$, then $Z_{U}=U$ and $\mathcal{K} \simeq \mathscr{B}(U)$.

Notice that $W$ and consequently $\mathscr{B}(W)$ and $\mathcal{K}$ are $\mathbb{Z}^{2}$-graded by

$$
\operatorname{deg} V=\alpha_{1}, \quad \operatorname{deg} U=\alpha_{2},
$$

cf. AHS. Hence every pair of integers $\left(d_{1}, d_{2}\right)$ defines a $\mathbb{Z}$-grading of $\mathscr{B}(W)$ via the group homomorphism $\mathbb{Z}^{2} \rightarrow \mathbb{Z}, \alpha_{i} \mapsto d_{i}$. The usual $\mathbb{Z}$-grading of $\mathscr{B}(W)$ comes from the pair $(1,1)$. Also, the grading of $\mathcal{K}$ as a Nichols algebra arises from the given by $(0,1)$, i.e. $\operatorname{deg} U=1, \operatorname{deg} V=0$; thus $\operatorname{deg} Z_{U}=1$. 
Consider the grading from the pair $(1,0)$. Then $Z_{U}=\oplus_{j \geq 0} Z_{U}^{j}$ is $\mathbb{Z}$-graded, with

$$
Z_{U}^{0}=U, \quad Z_{U}^{j}=\operatorname{ad}_{c} \mathscr{B}^{j}(V)(U) \leq \mathscr{B}^{j+1}(W) \text { in }{ }_{H}^{H} \mathcal{Y} \mathcal{D} .
$$

Lemma 2.6. Let $N \leq Z_{U}$ in $\underset{\mathcal{A}(V)}{\mathcal{A}(V)} \mathcal{Y} \mathcal{D}$. If $N=\oplus_{j \geq 1}\left(N \cap Z_{U}^{j}\right)$, then $N=0$.

Proof. Let $\mathcal{I}$ be the 2 -sided ideal of $\mathscr{B}(W)$ generated by $N$. We claim that $\mathcal{I}$ is a Hopf ideal. Indeed, let $\varpi_{\mathscr{B}(W)}: \mathcal{A}(W) \rightarrow \mathscr{B}(W)$ be the usual projection. Write $\delta(n)=n_{(-1)} \otimes n_{(0)} \in \mathcal{A}(V) \otimes N$ and compute

$$
\begin{aligned}
& \Delta_{\mathcal{A}(W)}(n)=n \otimes 1+n_{(-1)} \otimes n_{(0)} \Longrightarrow \\
& \Delta_{\mathscr{B}(W)}(n)=n \otimes 1+\varpi_{\mathscr{B}(W)}\left(n_{(-1)}\right) \otimes n_{(0)} \in N \otimes \mathscr{B}(W)+\mathscr{B}(W) \otimes N,
\end{aligned}
$$

since $N$ is a subcomodule of $Z_{U}$. Now, if $M$ is a subcomodule of $Z_{U}$, then

$$
c(M \otimes \mathscr{B}(W))=\mathscr{B}(W) \otimes M, \quad c(\mathscr{B}(W) \otimes M)=M \otimes \mathscr{B}(W),
$$

braiding in ${ }_{H}^{H} \mathcal{Y} \mathcal{D}$, since the action and the coaction of $H$ on $M$ are the restriction of the action of $\mathcal{A}(V)$, respectively the corestriction of the coaction of $\mathcal{A}(V)$. The claim follows by a recursive argument. Since $N$ is positively graded by hypothesis, $\mathcal{I} \subseteq \oplus_{n \geq 2} \mathscr{B}^{n}(W)$. Thus $\mathcal{I}$, and a fortiori $N$, is 0 .

We next characterize $\mathscr{B}\left(Z_{U}\right)$ using the skew-derivations $\partial_{f}$. Clearly, we have a decomposition ${ }^{*} W \simeq{ }^{*} V \oplus^{*} U$ in ${ }_{H}^{H} \mathcal{Y} \mathcal{D}$, thus we have skew-derivations $\partial_{f}$ of $\mathscr{B}(W)$ for all $f \in{ }^{*} V$, extending those of $\mathscr{B}(V)$.

Proposition 2.7. $\mathscr{B}\left(Z_{U}\right)=\bigcap_{f \in{ }^{*} V} \operatorname{ker} \partial_{f}$.

Proof. We first claim that $\mathbf{K}:=\bigcap_{f \in^{*} V}$ ker $\partial_{f}$ is a subalgebra of $\mathscr{B}(W)$; this follows from (2.6) since $c^{-1}\left(\mathscr{B}(W) \otimes{ }^{*} V\right)={ }^{*} V \otimes \mathscr{B}(W)$. Also, $\mathbf{K}$ is stable under the action of $H$ by (2.8). We next claim that $\operatorname{ad}_{c}(V)(\mathbf{K}) \subseteq \mathbf{K}$. For, let $f \in{ }^{*} V, x \in V$ and $y \in \mathbf{K}$. Then

$$
\begin{aligned}
\partial_{f}\left(\operatorname{ad}_{c}(x) y\right) & =\partial_{f}\left(x y-\left(x_{(-1)} \cdot y\right) x_{(0)}\right)=x \partial_{f}(y)+\left\langle f_{(0)}, x\right\rangle \mathcal{S}^{-1}\left(f_{(1)}\right) \cdot y \\
& -\left(x_{(-1)} \cdot y\right)\left\langle f, x_{(0)}\right\rangle-\partial_{f_{(0)}}\left(x_{(-1)} \cdot y\right) \mathcal{S}^{-1}\left(f_{(1)}\right) \cdot x_{(0)}=0 .
\end{aligned}
$$

Now $U \subset \mathbf{K}$, hence $Z_{U}=\operatorname{ad}_{c} \mathscr{B}(V)(U) \subset \mathbf{K}$, thus $\mathscr{B}\left(Z_{U}\right) \subseteq \mathbf{K}$, since $\mathscr{B}\left(Z_{U}\right)$ is generated by $Z_{U}$ as algebra.

Conversely, let $x \in \mathbf{K}$. Fix a basis $\left(k_{s}\right)_{s \in S}$ of $\mathscr{B}\left(Z_{U}\right)$. As the multiplication induces a linear isomorphism $\mathscr{B}(W) \simeq \mathscr{B}\left(Z_{U}\right) \# \mathscr{B}(V)$, we have

$$
x=\sum_{s \in S} k_{s} a_{s}, \quad \text { for some } a_{s} \in \mathscr{B}(V) .
$$

Given $f \in{ }^{*} V$, we have that $0=\partial_{f}(x)=\sum_{s \in S} k_{s} \partial_{f}\left(a_{s}\right)$ since $k_{s} \in \operatorname{ker} \partial_{f}$, thus $\partial_{f}\left(a_{s}\right)=0$, hence $a_{s} \in \mathbb{k}$ for all $s \in S$ by (2.11). Thus $x \in \mathscr{B}\left(Z_{U}\right)$. 
2.4. Nichols algebras of semisimple Yetter-Drinfeld modules. Let $H$ be a Hopf algebra with bijective antipode, $V \in{ }_{H}^{H} \mathcal{Y D}$ and $\mathcal{A}(V)=\mathscr{B}(V) \# H$ as before. We first recall the construction of a family of irreducible YetterDrinfeld modules of $\mathcal{A}(V)$ from [AHS, §3.3], cf. also [R]. Given $\lambda \in \operatorname{Irr}_{H}^{H} \mathcal{Y} \mathcal{D}$, take $W=V \oplus \lambda \in{ }_{H}^{H} \mathcal{Y D}$, so that $\mathscr{B}(W)$ has a decomposition $\mathscr{B}(W)=$ $\mathcal{K} \# \mathscr{B}(V)$. Then $L(\lambda):=\operatorname{ad}_{c} \mathscr{B}(V)(\lambda) \subset \mathcal{K}$ belongs to ${ }_{\mathcal{A}(V)}^{\mathcal{A}(V)} \mathcal{Y} \mathcal{D}$.

Proposition 2.8. AHS, Lemma 3.3 \& Proposition 3.5] The module $L(\lambda)$ is simple and the map $L: \operatorname{Irr}{ }_{H}^{H} \mathcal{Y D} \rightarrow \operatorname{Irr}_{\mathcal{A}(V)}^{\mathcal{A}(V)} \mathcal{Y D}, \lambda \mapsto L(\lambda)$, is injective. Also $L(\lambda)=\oplus_{n \in \mathbb{N}} L(\lambda)^{n}$ is a graded Yetter-Drinfeld submodule of $\mathcal{P}(\mathcal{K})$ over $\mathcal{A}(V)$ (and over $H$ ) with $L(\lambda)^{1}=\lambda$.

Under some conditions on $H$ and $V$ the family of simple Yetter-Drinfeld modules $L(\lambda)$ exhaust those of ${ }_{\mathcal{A}(V)}^{\mathcal{A}(V)} \mathcal{Y} \mathcal{D}$ or of a closely related category. For simplicity of the exposition, and because of our main goals in this paper, we shall consider the following assumptions:

$$
\begin{aligned}
& H \text { is a finite-dimensional Hopf algebra. } \\
& V \in{ }_{H}^{H} \mathcal{Y D} \text { has } \operatorname{dim} \mathscr{B}(V)<\infty .
\end{aligned}
$$

If both assumptions hold, then $\mathcal{A}(V)$ has finite dimension and its dual is isomorphic to $\mathscr{B}(\bar{V}) \# H^{*}$, where $\bar{V}=\operatorname{Hom}(V, \mathbb{k}) \in{ }_{H^{*}}^{H^{*}} \mathcal{Y} \mathcal{D}$ in such a way that for $v \in V, x \in \bar{V}, h \in H, a \in H^{*}$,

$$
(x \mid h \cdot v)=\left(x_{(-1)} \mid h\right)\left(x_{(0)} \mid v\right), \quad(a \cdot x \mid v)=\left(a \mid \mathcal{S}\left(v_{(-1)}\right)\right)\left(x \mid v_{(0)}\right) .
$$

Let $\mathfrak{D}=D(\mathscr{B}(V) \# H)$ be the Drinfeld double of $\mathscr{B}(V) \# H$. The multiplication induces a triangular decomposition:

$$
\mathfrak{D} \simeq \mathscr{B}(V) \otimes D(H) \otimes \mathscr{B}(\bar{V}) .
$$

Now the assignment $\operatorname{deg} V=1=-\operatorname{deg} \bar{V}, \operatorname{deg} D(H)=0$, induces a grading on $\mathfrak{D}$; notice that this is opposite to the grading in $[\mathrm{PV}]$. Accordingly,

$$
\mathfrak{D}^{\leq 0}:=\mathscr{B}(\bar{V}) \# D(H) \simeq D(H) \otimes \mathscr{B}(\bar{V}) \hookrightarrow \mathfrak{D} \hookleftarrow \mathfrak{D}^{\geq 0}:=\mathscr{B}(V) \# D(H) .
$$

Every $D(H)$-module becomes a $\mathfrak{D} \leq 0$-module with the trivial action of $\mathscr{B}(\bar{V})$. Let $\lambda \in \operatorname{Irr} \operatorname{Rep} D(H) \simeq \operatorname{Irr}_{H}^{H} \mathcal{Y} \mathcal{D}$. We consider the Verma module

$$
M(\lambda)=\operatorname{Ind}_{\mathfrak{D} \leq 0}^{\mathfrak{D}} \lambda=\mathfrak{D} \otimes_{\mathfrak{D} \leq 0} \lambda \simeq \mathscr{B}(V) \otimes \lambda .
$$

Notice that $M(\lambda)=\oplus_{n \geq 0} M^{n}(\lambda)$ is a graded $\mathfrak{D}$-module, where $M^{n}(\lambda)=$ $\mathscr{B}^{n}(V) \otimes \lambda$ up to the isomorphism above. Thus $M^{0}(\lambda) \simeq \lambda$ in ${ }_{H}^{H} \mathcal{Y} \mathcal{D}$. Set $M_{+}(\lambda)=\oplus_{n>0} M^{n}(\lambda)$.

The following result is known for $H=\mathbb{k} G$ a group algebra; it was proved in [HY1 when $G$ abelian and in PV] for any finite $G$, although the main ideas of the proof appeared much earlier [L, RS]. Here we only assume (2.14) and (2.15). We consider the simple modules $L(\lambda)$ of Proposition 2.8 with the grading shifted in -1 ; that is, $L(\lambda)=\oplus_{n \geq 0} L(\lambda)^{n}$, where $L(\lambda)^{0}=\lambda$. 
Proposition 2.9. The Verma module $M(\lambda)$ has a unique simple quotient, which is graded and isomorphic to $L(\lambda)$. The family $L(\lambda), \lambda \in \operatorname{Irr}_{H}^{H} \mathcal{Y D}$, is a parametrization of Irr Rep $\mathfrak{D}$.

Proof. We start by the uniqueness; cf. the proofs of [PV, Theorems $1 \& 3]$.

Claim 1. Let $x \in M^{0}(\lambda) \simeq \lambda, x \neq 0$ and $y \in M_{+}(\lambda)$. Then $M(\lambda)=$ $\mathfrak{D}^{\geq 0}(x+y)$.

Indeed, let $N=\mathfrak{D}^{\geq 0}(x+y)$. If $y=0$, then $D(H) \cdot x=M^{0}(\lambda) \hookrightarrow N$, since $\lambda$ is simple, and $\mathfrak{D}^{\geq 0} \cdot x=\mathscr{B}(V) \cdot M^{0}(\lambda)=N$. Assume that $y \neq 0$ and write $y=y_{1}+y_{2}$, where $0 \neq y_{1} \in M^{h}(\lambda)$ and $y_{2} \in \oplus_{j>h} M^{j}(\lambda)$. By the preceding, there exists $z \in \mathscr{B}^{h}(V) \# D(H)$ such that $z \cdot x=y_{1}$. Then

$$
x+y-z \cdot(x+y)=x+y_{2}-z \cdot y \in N, \quad \text { and } \quad y_{2}-z \cdot y \in \oplus_{j>h} M^{j}(\lambda) .
$$

Arguing recursively, we may assume that $h=$ top but then $y_{2}-z \cdot y=0$.

Claim 2. $M(\lambda)$ has a unique maximal submodule $N(\lambda)$, which is graded.

Indeed, let $N(\lambda)$ be the sum of all graded submodules contained in $M_{+}(\lambda)$. Let $N \leq M(\lambda)$ and let $\widetilde{N}$ be the span of all homogeneous components of all elements in $N$; clearly $\widetilde{N}$ is also a submodule of $M(\lambda)$. Now $N \neq M(\lambda)$ implies $\tilde{N} \subseteq M_{+}(\lambda)$ by Claim 1. Thus any proper submodule of $M(\lambda)$ is contained in $N(\lambda)$, as expected.

Thus we have a unique simple quotient $M(\lambda) / N(\lambda)$, which is graded. Let us see that it is isomorphic to $L(\lambda)$. By Lemma 2.1 we may consider $L(\lambda)$ as a (graded) $\mathfrak{D}$-module. Hence $\bar{V}$ acts trivially on $L(\lambda)^{0}=\lambda$, by looking at the degree. Hence there exists a map $\pi: M(\lambda) \rightarrow L(\lambda)$ of $\mathfrak{D}$-modules; as $L(\lambda)$ is generated by $\mathscr{B}(V)$ as $\mathscr{B}(V) \# H$-module, $\pi$ is surjective. Since $L(\lambda)$ is simple by Proposition 2.8, we have $L(\lambda) \simeq M(\lambda) / N(\lambda)$.

Finally, let $L$ be a finite-dimensional $\mathfrak{D}$-module and let $S$ be a simple $\mathfrak{D} \geq 0$. submodule of $L$. Necessarily $S \simeq \lambda$ for some $\lambda \in \operatorname{Irr}_{H}^{H} \mathcal{Y D}$ with trivial action of $\mathscr{B}(V)$. Thus we have a non-zero morphism of $\mathcal{D}$-modules $M(\lambda) \rightarrow L$; if $L$ is simple, then this is an epimorphism and thus $L \simeq L(\lambda)$.

We have identified the simple modules from Proposition 2.8 as quotients of Verma modules, but we can also derive Proposition 2.8 from Lemma 2.6. Say for this purpose that $L(\lambda)$ is the unique simple quotient of $M(\lambda)$.

Proposition 2.10. Let $\lambda_{1}, \ldots \lambda_{t} \in \operatorname{Irr}_{H}^{H} \mathcal{Y} \mathcal{D}$. Then

$$
\mathscr{B}\left(V \oplus \lambda_{1} \oplus \cdots \oplus \lambda_{t}\right) \simeq \mathscr{B}\left(L\left(\lambda_{1}\right) \oplus \cdots \oplus L\left(\lambda_{t}\right)\right) \# \mathscr{B}(V) .
$$


Proof. In the context of Proposition 2.4 take $U=\lambda_{1} \oplus \cdots \oplus \lambda_{t}$. We have a projection $\pi: M\left(\lambda_{1}\right) \oplus \cdots \oplus M\left(\lambda_{t}\right) \rightarrow Z_{\lambda_{1} \oplus \cdots \oplus \lambda_{t}}$, homogeneous with respect to the grading considered in Lemma 2.6, i. e. corresponding to $(1,0)$. Let $N=\pi\left(N\left(\lambda_{1}\right) \oplus \cdots \oplus N\left(\lambda_{t}\right)\right)$; clearly $N \subset \oplus_{j>0} Z_{U}^{j}$. By Lemma 2.6, we conclude that $N=0$.

In this way, Propositions 2.9 and 2.10 reduce the calculation of Nichols algebras of semisimple Yetter-Drinfeld modules over $\mathcal{A}(V)$ to the knowledge of Nichols algebras of semisimple Yetter-Drinfeld modules over $H$. As an illustration, we work out the example considered in GG].

Example 2.11. Let $\Gamma \simeq \mathbb{Z} / N$ where $1<N \in \mathbb{N}$. Fix $g \in \Gamma$ a generator, so that $\Gamma=\left\{g^{a}: a \in \mathbb{Z} / N\right\}$; fix $q \in \mathbb{G}_{N}^{\prime}$ and let $\eta \in \widehat{\Gamma}$ given by $\eta(g)=q$, so that $\widehat{\Gamma}=\left\{\eta^{b}: b \in \mathbb{Z} / N\right\}$. Let $\mathbb{k}_{a}^{b}=\mathbb{k}_{g^{a}}^{\eta^{b}} \in{ }_{\mathbb{k} \Gamma}^{\mathbb{k} \Gamma} \mathcal{Y} \mathcal{D}$ be the one-dimensional object with action given by $\eta^{b}$ and coaction by $g^{a}$. If $a, b, c, d \in \mathbb{Z} / N$, then $\mathbb{k}_{a}^{b} \oplus \mathbb{K}_{c}^{d}$ is of diagonal type with Dynkin diagram $\stackrel{q^{a b}}{\circ} \underline{q^{a d+b c}} q^{c d}$.

Assume that $N=4$ and let $V=\mathbb{k}_{1}^{2}$, so that $\mathscr{B}(V)=\Lambda(V)$ has dimension 2. Let $\Theta=\Theta_{0} \cup \Theta_{1} \cup \Theta_{3}$, where

$$
\Theta_{0}=\{(1,2),(3,2)\}, \Theta_{1}=\{(1,3),(2,1),(3,3)\}, \Theta_{3}=\{(1,1),(2,3),(3,1)\} .
$$

If $(a, b) \in \mathbb{Z} / N \times \mathbb{Z} / N-\Theta$, then $\operatorname{dim} \mathscr{B}\left(\mathbb{k}_{a}^{b}\right)=\infty$. The following claims are proved by inspection in the classification list [H2].

Claim 1. The following are equivalent:

(1) $\operatorname{dim} \mathscr{B}\left(V \oplus \mathbb{k}_{a}^{b}\right)<\infty$.

(2) $(a, b) \in \Theta-\{(3,1),(3,3)\}$.

If this is the case, then:

- For $(a, b) \in \Theta_{0}$, we have $\operatorname{dim} \mathscr{B}\left(Z_{\mathbb{R}_{a}^{b}}\right)=2$. Indeed the Dynkin diagram of $V \oplus \mathbb{k}_{a}^{b}$ is in both cases $\begin{array}{cc}-1 & -1 \\ 0 & 0\end{array}$.

- For $(a, b) \in\{(1,3),(2,1),(1,1),(2,3)\}$, we have $\operatorname{dim} \mathscr{B}\left(Z_{\mathbb{k}_{a}^{b}}\right)=8$. Indeed the Dynkin diagrams of $V \oplus \mathbb{k}_{a}^{b}$ are respectively

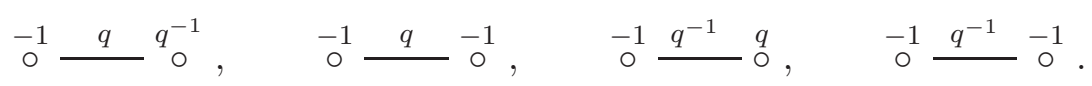

These are of type $\mathbf{A}(1 \mid 1)$, see $\underline{\mathrm{AA2}}$, §5.1.11], and $\operatorname{dim} \mathscr{B}\left(V \oplus \mathbb{k}_{a}^{b}\right)=16$.

Claim 2. Let $\left(a_{i}, b_{i}\right) \in \Theta_{0}, i \in \mathbb{I}_{M}$, and $U=\oplus_{i \in \mathbb{I}_{M}} \mathbb{k}_{a_{i}}^{b_{i}}$. Then $Z_{U}=U$ and $\mathscr{B}(U)$ has dimension $2^{M}$. Indeed the Dynkin diagram of $V \oplus U$ consists of disconnected points. 
Claim 3. Let $(a, b),(c, d) \in \Theta-\{(3,1),(3,3)\}$ with $(a, b) \notin \Theta_{0}$, and $U=$ $\mathbb{k}_{a}^{b} \oplus \mathbb{k}_{c}^{d}$. Then the following are equivalent:

(1) $\operatorname{dim} \mathscr{B}(V \oplus U)<\infty$.

(2) One of the next possibilities occurs:

(a) $U=\mathbb{k}_{1}^{3} \oplus \mathbb{k}_{1}^{2}$; the Dynkin diagram of $V \oplus U$ is $\stackrel{-1}{\circ} \stackrel{q}{q^{-1}} \stackrel{q}{\circ} \stackrel{-1}{\circ}$.

(b) $U=\mathbb{k}_{1}^{3} \oplus \mathbb{k}_{1}^{1}$; the Dynkin diagram of $\mathbb{k}_{1}^{3} \oplus V \oplus \mathbb{k}_{1}^{1}$ is $\stackrel{q^{-1}}{\circ} \stackrel{q}{-} \stackrel{-1}{\circ} \stackrel{q^{-1}}{\longrightarrow} \stackrel{q}{\circ}$.

(c) $U=\mathbb{k}_{2}^{1} \oplus \mathbb{k}_{3}^{2}$; the Dynkin diagram of $V \oplus U$ is $\stackrel{-1}{\circ} \stackrel{q}{\circ} \stackrel{-1}{\circ} \stackrel{q^{-1}}{-} \stackrel{-1}{\circ}$.

(d) $U=\mathbb{k}_{2}^{1} \oplus \mathbb{k}_{2}^{3}$; the Dynkin diagram of $\mathbb{k}_{2}^{1} \oplus V \oplus \mathbb{k}_{2}^{3}$ is $\stackrel{-1}{\circ} \stackrel{q}{\circ} \stackrel{-1}{\circ} \stackrel{q^{-1}}{\circ} \stackrel{-1}{\circ}$.

(e) $U=\mathbb{k}_{1}^{1} \oplus \mathbb{k}_{1}^{2}$; the Dynkin diagram of $V \oplus U$ is $\stackrel{-1}{\circ} \stackrel{q^{-1}}{\longrightarrow} \stackrel{q}{\circ} \stackrel{q^{-1}}{-1} \stackrel{-1}{\circ}$.

(f) $U=\mathbb{k}_{2}^{3} \oplus \mathbb{k}_{3}^{2}$; the Dynkin diagram of $V \oplus U$ is $\stackrel{-1}{\circ} \stackrel{q^{-1}}{-} \stackrel{-1}{\circ} \stackrel{q}{\longrightarrow}{ }^{-1}$.

Now $\operatorname{dim} \mathscr{B}(V \oplus U)=256$ because all these Dynkin diagrams are of type $\mathbf{A}(2 \mid 1)$ [AA2, §5.1.8]. Thus $\operatorname{dim} \mathscr{B}\left(Z_{U}\right)=128$ in all cases.

Claim 4. Let $U \in \mathbb{k}_{\mathbb{k} \Gamma}^{\mathbb{k} \Gamma \mathcal{Y}}$ of dimension 3. Then either $\operatorname{dim} \mathscr{B}\left(Z_{U}\right)=\infty$ or all simple submodules of $U$ have labels in $\Theta_{0}$.

Indeed, $U=\mathbb{k}_{a}^{b} \oplus \mathbb{k}_{c}^{d} \oplus \mathbb{k}_{e}^{f}$, where $(a, b),(c, d),(e, f) \in \Theta-\{(3,1),(3,3)\}$. We may assume that $(a, b) \notin \Theta_{0}$. Then $\mathbb{k}_{a}^{b} \oplus \mathbb{k}_{c}^{d}$ and $\mathbb{k}_{a}^{b} \oplus \mathbb{k}_{e}^{f}$ belong to the list in Claim 3 reducing drastically the possibilities. Then we proceed by inspection again. Collecting together these Claims, we have:

Proposition 2.12. Let $K=\mathscr{B}(V) \# \mathbb{k} \Gamma$, where $\Gamma$ is cyclic of order 4 and $V=\mathbb{k}_{1}^{2}$ as above. Let $Z=\oplus_{i \in \mathbb{I}_{M}} L\left(\mathbb{k}_{a_{i}}^{b_{i}}\right) \in{ }_{K}^{K} \mathcal{Y D}$. Then the following are equivalent:

(1) $\operatorname{dim} \mathscr{B}(Z)<\infty$.

(2) One of the next possibilities occurs:

(a) All $L\left(\mathbb{k}_{a_{i}}^{b_{i}}\right)$ have dimension 1; then $\operatorname{dim} \mathscr{B}(Z)=2^{M}$.

(b) $M=1$ and $\mathbb{k}_{a}^{b}$ is as in Claim 1 . There are 4 examples, all with $\operatorname{dim} \mathscr{B}(Z)=8$.

(c) $M=2$ and $\mathbb{k}_{a_{1}}^{b_{1}} \oplus \mathbb{k}_{a_{2}}^{b_{2}}$ is as in Claim 3. There are 6 examples, all with $\operatorname{dim} \mathscr{B}(Z)=128$. 
Let $K$ be as in Proposition 2.12, let $L$ be the Radford Hopf algebra of dimension 8 and let $\mathcal{G}:{ }_{K}^{K} \mathcal{Y D} \rightarrow{ }_{L}^{L} \mathcal{Y D}$ be the equivalence of braided tensor categories as in the Introduction. The classification of the finite-dimensional Nichols algebras $\mathscr{B}(Z)$ with $Z \in{ }_{L}^{L} \mathcal{Y D}$ was addressed in GG. If $\operatorname{dim} \mathscr{B}(Z)<$ $\infty$, then $Z$ should be semisimple by [GG, Theorem 4.5] and the classification is achieved assuming that $Z$ is simple [GG, Theorem A]. The classification might be concluded as an application of the previous result.

Corollary 2.13. Let $Z \in{ }_{L}^{L} \mathcal{Y D}$. The following are equivalent:

(1) $\operatorname{dim} \mathscr{B}(Z)<\infty$.

(2) $Z$ is semisimple and one of the next possibilities occurs:

(a) All simple submodules of $Z$ have dimension 1; then $\operatorname{dim} \mathscr{B}(Z)=2^{\operatorname{dim} Z}$.

(b) $Z$ is one of the simple objects $\mathcal{G}\left(L\left(\mathbb{k}_{a}^{b}\right)\right)$, where $\mathbb{k}_{a}^{b}$ is as in Claim 1 . There are 4 examples, all with $\operatorname{dim} \mathscr{B}(Z)=8$.

(c) $Z$ is one of $\mathcal{G}\left(L\left(\mathbb{k}_{a_{1}}^{b_{1}}\right)\right) \oplus \mathcal{G}\left(L\left(\mathbb{k}_{a_{2}}^{b_{2}}\right)\right)$ where $\mathbb{k}_{a_{1}}^{b_{1}} \oplus \mathbb{k}_{a_{2}}^{b_{2}}$ is as in Claim 3. There are 6 examples, all with $\operatorname{dim} \mathscr{B}(Z)=128$.

To be precise one needs to identify the simple objects $\mathcal{G}\left(L\left(\mathbb{k}_{a}^{b}\right)\right)$.

\section{Semisimplicity And the Diagram}

Here we prove the second and third parts of the proof of our main Theorem 1.3 .

3.1. Nichols algebras of graded or filtered Yetter-Drinfeld modules. We start by some generalities mostly well-known.

3.1.1. Graded. Let $\mathcal{A}=\oplus_{i \in \mathbb{Z}} \mathcal{A}^{i}$ be a graded Hopf algebra, i.e. $\mathcal{A}^{i} \mathcal{A}^{j} \subseteq \mathcal{A}^{i+j}$ and $\Delta\left(\mathcal{A}^{i}\right) \subseteq \sum_{h+k=i} \mathcal{A}^{h} \otimes \mathcal{A}^{k}$ for all $i, j \in \mathbb{Z}$ (then the antipode preserves the grading).

A graded Yetter-Drinfeld module over $\mathcal{A}$ is $M \in{ }_{\mathcal{A}}^{\mathcal{A}} \mathcal{Y} \mathcal{D}$ provided with a grading $M=\oplus_{j \in \mathbb{Z}} M^{j}$ such that

$$
\mathcal{A}^{i} \cdot M^{j} \subseteq M^{i+j}, \quad \delta\left(M^{j}\right) \subseteq \sum_{h+k=j} \mathcal{A}^{h} \otimes M^{k}, \quad i, j \in \mathbb{Z} .
$$

The category gr- ${ }_{\mathcal{A}}^{\mathcal{A}} \mathcal{Y} \mathcal{D}$ of graded Yetter-Drinfeld modules over $\mathcal{A}$, with maps preserving all structures, is a braided tensor category: the unit object $\mathbb{k}$ has degree 0 ; if $M, N \in \operatorname{gr}-{ }_{\mathcal{A}}^{\mathcal{A}} \mathcal{Y} \mathcal{D}$, then $M \otimes N \in \operatorname{gr}-{ }_{\mathcal{A}}^{\mathcal{A}} \mathcal{Y D}$ with the grading $(M \otimes N)^{j}=\sum_{h+k=j} M^{h} \otimes N^{k}$, and the braiding $c_{M, N}$ is homogeneous.

For instance, if $\mathcal{A}=\oplus_{i \in \mathbb{Z}} \mathcal{A}^{i}$ is finite dimensional, then $\mathcal{A}^{*}=\oplus_{i \in \mathbb{Z}}\left(\mathcal{A}^{*}\right)^{i}$ is also a graded Hopf algebra, where $\left(\mathcal{A}^{*}\right)^{i}=\left(\mathcal{A}^{-i}\right)^{*}$, up to natural identifications. Then $D(\mathcal{A})$ is also a graded Hopf algebra, and gr- ${ }_{\mathcal{A}}^{\mathcal{Y}} \mathcal{Y} \mathcal{D}$ is equivalent, as braided tensor category, to that of graded $D(\mathcal{A})$-modules. 
Remark 3.1. Let $\mathcal{A}=\oplus_{i \in \mathbb{N}_{0}} \mathcal{A}^{i}$ be a graded Hopf algebra. Then the coradical $\mathcal{A}_{0}$ coincides with the coradical of $\mathcal{A}^{0}$. In particular, if $\mathcal{A}^{0}$ is pointed, then so is $\mathcal{A}$ and $G(\mathcal{A})=G\left(\mathcal{A}^{0}\right)$.

Proof. The family $\mathcal{F}_{n} \mathcal{A}=\oplus_{i \in \mathbb{I}_{0, n}} \mathcal{A}^{i}$ is a coalgebra filtration of $\mathcal{A}$. By [Mo, Lemma 5.3.4], $\mathcal{A}_{0} \subseteq \mathcal{F}_{0} \mathcal{A}=\mathcal{A}^{0}$, so $\mathcal{A}_{0} \subseteq\left(\mathcal{A}^{0}\right)_{0} \subseteq \mathcal{A}_{0}$.

If $M \in \operatorname{gr}-{ }_{A}^{\mathcal{A}} \mathcal{Y} \mathcal{D}$, then $T(M)$ is a Hopf algebra in $\operatorname{gr}-{ }_{A}^{\mathcal{A}} \mathcal{Y} \mathcal{D}$, and so is $\mathscr{B}(M)$ (because the quantum symmetrizer is homogeneous). We consider the $\mathbb{Z}^{2}$-gradings on these algebras given by

$$
\operatorname{deg} T^{n}(M)^{j}=\operatorname{deg} \mathscr{B}^{n}(M)^{j}=(j, n), \quad j \in \mathbb{Z}, n \in \mathbb{N}_{0} .
$$

Then $T(M)$ and $\mathscr{B}(M)$ are $\mathbb{Z}^{2}$-graded algebras and coalgebras, but beware they are not $\mathbb{Z}^{2}$-graded algebras in ${ }_{\mathcal{A}}^{\mathcal{A}} \mathcal{Y} \mathcal{D}$ as $T^{n}(M)^{j}$ is not a Yetter-Drinfeld submodule. However we have:

Lemma 3.2. The bosonizations $T(M) \# \mathcal{A}$ and $\mathscr{B}(M) \# \mathcal{A}$ are $\mathbb{Z}^{2}$-graded Hopf algebras, with grading given by

$$
\operatorname{deg} \mathscr{B}^{n}(M)^{j} \# \mathcal{A}^{i}=(i+j, n), \quad i, j \in \mathbb{Z}, n \in \mathbb{N}_{0} .
$$

Proof. This is straightforward:

$$
\begin{aligned}
& \left(\mathscr{B}^{n}(M)^{j} \# \mathcal{A}^{i}\right)\left(\mathscr{B}^{m}(M)^{r} \# \mathcal{A}^{s}\right) \subseteq \sum_{h+k=i} \mathscr{B}^{n}(M)^{j} \mathcal{A}^{h} \cdot \mathscr{B}^{m}(M)^{r} \# \mathcal{A}^{k} \mathcal{A}^{s} \\
& \subseteq \sum_{h+k=i} \mathscr{B}^{n}(M)^{j} \mathscr{B}^{m}(M)^{r+h} \# \mathcal{A}^{k+s} \subseteq \sum_{h+k=i} \mathscr{B}^{n+m}(M)^{j+r+h} \# \mathcal{A}^{k+s} ; \\
& \Delta\left(\mathscr{B}^{n}(M)^{j} \# \mathcal{A}^{i}\right) \subseteq \sum_{\substack{p+q=n \\
h+k=i \\
r+s+t=j}} \mathscr{B}^{p}(M)^{r} \# \mathcal{A}^{s} \mathcal{A}^{h} \otimes \mathscr{B}^{q}(M)^{t} \# \mathcal{A}^{k} .
\end{aligned}
$$

3.1.2. Filtered. As above, let $\mathcal{A}=\oplus_{i \in \mathbb{Z}} \mathcal{A}^{i}$ be a graded Hopf algebra. A filtered Yetter-Drinfeld module over $\mathcal{A}$ is $M \in{ }_{\mathcal{A}}^{\mathcal{A}} \mathcal{Y D}$ provided with an ascending filtration $\left(M_{(j)}\right)_{j \in \mathbb{Z}}$ such that $M=\bigcup_{j \in \mathbb{Z}} M_{(j)}$ and

$$
\mathcal{A}^{i} \cdot M_{(j)} \subseteq M_{(i+j)}, \quad \delta\left(M_{(j)}\right) \subseteq \sum_{h+k=j} \mathcal{A}^{h} \otimes M_{(k)}, \quad i, j \in \mathbb{Z} .
$$

The category filt- ${ }_{\mathcal{A}}^{\mathcal{Y}} \mathcal{Y} \mathcal{D}$ of filtered Yetter-Drinfeld modules over $\mathcal{A}$, with maps preserving all structures, is a braided tensor category: if $M, N \in$ filt- ${ }_{\mathcal{A}}^{\mathcal{Y}} \mathcal{D}$, then $M \otimes N \in$ filt- ${ }_{\mathcal{A}}^{\mathcal{Y}} \mathcal{Y} \mathcal{D}$ with filtration

$$
(M \otimes N)_{(j)}=\sum_{h+k=j} M_{(h)} \otimes N_{(k)},
$$

so that the braiding $c_{M, N}$ preserves the filtration. 
Remark 3.3. (a) There is a forgetful functor gr- ${ }_{\mathcal{A}}^{\mathcal{A}} \mathcal{Y} \mathcal{D} \rightarrow$ filt- ${ }_{\mathcal{A}}^{\mathcal{A}} \mathcal{Y} \mathcal{D}:$

$$
M=\oplus_{k \in \mathbb{Z}} M^{k} \in \text { gr- }{ }_{\mathcal{A}}^{\mathcal{A}} \mathcal{Y D} \rightsquigarrow \quad\left(M_{(j)}\right)_{j \in \mathbb{Z}}, \quad M_{(j)}=\oplus_{k \leq j} M^{k}, \quad j \in \mathbb{Z} .
$$

(b) If $\pi: M \rightarrow N$, is a projection in ${ }_{\mathcal{A}}^{\mathcal{A}} \mathcal{Y} \mathcal{D}$ and $M=\bigcup_{j \in \mathbb{Z}} M_{(j)}$ is filtered, then $N$ is also filtered: $N=\bigcup_{j \in \mathbb{Z}} N_{(j)}, N_{(j)}:=\pi\left(M_{(j)}\right)$.

(c) As a consequence, every quotient of a Verma module $M(\lambda)$ as in Proposition 2.9 is a filtered Yetter-Drinfeld module.

If $M \in$ filt- ${ }_{\mathcal{A}}^{\mathcal{A}} \mathcal{Y} \mathcal{D}$, then $T^{n}(M)=M^{\otimes n} \in$ filt- ${ }_{\mathcal{A}}^{\mathcal{A}} \mathcal{Y} \mathcal{D}$ by (3.1), and also $\mathscr{B}^{n}(M) \in$ filt- ${ }_{\mathcal{A}}^{\mathcal{A}} \mathcal{Y} \mathcal{D}$ by Remark 3.3 (b). By (3.1) again,

$$
T^{m}(M)_{(i)} T^{n}(M)_{(j)} \subseteq T^{m+n}(M)_{(i+j)},
$$

and since the braiding preserves the filtration:

$$
\Delta\left(T^{m}(M)_{(i)}\right) \subseteq \sum_{\substack{n+p=m \\ j+k=i}} T^{n}(M)_{(j)} \otimes T^{p}(M)_{(k)} .
$$

Thus the corresponding properties hold in $\mathscr{B}(V)$ by Remark 3.3 (b)

$$
\begin{aligned}
\mathscr{B}^{m}(M)_{(i)} \mathscr{B}^{n}(M)_{(j)} & \subseteq \mathscr{B}^{m+n}(M)_{(i+j)}, \\
\Delta\left(\mathscr{B}^{m}(M)_{(i)}\right) & \subseteq \sum_{\substack{n+p=m \\
j+k=i}} \mathscr{B}^{n}(M)_{(j)} \otimes \mathscr{B}^{p}(M)_{(k)} .
\end{aligned}
$$

Lemma 3.4. The Hopf algebra $\mathscr{B}(M) \# \mathcal{A}$ is filtered, with filtration

$$
(\mathscr{B}(M) \# \mathcal{A})_{(n)}=\sum_{i+j+k \leq n} \mathscr{B}^{i}(M)_{(j)} \# \mathcal{A}^{k}, \quad n \in \mathbb{Z} .
$$

Proof. By (3.2) the filtration (3.3) is of algebras and coalgebras:

$$
\begin{aligned}
& \left(\mathscr{B}^{n}(M)_{(j)} \# \mathcal{A}^{i}\right)\left(\mathscr{B}^{m}(M)_{(r)} \# \mathcal{A}^{s}\right) \subseteq \sum_{h+k=i} \mathscr{B}^{n}(M)_{(j)} \mathcal{A}^{h} \cdot \mathscr{B}^{m}(M)_{(r)} \# \mathcal{A}^{k} \mathcal{A}^{s} \\
& \subseteq \sum_{h+k=i} \mathscr{B}^{n}(M)_{(j)} \mathscr{B}^{m}(M)_{(r+h)} \# \mathcal{A}^{k+s} \subseteq \sum_{h+k=i} \mathscr{B}^{n+m}(M)_{(j+r+h)} \# \mathcal{A}^{k+s} \\
& \Delta\left(\mathscr{B}^{n}(M)_{(j)} \# \mathcal{A}^{i}\right) \subseteq \sum_{\begin{array}{c}
p+q=n \\
h+k=i \\
r+s+t=j
\end{array}} \mathscr{B}^{p}(M)_{(r)} \# \mathcal{A}^{s} \mathcal{A}^{h} \otimes \mathscr{B}^{q}(M)_{(t)} \# \mathcal{A}^{k} .
\end{aligned}
$$

Finally the antipode preserves the filtration since

$$
\begin{aligned}
\mathcal{S}\left(\mathscr{B}^{n}(M)_{(j)} \# \mathcal{A}^{i}\right) & \subseteq \sum_{h+k=j}\left(1 \# \mathcal{S}_{h}\left(\mathcal{A}^{h} \mathcal{A}^{i}\right)\right)\left(\mathcal{S}_{\mathscr{B}(M)}\left(\mathscr{B}^{n}(M)_{(k)}\right) \# 1\right) \\
& \subseteq \sum_{h+k=j}\left(1 \# \mathcal{A}^{h+i}\right)\left(\mathscr{B}^{n}(M)_{(k)} \# 1\right) \subseteq(\mathscr{B}(M) \# \mathcal{A})_{(n+j+i)} .
\end{aligned}
$$

That is, (3.3) is a Hopf algebra filtration. 
Remark 3.5. The statement holds also for pre-Nichols algebras of $M$, i.e. graded connected Hopf algebras in ${ }_{\mathcal{A}(V)}^{\mathcal{A}(V)} \mathcal{Y} \mathcal{D}$ generated by the degree one component which is isomorphic to $M$.

3.2. Pointed Hopf algebras. We state two facts needed later in the proof of the main result.

Let $B$ be a finite-dimensional pointed Hopf algebra with $G=G(B)$ abelian. The graded Hopf algebra gr $B=\oplus_{n \geq 0} \operatorname{gr}^{n} B$ associated to the coradical filtration of $B$ splits as gr $B \simeq R \# \mathbb{k} G ; R=\oplus_{n \geq 0} R^{n}$ is a graded Hopf algebra in $\underset{\mathbb{K} G}{\mathbb{R} G} \mathcal{Y} \mathcal{D}$ and $\mathbf{V}=R^{1}$ is the infinitesimal braiding of $B$. Now the projection $B_{1} \rightarrow B_{1} / B_{0}$ admits a section $\mathfrak{s}: B_{1} / B_{0} \rightarrow B_{1}$ of $\mathbb{k} G$-bimodules. In fact

$$
B_{1}=\mathbb{k} G \oplus \mathfrak{s}(\mathbf{V}) \mathbb{k} G .
$$

Let $\theta:=\operatorname{dim} \mathbf{V}, \mathbb{I}=\mathbb{I}_{\theta}$. We fix a basis $\left(x_{i}\right)_{i \in \mathbb{I}}$ of $\mathbf{V}$ such that the $G$-action and the $G$-coaction on $x_{i}$ are given by $\chi_{i} \in \widehat{G}$ and $g_{i} \in G$, respectively, for all $i \in \mathbb{I}$. This choice of the basis induces $\mathbb{Z}^{\mathbb{I}}$-gradings on $T(\mathbf{V})$ and $\mathscr{B}(\mathbf{V})$. If $r \in T(\mathbf{V})$ is $\mathbb{Z}^{\mathbb{I}}$-homogeneous of degree $\left(a_{i}\right)_{i \in \mathbb{I}}$, then we set $\chi_{r}=\prod_{i \in \mathbb{I}} \chi_{i}^{a_{i}}$. Quite a bit is known about the structure of $B$ :

○ The list of all $\mathbf{V}$ with $\operatorname{dim} \mathscr{B}(\mathbf{V})<\infty$ is known by [H2].

- By An2 $R \simeq \mathscr{B}(\mathbf{V})$ as mentioned in the Introduction.

$\circ$ In An1, An2 there was described a minimal set of homogeneous relations $\mathfrak{G} \subset T(\mathbf{V})$ defining $\mathscr{B}(\mathbf{V})$.

○ By [AnG, Theorem 1.1] (as a culmination of a series of papers with other authors), there exists a family $\boldsymbol{\lambda}=\left(\lambda_{r}\right)_{r \in \mathfrak{G}}$ in $\mathbb{k}^{\mathfrak{G}}$ with the restriction $\lambda_{r}=0$ if $\chi_{r} \neq \epsilon$, such that $K \simeq \mathfrak{u}(\boldsymbol{\lambda})$; here $\mathfrak{u}(\boldsymbol{\lambda})$ is presented as the quotient $T(\mathbf{V}) \# \mathbb{k} G$ by relations

$$
r-p_{r}(\boldsymbol{\lambda}), \quad r \in \mathfrak{G}
$$

where $p_{r}(\boldsymbol{\lambda}) \in \oplus_{k<\operatorname{deg} r} T^{k}(\mathbf{V}) \# \mathbb{k} G$ are defined recursively.

We shall use a refinement of these facts to prove the next result; we were unable to find a simpler proof.

Lemma 3.6. Let $B$ be a finite-dimensional pointed Hopf algebra with $G=$ $G(B)$ abelian; keep the notation above. Let $\mathbf{V}^{\prime} \leq \mathbf{V}$ in $\mathbb{k}_{\mathbb{k} G}^{\mathbb{k} G \mathcal{D}}$ and let $B^{\prime}$ be the subalgebra of $B$ generated by $\mathfrak{s}\left(\mathbf{V}^{\prime}\right)$ and $G$. Then $B^{\prime}$ is a lifting of $\mathscr{B}\left(\mathbf{V}^{\prime}\right) \# \mathbb{k} G$, particularly

$$
\operatorname{dim} B^{\prime}=\operatorname{dim} \mathscr{B}\left(\mathbf{V}^{\prime}\right)|G| .
$$

Proof. Evidently $B^{\prime}$ is a Hopf subalgebra of $B$, in particular it is pointed. Since $\mathbf{V}^{\prime} \hookrightarrow$ the infinitesimal braiding of $B^{\prime}$, we have

$$
\operatorname{dim} B^{\prime} \geq \operatorname{dim} \mathscr{B}\left(\mathbf{V}^{\prime}\right)|G| .
$$

Let $t:=\operatorname{dim} \mathbf{V}^{\prime} \leq \theta$. By AnG2, Lemma 3.7], there exist 
- a basis $\left(x_{i}\right)_{i \in \mathbb{I}}$ of $\mathbf{V}$ as described above, such that $\left(x_{i}\right)_{i \in \mathbb{I}_{t}}$ is a basis of $\mathbf{V}^{\prime}$;

- a minimal set of homogeneous (with respect to the basis above) relations

$\mathfrak{G} \subset T(\mathbf{V})$ defining $\mathscr{B}(\mathbf{V})$, such that $\mathfrak{G}^{\prime}=\mathfrak{G} \cap T\left(V^{\prime}\right)$, (i.e. the relations with only letters $\left.\left(x_{i}\right)_{i \in \mathbb{I}_{t}}\right)$ is a minimal set of relations defining $\mathscr{B}\left(V^{\prime}\right)$;

$\circ$ and such that if $\boldsymbol{\lambda}=\left(\lambda_{r}\right)_{r \in \mathfrak{G}}$ is a family in $\mathbb{k}^{\mathfrak{G}}$ as above such that $B \simeq \mathfrak{u}(\boldsymbol{\lambda})$, then $\boldsymbol{\lambda}^{\prime}:=\left(\lambda_{r}\right)_{r \in \mathfrak{G}^{\prime}}$ satisfies

$$
p_{r}(\boldsymbol{\lambda})=p_{r}\left(\boldsymbol{\lambda}^{\prime}\right), \quad r \in \mathfrak{G}^{\prime}
$$

and $\mathfrak{u}\left(\boldsymbol{\lambda}^{\prime}\right)=T\left(\mathbf{V}^{\prime}\right) \# \mathbb{k} G /\left\langle r-p_{r}\left(\boldsymbol{\lambda}^{\prime}\right): r \in \mathfrak{G}^{\prime}\right\rangle$ is a a lifting of $\mathscr{B}\left(\mathbf{V}^{\prime}\right) \# \mathbb{k} G$.

Since clearly there is a surjective map $\mathfrak{u}\left(\boldsymbol{\lambda}^{\prime}\right) \rightarrow B^{\prime}$, we have

$$
\operatorname{dim} B^{\prime} \leq \operatorname{dim} \mathfrak{u}\left(\boldsymbol{\lambda}^{\prime}\right)=\operatorname{dim} \mathscr{B}\left(\mathbf{V}^{\prime}\right)|G|
$$

that together with (3.6) gives (3.5).

Lemma 3.7. Let $T=\oplus_{n \geq 0} T^{n}$ be a finite-dimensional graded connected Hopf algebra in $\mathbb{k}_{\mathbb{k} G} \mathcal{Y} \mathcal{D}$ and $B=T \# \mathbb{k} G$, so that $B$ is pointed. Then there is a monomorphism $\mathcal{P}(T) \hookrightarrow \mathbf{V}$, the infinitesimal braiding of $B$.

Proof. Clearly there is a monomorphism $\mathcal{P}(T) \hookrightarrow B_{1}$; then apply (3.4).

3.3. Semisimplicity. Again we assume that $H$ and $V$ satisfy (2.14) and (2.15). We consider the following question:

Question 5. Let $Z \in{ }_{H}^{H} \mathcal{Y D}$. Does the finiteness of $\operatorname{dim} \mathscr{B}(Z)$ imply that $Z$ is semisimple?

Remark 3.8. If $Z$ provides a negative answer to Question 5, then we may assume that $Z / S$ is simple, where $S$ is the socle of $Z$.

Indeed, let $L$ be a simple subobject of $Z / S$ and consider $\widetilde{Z}=p^{-1}(L) \subset Z$, where $p: Z \rightarrow Z / S$ is the projection. Then the socle of $\widetilde{Z}$ is again $S$, $\mathscr{B}(\widetilde{Z}) \subset \mathscr{B}(Z)$ is finite-dimensional and $\widetilde{Z} / S \simeq L$, so we replace $Z$ by $\widetilde{Z}$.

We answer affirmatively Question 5 when $H$ is the group algebra of a finite abelian group.

Theorem 3.9. Let $H=\mathbb{k} G$, where $G$ is a finite abelian group, and let $V$ satisfy (2.15). Set $\mathcal{A}(V)=\mathscr{B}(V) \# \mathbb{k} G$. Let $Z \in_{\mathcal{A}(V)}^{\mathcal{A}(V)} \mathcal{Y D}$. If $\operatorname{dim} \mathscr{B}(Z)<\infty$, then $Z$ is semisimple.

We start by a general Lemma.

Lemma 3.10. Let $H$ be an arbitrary Hopf algebra, $R$ a Hopf algebra in ${ }_{H}^{H} \mathcal{Y D}$ and $\mathcal{H}=R \# H$. Then $\mathcal{P}_{g, 1}(\mathcal{H}) \subset \mathcal{P}(R) \# 1+1 \# \mathcal{P}_{g, 1}(H)$ for all $g \in G$. 
Proof. We fix a basis $\left(x_{i}\right)_{i \in I}$ of $R$ such that for a distinguished $i_{0} \in I, x_{i_{0}}=1$ and $\epsilon\left(x_{i}\right)=0$ when $i \neq i_{0}$. Let $w=\sum_{i \in I} x_{i} \# k_{i} \in \mathcal{P}_{g, 1}(\mathcal{H})$, where $k_{i} \in H$, $i \in I$. We apply id \# id $\otimes \epsilon \#$ id to the equality

$$
\Delta(w)=\sum_{i \in I}\left(x_{i}\right)^{(1)} \#\left(x_{i}\right)_{(-1)}^{(2)}\left(k_{i}\right)_{(1)} \otimes\left(x_{i}\right)_{(0)}^{(2)} \#\left(k_{i}\right)_{(2)}=w \otimes 1 \# 1+1 \# g \otimes w
$$

and get

$$
\sum_{i \in I} x_{i} \#\left(k_{i}\right)_{(1)} \otimes\left(k_{i}\right)_{(2)}=1 \# k_{i_{0}} \otimes 1+\sum_{i_{0} \neq i \in I} x_{i} \# k_{i} \otimes 1+1 \# g \otimes k_{i_{0}},
$$

hence

$$
\Delta\left(k_{i}\right)=k_{i} \otimes 1, i \in I, i \neq i_{0} ; \quad \Delta\left(k_{i_{0}}\right)=k_{i_{0}} \otimes 1+g \otimes k_{i_{0}} .
$$

That is, $k_{i} \in \mathbb{k}$ for $i \neq i_{0} ; k_{i_{0}} \in \mathcal{P}_{g, 1}(H) \subset \mathcal{P}_{g, 1}(\mathcal{H})$ and $w=z \# 1+1 \# k_{i_{0}}$, where $z=\sum_{i_{0} \neq i \in I} x_{i} k_{i}$. Then $z \# 1 \in \mathcal{P}_{g, 1}(\mathcal{H})$. We apply id $\# \epsilon \otimes$ id $\#$ id to the equality

$$
\Delta(z \# 1)=(z)^{(1)} \#(z)_{(-1)}^{(2)} \otimes(z)_{(0)}^{(2)} \# 1=z \# 1 \otimes 1 \# 1+1 \# g \otimes z \# 1
$$

and get

$$
\Delta(z)=(z)^{(1)} \otimes(z)^{(2)}=z \otimes 1+1 \otimes z
$$

Thus $z \in \mathcal{P}(R)$.

Let $H$ and $V$ be as in (2.14) and (2.15). Then $\mathcal{A}(V)=\oplus_{i \geq 0} \mathcal{A}^{i}(V)$ is a graded Hopf algebra where $\mathcal{A}^{i}(V)=\mathscr{B}^{i}(V) \# H$. Here is the key step.

Lemma 3.11. Assume that $H=\mathbb{k} G$, where $G$ is a finite abelian group. Let $N \subseteq M_{+}(\lambda)$ be a submodule of $M(\lambda)$ and let $M=M(\lambda) / N \in{ }_{\mathcal{A}(V)}^{\mathcal{A}(V)} \mathcal{Y D}$. If $\operatorname{dim} \mathscr{B}(M)<\infty$, then $M=L(\lambda)$.

Thus the braided bosonization of a Nichols algebra does not need to be a Nichols algebra. Compare with [U3, Thm. 4.3.1].

Proof. Let $J=\mathscr{B}(M) \# \mathcal{A}(V)$. The filtration of $M(\lambda)$ arising from its grading induces a filtration of $M$, and thus a Hopf algebra filtration on $J$. By (3.3), the first two terms of this filtration are

$$
J_{(0)}=\mathbb{k} \# \mathbb{k} \# \mathbb{k} G \simeq \mathbb{k} G, \quad J_{(1)}=\mathbb{k} \# \mathbb{k} \# \mathbb{k} G \oplus \mathbb{k} \# V \# \mathbb{k} G \oplus \lambda \# \mathbb{k} \# \mathbb{k} G .
$$

Hence $J_{0}=\mathbb{k} G$ by $\left[\mathrm{Mo}\right.$, Lemma 5.3.4] and $J$ is pointed. Let $\mathbf{V} \in \mathbb{R}_{\mathbb{k} G}^{\mathbb{Y} G \mathcal{D}}$ be the infinitesimal braiding of $J$; by (3.8) there is a monomorphism

$$
\mathbf{V}^{\prime}:=V \oplus \lambda \hookrightarrow \mathbf{V} .
$$


Let $J^{\prime}$ be the subalgebra of $J$ generated by $\mathbf{V}^{\prime}$ and $G$, that is by $J_{1}$. We claim that $J^{\prime}=J$. Indeed $J$ is generated by $M, V, G$ but $M=\operatorname{ad} \mathscr{B}(V) \cdot \lambda$, implying the claim. By Lemma 3.6 and the last claim,

$$
\operatorname{dim} \mathscr{B}\left(\mathbf{V}^{\prime}\right)|G|=\operatorname{dim} J^{\prime}=\operatorname{dim} J=\operatorname{dim} \mathscr{B}(M) \operatorname{dim} \mathscr{B}(V)|G| .
$$

By Proposition 2.10, $\operatorname{dim} \mathscr{B}\left(\mathbf{V}^{\prime}\right)=\operatorname{dim} \mathscr{B}(V) \operatorname{dim} \mathscr{B}(L(\lambda))$, implying that $\operatorname{dim} \mathscr{B}(L(\lambda))=\operatorname{dim} \mathscr{B}(M)$. As $M$ projects onto $L(\lambda)$, we conclude that $M=L(\lambda)$.

We are ready to complete the proof.

Proof of Theorem 3.9. We fix some notation:

○ $S$ is the socle of $Z, S=L\left(\lambda_{1}\right) \oplus \cdots \oplus L\left(\lambda_{t}\right)$ for some $\lambda_{i} \in \operatorname{Irr}_{\mathbb{k} G}^{\mathbb{k} G} \mathcal{Y} \mathcal{D}$.

○ $\mathcal{H}:=\mathscr{B}(Z) \# \mathcal{A}(V), \mathcal{H}^{\prime}:=\mathscr{B}(S) \# \mathcal{A}(V)$. We may consider $\mathcal{H}^{\prime}$ as a Hopf subalgebra of $\mathcal{H}$, since $\mathscr{B}(S)$ is a Hopf subalgebra of $\mathscr{B}(Z)$ in ${ }_{\mathcal{A}(V)}^{\mathcal{A}(V)} \mathcal{Y} \mathcal{D}$.

By Remark 3.1, $\mathcal{H}$ is pointed and $G(\mathcal{H})=G$. Thus $\mathcal{H}$ is generated by $\mathcal{H}_{0}=\mathbb{k} G$ and the skew-primitive elements. Now we want to describe $\mathcal{P}_{g, 1}(\mathcal{H})$ for each $g \in G$ : By Lemma 3.10, with $R=\mathscr{B}(Z)$,

$$
\mathcal{P}_{g, 1}(\mathcal{H}) \subset Z \# 1+1 \# \mathcal{P}_{g, 1}(H), \quad g \in G .
$$

We next determine the elements of $Z$ that are skew-primitive in $\mathcal{H}$. Set

$$
Z^{\prime}=\sum_{g \in G} Z_{g}^{\prime}, \quad \text { where } Z_{g}^{\prime}:=\left\{z \in Z \mid z \# 1 \in \mathcal{P}_{g, 1}(\mathcal{H})\right\}, g \in G .
$$

We check that

$$
h \cdot z \in Z_{h g h^{-1}}^{\prime}=Z_{g}^{\prime}, \quad \delta_{h} \cdot z=\delta_{g, h} z, \quad \text { for all } g, h \in G, z \in Z_{g}^{\prime} .
$$

Hence $Z^{\prime}$ is a $D(\mathbb{k} G)$-submodule of $Z$. Moreover, $Z^{\prime}=\oplus_{g \in G} Z_{g}^{\prime}$.

Step 1. $F \cdot z=0$ for all $F \in \bar{V}$ and all $z \in Z^{\prime}$.

Let $\left(E_{k}\right)_{k \in \mathbb{I}_{M}}$ be a basis of $\mathscr{B}(V)$, where $E_{1}=1$, each $E_{k}$ is homogeneous with respect to the $\mathbb{N}_{0}$-grading and the $\mathbb{k} G$-coaction is given by $g_{k} \in G$. Let $\left(F_{k}\right)_{k \in \mathbb{I}_{M}}$ be the dual basis of $\mathscr{B}(\bar{V})$. Let $z \in Z_{g}^{\prime}, g \in G$. Hence,

$$
\begin{aligned}
\Delta(z \# 1) & \stackrel{\star}{=} z \# 1 \otimes 1 \# 1+1 \# g \otimes z \# 1 \\
& \stackrel{*}{=} z \# 1 \otimes 1 \# 1+\sum_{k \in \mathbb{I}_{M}} 1 \# E_{k} g g_{k}^{-1} \otimes F_{k} \cdot z \# 1 ;
\end{aligned}
$$

here $\star$ is merely by assumption, while $*$ comes from the formulas for the bosonization. Thus the Step follows.

Step 2. $Z^{\prime} \subset S$. 
We decompose $Z^{\prime}=\oplus_{i \in \mathbb{I}_{t}} \mu_{i}$, each $\mu_{i}$ a simple $D(\mathbb{k} G)$-module. Let $z \in \mu_{i}$, $z \neq 0, i \in \mathbb{I}_{t}$. By the previous Step, $F \cdot z=0$ for all $F \in \bar{V}$. Thus, there exists a surjective map $M\left(\mu_{i}\right) \rightarrow \mathfrak{D} \cdot z=\mathfrak{D} \geq 0 \cdot z$. Also, $\mathscr{B}(\mathfrak{D} \cdot z) \subseteq \mathscr{B}(Z)$, so $\operatorname{dim} \mathscr{B}(\mathfrak{D} \cdot z)<\infty$. By Lemma $3.11, \mathfrak{D} \cdot z \simeq L\left(\mu_{i}\right)$, so $z \in S$.

Step 3. The subalgebra generated by $\mathcal{H}_{0} \cup\left(\cup_{g \in G} \mathcal{P}_{g, 1}(\mathcal{H})\right)$ is $\mathcal{H}^{\prime}$.

From the previous steps, the skew-primitive elements of $\mathcal{H}$ belong either to $S \# 1$ or else to $1 \# \mathcal{A}(V)$. Notice that

$$
\mathcal{H}^{\prime}=\mathscr{B}(S) \# \mathcal{A}(V)=\mathscr{B}(S) \#(\mathscr{B}(V) \# \mathbb{k} G) \simeq \mathscr{B}\left(V \oplus \lambda_{1} \oplus \cdots \oplus \lambda_{t}\right) \# \mathbb{k} G .
$$

Thus the subalgebra generated by $\mathbb{k} G$ and the skew-primitive elements is $\mathcal{H}^{\prime}$.

By the last Step, $\mathcal{H}^{\prime}=\mathcal{H}$. As $\mathcal{H}^{\prime} \cap Z \# 1=S \# 1$, we have that $S=Z$.

3.4. The diagram. We consider the following setting:

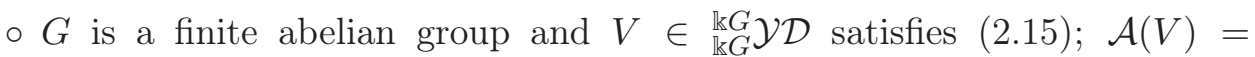
$\mathscr{B}(V) \# \mathbb{k} G=\oplus_{i \in \mathbb{N}_{0}} \mathscr{B}^{i}(V) \# \mathbb{k} G$.

$\circ \mathrm{R}=\oplus_{i \in \mathbb{N}_{0}} \mathrm{R}^{i}$ is a finite-dimensional connected graded Hopf algebra in ${ }_{\mathcal{A}(V)}^{\mathcal{A}(V)} \mathcal{Y} \mathcal{D}$, generated by $Z:=\mathrm{R}^{1}$. Thus we have an epimorphism $\mathrm{R} \rightarrow \mathscr{B}(Z)$ of Hopf algebras in ${ }_{\mathcal{A}(V)}^{\mathcal{H}(V)} \mathcal{Y} \mathcal{D}$. In other words, $\mathrm{R}$ is a pre-Nichols algebra of $Z$.

Proposition 3.12. $\mathrm{R} \simeq \mathscr{B}(Z)$.

Proof. By Theorem 3.9, $Z$ is semisimple, say $Z=L\left(\lambda_{1}\right) \oplus \cdots \oplus L\left(\lambda_{t}\right)$, where

$\lambda_{1}, \ldots, \lambda_{t} \in \operatorname{Irr}_{\mathbb{k} G}^{\mathbb{R} G} \mathcal{Y D}$. Particularly, $Z$ is a graded object of ${ }_{\mathcal{A}(V)}^{\mathcal{A}(V)} \mathcal{Y} \mathcal{D}$, hence so is $T(Z)$, and consequently $\mathrm{R}$ is a filtered Hopf algebra in ${ }_{K}^{K} \mathcal{Y} \mathcal{D}$, and $a$ fortiori $J:=\mathrm{R} \# \mathcal{A}(V)$ is a filtered Hopf algebra, with filtration given by

$$
J_{(n)}=\sum_{i+j+k \leq n} \mathrm{R}_{(j)}^{i} \# \mathscr{B}^{k}(V) \# \mathbb{k} G, \quad n \in \mathbb{N}_{0},
$$

by (the same proof as) Lemma 3.4, cf. Remark 3.5. Now the proof follows as the one of Lemma 3.11. Indeed $\mathrm{R} \# \mathcal{A}(V)$ is pointed by [Mo, Lemma 5.3.4], with coradical $\mathbb{k} G$. Let $\mathbf{V}$ be the infinitesimal braiding of $J$. Then

$$
\mathbf{V}^{\prime}:=V \oplus \lambda_{1} \oplus \cdots \oplus \lambda_{t} \hookrightarrow \mathbf{V}
$$

Let $J^{\prime}$ be the subalgebra of $J$ generated by $\mathbf{V}^{\prime}$ and $G$. We claim that $J^{\prime}=J$. Indeed $J$ is generated by $Z, V, G$ but $Z=\operatorname{ad} \mathscr{B}(V) \cdot\left(\lambda_{1} \oplus \cdots \oplus \lambda_{t}\right)$, implying the claim. By Lemma 3.6 and the last claim,

$$
\operatorname{dim} \mathscr{B}\left(\mathbf{V}^{\prime}\right)|G|=\operatorname{dim} J^{\prime}=\operatorname{dim} J=\operatorname{dim} R \operatorname{dim} \mathscr{B}(V)|G| .
$$

By Proposition 2.10, $\operatorname{dim} \mathscr{B}\left(\mathbf{V}^{\prime}\right)=\operatorname{dim} \mathscr{B}(V) \operatorname{dim} \mathscr{B}(Z)$, so $\mathrm{R} \simeq \mathscr{B}(Z)$.

We need one more general result before proving the main Theorem. 
Lemma 3.13. Let $\mathrm{H}$ be a Hopf algebra with Hopf coradical $\mathrm{H}_{[0]} \simeq H$, so that gr $\mathrm{H} \simeq R \# H$ for some connected graded Hopf algebra $R=\oplus_{i \in \mathbb{N}_{0}} R^{i} \in{ }_{H}^{H} \mathcal{Y D}$. Then $\mathcal{P}(R)=R^{1}$.

Proof. As $R$ is connected and graded, $R^{1} \subseteq \mathcal{P}(R)$. On the other hand, let $x \in \mathcal{P}(R)$. By [AC, Proposition 1.2],

$$
(\operatorname{gr~H})_{[0]}=R^{0} \# H \simeq H, \quad(\operatorname{gr~H})_{[1]}=R^{0} \# H \oplus R^{1} \# H .
$$

By direct computation,

$$
\begin{aligned}
\Delta(x \# 1) & =x \# 1 \otimes 1 \# 1+1 \# x_{(-1)} \otimes x_{(0)} \# 1 \\
& \in \operatorname{gr~H} \otimes(\operatorname{gr~H})_{[0]}+(\operatorname{gr~H})_{[0]} \otimes \operatorname{gr~H} .
\end{aligned}
$$

Hence $x \# 1 \in(\operatorname{gr~H})_{[0]} \wedge(\text { gr H })_{[0]}=(\operatorname{gr~H})_{[1]}$, so $x \in R^{1}$.

If $H$ is cosemisimple, then it follows from the previous result that $R$ is coradically graded AS1. But we do not know whether this is true in general.

Proof of Theorem 1.3. Recall that $L$ is a basic finite-dimensional Hopf algebra such that $G=\operatorname{Hom}_{\text {alg }}(L, \mathbb{k})$ is abelian and $\mathrm{H}$ is a Hopf algebra with $\mathrm{H}_{[0]} \simeq L$, so that gr $\mathrm{H} \simeq R \# L$. Also $B=L^{*}$ and $K=\operatorname{gr} B=\mathscr{B}(V) \# \mathbb{k} G$. Since (1.2) holds, we have inverse equivalences of braided tensor categories $\mathcal{F}:{ }_{L}^{L} \mathcal{Y D} \rightarrow{ }_{K}^{K} \mathcal{Y D}$ and $\mathcal{G}:{ }_{K}^{K} \mathcal{Y} \mathcal{D} \rightarrow{ }_{L}^{L} \mathcal{Y D}$ that preserve dimensions, gradings, Hopf algebras, spaces of primitive elements and Nichols algebras.

The implication $(\mathrm{b}) \Longrightarrow(\mathrm{a})$ is a consequence of Proposition 2.10 .

$(\mathrm{a}) \Longrightarrow(\mathrm{b})$ we consider the graded Hopf algebra $\mathcal{F}(R)=\oplus_{i \in \mathbb{N}_{0}} \mathcal{F}(R)^{i} \in$ ${ }_{K}^{K} \mathcal{Y} \mathcal{D}$, where $\mathcal{F}(R)^{i}=\mathcal{F}\left(R^{i}\right)$. Since $\mathcal{P}(\mathcal{F}(R))=\mathcal{F}(\mathcal{P}(R))$, Lemma 3.13 says that $\mathcal{P}(\mathcal{F}(R))=\mathcal{F}(R)^{1}=: Z$. Let $\mathrm{R}$ be the subalgebra of $\mathcal{F}(R)$ generated by $Z$. By Theorem 3.9, $Z$ is semisimple. Hence $Z=L\left(\lambda_{1}\right) \oplus \cdots \oplus L\left(\lambda_{t}\right)$ for some $\lambda_{1}, \ldots, \lambda_{t} \in \operatorname{Irr}_{\mathbb{k} G}^{\mathbb{k} G} \mathcal{Y} \mathcal{D}$ and (1.6) holds by Theorem 1.1 .

By Proposition 3.12, $\mathrm{R} \simeq \mathscr{B}(Z)$. By Remark 3.1, $\mathcal{F}(R) \# K$ is pointed; being finite-dimensional, it is generated by $G$ and the subspaces $\mathcal{P}_{g, 1}, g \in G$. By Lemma 3.10, $\mathcal{P}_{g, 1} \subset \mathcal{P}(\mathcal{F}(R)) \# 1+1 \# V$ for all $g \in G$. As $\mathcal{P}(\mathcal{F}(R))=Z$, $\mathcal{F}(R) \# K$ is generated by $Z, V$ and $G$; as $\mathbb{k}\langle Z, V, G\rangle=\mathrm{R} \# K$, we have that $\mathcal{F}(R) \# K=\mathrm{R} \# K$. That is, $\mathcal{F}(R) \simeq \mathscr{B}(Z)$. Therefore

$$
R \simeq \mathcal{G}(\mathcal{F}(R)) \simeq \mathcal{G}(\mathscr{B}(Z)) \simeq \mathscr{B}(\mathcal{Z}), \quad \mathcal{Z}=\mathcal{G}\left(L\left(\lambda_{1}\right)\right) \oplus \cdots \oplus \mathcal{G}\left(L\left(\lambda_{t}\right)\right),
$$

which completes the proof.

\section{Decompositions From diagonal type}

In this Section we compute some of the Nichols algebras $\mathscr{B}\left(Z_{U}\right)$ assuming that $W=V \oplus U$ is of diagonal type.

\subsection{Generalities.}


4.1.1. The setting. Let $\theta \in \mathbb{N}$ and $\mathbb{I}=\mathbb{I}_{\theta}$. Let $W$ be a braided vector space of diagonal type, of dimension $\theta$ with braiding matrix $\left(q_{i j}\right)_{i, j \in \mathbb{I}}$ with respect to a basis $\left(x_{i}\right)_{i \in \mathbb{I}}$. This matrix defines a $\mathbb{Z}$-bilinear form $\mathfrak{q}: \mathbb{Z}^{\mathbb{I}} \times \mathbb{Z}^{\mathbb{I}} \rightarrow \mathbb{K}^{\times}$by $\mathfrak{q}\left(\alpha_{j}, \alpha_{k}\right)=q_{j k}$ for all $j, k \in \mathbb{I}$. We set

$$
\mathfrak{q}_{\alpha \beta}=\mathfrak{q}(\alpha, \beta), \quad N_{\beta}=\operatorname{ord} \mathfrak{q}_{\beta \beta} \in \mathbb{N} \cup\{\infty\}, \quad \alpha, \beta \in \mathbb{Z}^{\mathbb{I}}, i \in \mathbb{I} .
$$

We assume that $q_{i i} \neq 1, i \in \mathbb{I}$ and set $\widetilde{q}_{i j}=q_{i j} q_{j i}, i<j$. We keep the notations from [AA2]. We describe the braiding of $W$ by its Dynkin diagram [H2], see e.g. [AA2, §2.1]; for instance, if $\theta=2, \begin{array}{rr}q_{11} \\ 1\end{array} \stackrel{\widetilde{q}_{12}}{\underset{1}{q_{22}}} \begin{array}{r}q_{2} \\ 2\end{array}$.

Let $\Gamma$ be a free abelian group of rank $\theta$ with a fixed basis $\left(g_{i}\right)_{i \in \mathbb{I}_{\theta}}$. We define $\chi_{i}: \Gamma \rightarrow \mathbb{k}^{\times}$by $\chi_{i}\left(g_{j}\right)=q_{j i}$. We realize $W \in{ }_{\mathbb{k} \Gamma}^{\mathbb{k} \Gamma} \mathcal{Y} \mathcal{D}$ by declaring that

$$
x_{i} \in W_{g_{i}}^{\chi_{i}}, \quad i \in \mathbb{I}_{\theta} \text {. }
$$

As in AA2, we set

$$
\begin{aligned}
x_{i j} & =\operatorname{ad}_{c} x_{i}\left(x_{j}\right)=x_{i} x_{j}-q_{i j} x_{j} x_{i}, & & i \neq j \in \mathbb{I}, \\
x_{i_{1} i_{2} \cdots i_{k}} & =\left(\operatorname{ad}_{c} x_{i_{1}}\right) \cdots\left(\operatorname{ad}_{c} x_{i_{k-1}}\right)\left(x_{i_{k}}\right), & & i_{1}, i_{2}, \cdots, i_{k} \in \mathbb{I}, \\
x_{(k l)} & =x_{k(k+1)(k+2) \ldots l}, & & k<l \in \mathbb{I} .
\end{aligned}
$$

We fix $t \in \mathbb{I}_{\theta-1}$; let $V$, respectively $U$, be the subspace generated by $\left(x_{i}\right)_{i \in \mathbb{I}_{t}}$, respectively $\left(x_{i}\right)_{i \in \mathbb{I}_{t+1, \theta}}$. We keep the notation in (2.13). Then

$$
\mathscr{B}(W) \simeq \mathscr{B}\left(Z_{U}\right) \# \mathscr{B}(V) \simeq \mathscr{B}\left(Z_{V}\right) \# \mathscr{B}(U) .
$$

4.1.2. Assumptions. We assume that $W$ is arithmetic, i.e. that the set $\Delta_{+}$ of positive roots of $W$ is finite, see [AA2, §2.4]. Then the $n_{i j}$ 's defined below are integers, i.e. the sets on the right are non-empty:

$$
n_{i j}:=\min \left\{n \in \mathbb{N}_{0}:(n+1)_{q_{i i}}\left(1-q_{i i}^{n} q_{i j} q_{j i}\right)=0\right\}, \quad i \neq j \in \mathbb{I} .
$$

We fix a convex order of $\Delta_{+}$An1, see AA2, Theorem 2.36]. Correspondingly, we have the numeration $\left(\beta_{k}\right)_{k \in \mathbb{I}_{\ell}}$ of $\Delta_{+}$. For every $k \in \mathbb{I}_{\ell}$, let $x_{\beta_{k}}$ be the corresponding root vector; these are defined as in AA2, Remark 2.14] when the convex order arises from Lyndon words, or via the Lusztig isomorphisms in general. These generate a PBW-basis of $\mathscr{B}(W)$, that is

$$
\left\{x_{\beta_{\ell}}^{n_{\ell}} x_{\beta_{\ell-1}}^{n_{\ell-1}} \ldots x_{\beta_{2}}^{n_{2}} x_{\beta_{1}}^{n_{1}} \mid n_{k} \in \mathbb{I}_{0, N_{\beta_{k}}-1}, k \in \mathbb{I}_{\ell}\right\} .
$$

Let $i<j \in \mathbb{I}_{\ell}, p_{i+1}, \ldots, p_{j-1} \in \mathbb{N}_{0}$. Since the order is convex, there exist $c_{p_{i+1}, \ldots, p_{j-1}}^{(i, j)} \in \mathbb{k}$, explicitly computable [An1, Lemma 4.5], such that

$$
\left[x_{\beta_{i}}, x_{\beta_{j}}\right]_{c}=\sum_{p_{i+1}, \ldots, p_{j-1} \in \mathbb{N}_{0}} c_{p_{i+1}, \ldots, p_{j-1}}^{(i, j)} x_{\beta_{j-1}}^{p_{j-1}} \ldots x_{\beta_{i+1}}^{p_{i+1}} .
$$

Notice that if $\sum p_{k} \beta_{k} \neq \beta_{i}+\beta_{j}$, then $c_{p_{i+1}, \ldots, p_{j-1}}^{(i, j)}=0$, since $\mathscr{B}_{\mathfrak{q}}$ is $\mathbb{N}_{0}^{\mathbb{I}}$-graded.

Remark 4.1. Let $j=i+1 \in \mathbb{I}_{\ell}$. Then $\left[x_{\beta_{i}}, x_{\beta_{j}}\right]_{c}=0$ by (4.5). 
4.1.3. Properties of $\mathscr{B}\left(Z_{U}\right)$. We first describe a basis of $\mathscr{B}\left(Z_{U}\right)$. Given $\alpha=$ $\sum_{i \in \mathbb{I}} c_{i} \alpha_{i} \in \mathbb{Z}^{\mathbb{I}}$, let $\operatorname{supp} \alpha=\left\{i \in \mathbb{I}: c_{i} \neq 0\right\}$. Let $\Delta_{+}^{V}$ be the set of positive roots of $V, \mathfrak{l}=\left|\Delta_{+}^{V}\right|$. Hence $\Delta_{+}^{V}=\left\{\alpha \in \Delta_{+}: \operatorname{supp} \alpha \subset \mathbb{I}_{t}\right\}$.

Lemma 4.2. There exists a convex order $<$ on $\Delta_{+}$such that $\Delta_{+}^{V}=\left\{\beta_{k}\right\}_{k \in \mathbb{I}_{\mathfrak{l}}}$.

Proof. Here we shall use the notation of [AA2, §2.7]; in particular, the Weyl groupoid of $V$ is denoted $\mathcal{W}_{V}$. Let $\omega_{0}^{V}$ be the element of maximal length of $\mathcal{W}_{V}$ that ends at $V$. We fix a reduced expression $\omega_{0}^{V}=\sigma_{i_{1}}^{V} \sigma_{i_{2}} \ldots \sigma_{i_{\mathrm{I}}}, i_{j} \in \mathbb{I}_{t}$. By abuse of notation, we consider the element $\omega=\sigma_{i_{1}}^{W} \sigma_{i_{2}} \ldots \sigma_{i_{1}}$ of $\mathcal{W}_{W}$ that ends at $W$. Clearly, $\omega$ has length $\mathfrak{l}$, that is, the expression $\sigma_{i_{1}}^{W} \sigma_{i_{2}} \ldots \sigma_{i_{\mathfrak{l}}}$ since $s_{i_{1}}^{W} \ldots s_{i_{j-1}}\left(\alpha_{i_{j}}\right)=s_{i_{1}}^{V} \ldots s_{i_{j-1}}\left(\alpha_{i_{j}}\right)$ is a positive root for all $j \in \mathbb{I}_{\mathfrak{l}}$, cf. [HY2, Corollary 3]. Using [HY2, Corollary 4] we may extend this expression to an expression of the element of maximal length $\omega_{0}^{W}=\sigma_{i_{1}}^{W} \ldots \sigma_{i_{\mathfrak{l}}} \sigma_{i_{\mathrm{l}+1}} \ldots \sigma_{i_{\ell}}$; hence the first $\mathfrak{l}$ roots of the associated convex order are the roots in $\Delta_{+}^{V}$.

By a combination of the main results of [U1, [U2], the algebra $\mathscr{B}\left(Z_{U}\right)$ has a PBW-basis. We next give an explicit choice of such a basis.

Proposition 4.3. The elements $x_{\beta_{k}}$ with $k \in \mathbb{I}_{\mathfrak{l}+1, \ell}$ generate a PBW-basis of $\mathscr{B}\left(Z_{U}\right)$. That is, the following set is a basis of $\mathscr{B}\left(Z_{U}\right)$ :

$$
\left\{x_{\beta_{\ell}}^{n_{\ell}} x_{\beta_{\ell-1}}^{n_{\ell-1}} \ldots x_{\beta_{\mathfrak{l}+1}}^{n_{\mathfrak{l}+1}} \mid n_{k} \in \mathbb{I}_{0, N_{\beta_{k}}-1}, k \in \mathbb{I}_{\mathfrak{l}+1, \ell}\right\} .
$$

The order of $\mathbb{I}_{\mathfrak{l}+1, \ell}$ defines a filtration on $\mathscr{B}\left(Z_{U}\right)$ whose associated graded algebra is a (truncated) quantum polynomial ring.

Proof. Let $i \in \mathbb{I}_{t}, j \in \mathbb{I}_{\mathfrak{l}+1, \ell}$. Suppose that $\partial_{i}\left(x_{\beta_{j}}\right) \neq 0$ : that is, some $x_{\beta_{\ell}}^{m_{\ell}} x_{\beta_{\ell-1}}^{m_{\ell-1}} \ldots x_{\beta_{1}}^{m_{1}} \otimes x_{i}$ appears with non-zero coefficient in the expression of $\Delta\left(x_{\beta_{j}}\right)$. The subspace spanned by $\left\{x_{\beta_{j}}^{n_{j}} x_{\beta_{j-1}}^{n_{j-1}} \ldots x_{\beta_{1}}^{n_{1}} \mid n_{k} \in \mathbb{I}_{0, N_{\beta_{k}}}, k \in \mathbb{I}_{j}\right\}$ is a right coideal subalgebra [HS1], so $m_{k}=0$ for all $k \in \mathbb{I}_{j+1, \ell}$; as the coproduct preserves the $\mathbb{Z}^{\mathbb{I}}$-degree, we also have $m_{j}=0$, and

$$
\beta_{j}=\alpha_{i}+\sum_{k \in \mathbb{I}_{j-1}} m_{k} \beta_{k}
$$

Note that $\alpha_{i}=\beta_{k}$ for some $k \in \mathbb{I}_{j-1}$, see Lemma 4.2. As the order is strongly convex An1, Theorem 3.11], we have that $\beta_{1}<\beta_{j}<\beta_{j-1}$, a contradiction. Hence $\partial_{i}\left(x_{\beta_{j}}\right)=0$ for all $i \in \mathbb{I}_{t}$, and by Proposition 2.7, $x_{\beta_{j}} \in \mathscr{B}\left(Z_{U}\right)$.

As $\mathscr{B}\left(Z_{U}\right)$ is a subalgebra of $\mathscr{B}(W)$, every element in (4.6) belongs to $\mathscr{B}\left(Z_{U}\right)$. The set (4.6) is linearly independent and spans a subspace with the same Hilbert series as $\mathscr{B}\left(Z_{U}\right)$, hence it is a basis of $\mathscr{B}\left(Z_{U}\right)$.

The last statement follows e.g. as [An3, Proposition 16, Corollary 17]. 
A first immediate consequence is that $\mathscr{B}\left(Z_{U}\right)$ is noetherian. If $W$ is generic, hence of Cartan type [AA1, R], then it satisfies further properties.

Theorem 4.4. Assume that $W$ is generic. The algebra $\mathscr{B}\left(Z_{U}\right)$ is an iterated Ore extension; thus it is strongly noetherian, AS-regular and CohenMacaulay.

In many cases the Theorem was already known, e.g. ArS, (0.2), (8.5)], [NS, Remark 2.9], [E, Theorem 4.3], [LW, §7].

Proof. First, we check that $\mathscr{B}\left(Z_{U}\right)$ is an iterated Ore extension. In fact, for each $j \in\{\mathfrak{l}+1, \ell\}$ let $R_{j}$ be the subspace spanned by

$$
\left\{x_{\beta_{\ell}}^{n_{\ell}} x_{\beta_{\ell-1}}^{n_{\ell-1}} \ldots x_{\beta_{j}}^{n_{j}} \mid n_{k} \in \mathbb{I}_{0, N_{\beta_{k}}}, k \in \mathbb{I}_{\mathfrak{l}+1, \ell}\right\} .
$$

Thus each $R_{j}$ is a free $R_{j+1}$-module with basis $\left(x_{\beta_{j}}^{n}\right)_{n \in \mathbb{N}_{0}}$. Let $\sigma_{j}$ be the algebra automorphism given by the action of $g_{j}$, and $D_{j}:=\left[x_{\beta_{j}},-\right]_{c}$. Then $D_{j}$ is a (id, $\left.\sigma_{j}\right)$-derivation, and by (4.5), $D_{j}\left(x_{\beta_{k}}\right) \in R_{j+1}$ for all $k \in \mathbb{I}_{j+1, \ell}$. Hence

$$
\left[x_{\beta_{j}}, x\right]_{c}=x_{\beta_{j}} x-\left(g_{j} \cdot x\right) x_{\beta_{j}} \in R_{j+1} \quad \text { for all } x \in R_{j+1},
$$

and $D_{j}$ restricts to a (id, $\left.\sigma_{j}\right)$-derivation of $R_{j+1}$. Therefore

$$
R_{j} \simeq R_{j+1}\left[x_{\beta_{j}} ; \sigma_{j}, D_{j}\right], \quad \text { for all } j \in \mathbb{I}_{\mathfrak{l}+1, \ell-1} .
$$

Now $\mathscr{B}\left(Z_{U}\right)$ is strongly noetherian by [ASZ, Proposition 4.10]; AS-regular by [AST, Proposition 2] and Cohen-Macaulay by [ZZ, Lemma 5.3].

Next we deal with the Laistrygonian Nichols algebras $\mathscr{B}(\mathfrak{L}(1, \mathscr{G}))$ AAH1.

Theorem 4.5. The algebra $\mathscr{B}(\mathfrak{L}(1, \mathscr{G}))$ is an iterated Ore extension; thus it is strongly noetherian, AS-regular and Cohen-Macaulay.

Proof. We follow the notation of [AAH1, §4.3]. The subalgebra of $\mathscr{B}(\mathfrak{L}(1, \mathscr{G}))$ generated by $x_{1}, x_{2}$ is (isomorphic to) the Jordan plane, so it is an Ore extension of the polynomial algebra in one variable.

For each $j \in \mathbb{I}_{0, \mathscr{G}}$, let $R_{j}$ be the subalgebra generated by $x_{1}, x_{2}$ and $z_{k}$, $k \in \mathbb{I}_{j, \mathscr{G}}$. By [AAH1, Proposition 4.16], each $R_{j}$ is a free $R_{j+1}$-module with basis $\left(z_{j}^{n}\right)_{n \in \mathbb{N}_{0}}$. Let $\sigma_{j}$ the algebra automorphism given by the action of $g_{1}^{j} g_{2}$, and $D_{j}:=\left[z_{j},-\right]$. Then $D_{j}$ is a $\left(\mathrm{id}, \sigma_{j}\right)$-derivation, and by AAH1, Lemmas 4.14, 4.15], $D_{j}$ restricts to a (id, $\sigma_{j}$ )-derivation of $R_{j+1}$. Thus $R_{j} \simeq$ $R_{j+1}\left[z_{j} ; \sigma_{j}, D_{j}\right]$, for all $j \in \mathbb{I}_{0, \mathscr{G}}$. Now $\mathscr{B}(\mathfrak{L}(1, \mathscr{G}))$ is strongly noetherian by [ASZ, Proposition 4.10]; AS-regular by [AST, Proposition 2] and CohenMacaulay by [ZZ, Lemma 5.3]. 
4.1.4. Organization of the Section. In the rest of the Section we present the defining relations of some specific $\mathscr{B}\left(Z_{U}\right)$. We state now some terminology for general use. First, in 4.2 we deal with the case when $\operatorname{dim} W=2$ and the braiding is of Cartan type. The same method works routinely for other arithmetic root systems of rank 2 .

Assume now that $\theta>2$. Given $\mathbb{J} \subset \mathbb{I}_{\theta}$, we set

$$
\begin{aligned}
& V_{\mathbb{J}}=\oplus_{j \in \mathbb{J}} \mathbb{k} x_{j}, \quad U_{\mathbb{J}}=\oplus_{h \notin \mathbb{J}} \mathbb{k} x_{h}, \quad \text { so that } \quad W=V_{\mathbb{J}} \oplus U_{\mathbb{J}}, \\
& Z_{U_{\mathbb{J}}}=\operatorname{ad}_{c} \mathscr{B}\left(V_{\mathbb{J}}\right)\left(U_{\mathbb{J}}\right)=\oplus_{h \notin \mathbb{J}} \text { ad } \mathscr{B}\left(V_{\mathbb{J}}\right) x_{h} .
\end{aligned}
$$

Observe that $Z_{V_{\mathrm{J}}}=Z_{U_{\mathbb{J}^{\prime}}}$, where $\mathbb{J}^{\prime}=\mathbb{I}_{\theta}-\mathbb{J}$ (thus we do not need to consider $Z_{V_{\mathbb{J}}}$ ) and that $Z_{U_{\mathrm{J}}} \simeq Z_{U_{\vartheta(\mathbb{J})}}$ as braided vector spaces whenever $\vartheta$ is an automorphism of the Dynkin diagram [AA2, §2.1]. We are mostly interested in the cases when $\mathbb{I}$ consists of an extremal vertex or its complement. Indeed $Z_{U_{\mathbb{J}}}$ would turn out to be simple exactly when card $\mathbb{J}=1$, and $\mathscr{B}\left(V_{\mathbb{J}}\right)$ would give rise to $U_{q}(\mathfrak{g})$ with $\mathfrak{g}$ simple exactly when the Dynkin diagram of $V_{\mathbb{J}}$ is connected.

4.2. Dimension $W=2$. Here $V=\mathbb{k} x_{1}$ and $U=\mathbb{k} x_{2}$ have dimension 1 . Then $\operatorname{dim} Z_{U}=n_{12}+1$ and $\operatorname{dim} Z_{V}=n_{21}+1 ;$ more precisely,

$$
\begin{aligned}
Z_{U} & =\operatorname{ad}_{c} \mathscr{B}(V)(U)=\oplus_{n \in \mathbb{I}_{0, n_{12}}} \mathbb{k} u_{n}, \quad u_{n}:=\operatorname{ad}_{c}\left(x_{1}\right)^{n}\left(x_{2}\right) ; \\
u_{n+1} & =x_{1} u_{n}-q_{11}^{n} q_{12} u_{n} x_{1} .
\end{aligned}
$$

Notice that $u_{n}=x_{n \alpha_{1}+\alpha_{2}}$ belongs to the PBW-basis alluded above. Let

$$
A_{n}=\prod_{j \in \mathbb{I}_{0, n-1}}\left(1-q_{11}^{j} \widetilde{q}_{12}\right), \quad \text { so that } \quad n \leq n_{12} \Longrightarrow A_{n} \neq 0 .
$$

Lemma 4.6. The braiding of $Z_{U}$ is given by

$$
\begin{aligned}
& c\left(u_{n} \otimes u_{m}\right)=\sum_{j \in \mathbb{I}_{0, n}: m+j \leq n_{12}} q_{11}^{m(n-j)} q_{12}^{n-j} q_{21}^{m} q_{22}\left(\begin{array}{c}
n \\
j
\end{array}\right)_{q_{11}} \frac{A_{n}}{A_{n-j}} u_{m+j} \otimes u_{n-j}, \\
& n, m \in \mathbb{I}_{0, n_{12}} .
\end{aligned}
$$

In particular, $Z_{U}$ is of diagonal type if and only if $n_{12}=0$, hence also $n_{21}=0$.

Proof. Recall the realization in 4.1 The coaction $\delta: Z_{U} \rightarrow \mathscr{B}(V) \# \mathbb{k} \Gamma \otimes Z_{U}$ is given by (2.12). Hence $\delta\left(u_{0}\right)=\alpha_{2} \otimes u_{0}$. We claim that for every $n \in \mathbb{I}_{0, n_{12}}$,

$$
\delta\left(u_{n}\right)=\sum_{i \in \mathbb{I}_{0, n}}\left(\begin{array}{l}
n \\
i
\end{array}\right)_{q_{11}} \frac{A_{n}}{A_{i}} x_{1}^{n-i} g_{1}^{i} g_{2} \otimes u_{i} .
$$

The proof of (4.9) goes by induction on $n$. Indeed, by (4.7), we have

$$
\delta\left(u_{n+1}\right)=\left(x_{1} \otimes 1+g_{1} \otimes x_{1}\right) \delta\left(u_{n}\right)-q_{11}^{n} q_{12} \delta\left(u_{n}\right)\left(x_{1} \otimes 1+g_{1} \otimes x_{1}\right) .
$$


Then (4.8) follows. We next claim that

$$
\exists q \in \mathbb{k}^{\times}, x \in Z_{U}: c(x \otimes x)=q x \otimes x \Longleftrightarrow x \in \mathbb{k} u_{0} \text { or } x \in \mathbb{k} u_{n_{12}} .
$$

Assume that there exist such $q$ and $x$ and write

$$
x=\sum_{i \in \mathbb{I}_{n, p}} c_{i} u_{i}, \quad n \leq p, c_{n}, c_{p} \neq 0 .
$$

Let $Q_{t}^{i, j}=q_{11}^{j(i-t)} q_{12}^{i-t} q_{21}^{j} q_{22}\left(\begin{array}{l}i \\ t\end{array}\right)_{q_{11}} \frac{A_{i}}{A_{i-t}}$. Then

$$
\begin{aligned}
\sum_{\substack{i, j \in \mathbb{I}_{n, p}, t \in \mathbb{I}_{0, i}: j+t \leq n_{12}}} c_{i} c_{j} Q_{t}^{i, j} u_{j+t} \otimes u_{i-t} & =\sum_{r, s \in \mathbb{I}_{n, p}} q c_{r} c_{s} u_{r} \otimes u_{s} ; \\
\Longrightarrow \sum_{\substack{i, j \in \mathbb{I}_{n, p}, 0 \leq t \leq \min \left\{i, n_{12}-j\right\} \\
j+t=r, i-t=s}} c_{i} c_{j} Q_{t}^{i, j} u_{j+t} \otimes u_{i-t} & =q c_{r} c_{s} u_{r} \otimes u_{s} \quad \forall r, s \in \mathbb{I}_{n, p} .
\end{aligned}
$$

Take $(r, s)=(n, p)$. Then $j=n, i=p$ and necessarily $t=0$; the last could only happen if $\min \left\{p, n_{12}-n\right\}=0$. That is, either $p=0=n$, or else $n=n_{12}=p$. This shows (4.10).

Thus, if $Z_{U}$ is diagonal type, then $n_{12} \leq 1$. If $n_{12}=1$, then

$$
\begin{aligned}
& c\left(u_{0} \otimes u_{1}\right)=q_{21} q_{22} u_{1} \otimes u_{0}, \\
& c\left(u_{1} \otimes u_{0}\right)=q_{12} q_{22} u_{0} \otimes u_{1}+q_{22}\left(1-\widetilde{q}_{12}\right) u_{1} \otimes u_{0}
\end{aligned}
$$

by a similar computation. Here $\widetilde{q}_{12} \neq 1$ since $n_{12} \neq 0$; hence $Z_{U}$ is not of diagonal type. We conclude that $n_{12}=0$.

4.2.1. Cartan type $A_{2}$. Here $q \in \mathbb{k}^{\times}-\{1\}$. The Dynkin diagram is $\underset{1}{\stackrel{q}{\circ}} \stackrel{q^{-1}}{\stackrel{q}{\circ}} \underset{2}{q}$ and

$$
\Delta_{+}=\left\{\beta_{1}=\alpha_{1}, \beta_{2}=\alpha_{1}+\alpha_{2}, \beta_{3}=\alpha_{2}\right\} .
$$

Thus $Z_{U}=\mathbb{k} u_{0} \oplus \mathbb{k} u_{1} ; u_{0}=x_{\beta_{3}}, u_{1}=x_{\beta_{2}}$. By Remark 4.1. $\left[u_{1}, u_{0}\right]_{c}=0$. Hence

$$
\mathscr{B}\left(Z_{U}\right)=\mathbb{k}\left\langle u_{0}, u_{1} \mid u_{1} u_{0}-q_{12} q u_{0} u_{1}, u_{0}^{N}, u_{1}^{N}\right\rangle,
$$

where $N=\operatorname{ord} q$. We omit the last relations when $N=\infty$, in which case:

Proposition 4.7. If $W$ is of type $A_{2}$ and $q \notin \mathbb{G}_{\infty}$, then $\mathscr{B}\left(Z_{U}\right)$ is a quantum plane, with $\mathrm{GK}-\operatorname{dim}=2$.

It is well-known that in this case, $\mathscr{B}\left(Z_{U}\right)$ is AS-regular, see [ArS, (0.2)]. 


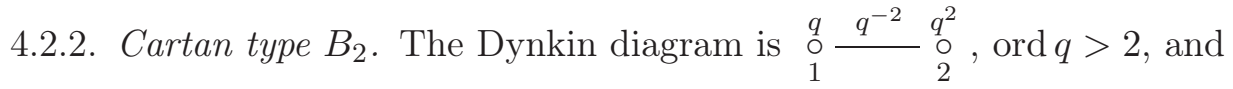

$$
\Delta_{+}=\left\{\beta_{1}=\alpha_{1}, \beta_{2}=2 \alpha_{1}+\alpha_{2}, \beta_{3}=\alpha_{1}+\alpha_{2}, \beta_{4}=\alpha_{2}\right\} .
$$

Hence $Z_{U}=\mathbb{k} u_{0} \oplus \mathbb{k} u_{1} \oplus \mathbb{k} u_{2} ; u_{i}=x_{\beta_{4-i}}, i \in \mathbb{I}_{0,2}$. By Remark 4.1,

$$
u_{1} u_{0}=q^{2} q_{12} u_{0} u_{1}, \quad u_{2} u_{1}=q^{2} q_{12} u_{1} u_{2} .
$$

We check that

$$
u_{2} u_{0}=q^{2} q_{12}^{2} u_{0} u_{2}+q q_{12}(q-1) u_{1}^{2} .
$$

Thus $\mathscr{B}\left(Z_{U}\right)$ is presented by generators $u_{i}, i \in \mathbb{I}_{0,2}$, and relations (4.11), (4.12),

$$
u_{0}^{M}=0, \quad u_{1}^{N}=0, \quad u_{2}^{M}=0,
$$

where $N=\operatorname{ord} q, M=\operatorname{ord} q^{2}$. Particularly, when $N=\infty$, we have:

Proposition 4.8. If $W$ is of type $B_{2}$ and $q \notin \mathbb{G}_{\infty}$, then $\mathscr{B}\left(Z_{U}\right)$ is a quadratic algebra with relations (4.11), (4.12), and $\mathrm{GK}$-dim $=3$.

In this case, it is known that $\mathscr{B}\left(Z_{U}\right)$ is AS-regular, see [NS, Remark 2.9].

4.2.3. Cartan type $C_{2}$. The Dynkin diagram is $\underset{1}{\stackrel{q^{2}}{0}} \stackrel{q^{-2}}{\underset{1}{0}} \underset{2}{q}$, ord $q>2$, and

$$
\Delta_{+}=\left\{\beta_{1}=\alpha_{1}, \beta_{2}=\alpha_{1}+\alpha_{2}, \beta_{3}=\alpha_{1}+2 \alpha_{2}, \beta_{4}=\alpha_{2}\right\} .
$$

Hence $Z_{U}=\mathbb{k} u_{0} \oplus \mathbb{k} u_{1}, u_{0}=x_{\beta_{4}}, u_{1}=x_{\beta_{2}}$. Set $u_{10}=u_{1} u_{0}-q_{12} q u_{0} u_{1}=x_{\beta_{3}}$. By Remark 4.1.

$$
u_{1} u_{10}=q^{2} q_{12} u_{10} u_{1}, \quad u_{10} u_{0}=q^{2} q_{12} u_{0} u_{10} .
$$

Thus $\mathscr{B}\left(Z_{U}\right)$ is presented by generators $u_{0}, u_{1}$, and relations (4.13),

$$
u_{0}^{N}=0, \quad u_{10}^{M}=0, \quad u_{1}^{N}=0,
$$

where $N=\operatorname{ord} q, M=\operatorname{ord} q^{2}$. Clearly (4.13) are quantum Serre relations.

Remark 4.9. If $N<\infty$, then $\mathscr{B}\left(Z_{U}\right)$ is isomorphic as an algebra to a Nichols algebra of diagonal type only when $M=N$.

Particularly, when $N=\infty$, we have:

Proposition 4.10. If $W$ is of type $C_{2}$ and $q \notin \mathbb{G}_{\infty}$, then $\mathscr{B}\left(Z_{U}\right)$ is a cubic algebra with relations (4.13), and GK-dim $=3$.

In this case, it is known that $\mathscr{B}\left(Z_{U}\right)$ is AS-regular, see [ArS, (8.5)]. 
4.2.4. Cartan type $G_{2}$. The Dynkin diagram is $\underset{1}{\stackrel{q}{O}} \stackrel{q^{-3}}{\underset{0}{\circ}} \underset{2}{q^{3}}$, ord $q>3$, and

$$
\begin{gathered}
\Delta_{+}=\left\{\beta_{1}=\alpha_{1}, \beta_{2}=3 \alpha_{1}+\alpha_{2}, \beta_{3}=2 \alpha_{1}+\alpha_{2},\right. \\
\left.\beta_{4}=3 \alpha_{1}+2 \alpha_{2}, \beta_{5}=\alpha_{1}+\alpha_{2}, \beta_{6}=\alpha_{2}\right\} .
\end{gathered}
$$

Hence $Z_{U}=\mathbb{k} u_{0} \oplus \mathbb{k} u_{1} \oplus \mathbb{k} u_{2} \oplus \mathbb{k} u_{3}, u_{0}=x_{\beta_{6}}, u_{1}=x_{\beta_{5}}, u_{2}=x_{\beta_{3}}, u_{3}=x_{\beta_{2}}$. Set $u_{21}=u_{2} u_{1}-q^{2} q_{12} u_{1} u_{2}=x_{\beta_{4}}$. By Remark 4.1.

$$
u_{1} u_{0}=q^{3} q_{12} u_{0} u_{1}, \quad u_{3} u_{2}=q^{3} q_{12} u_{2} u_{3} .
$$

We check that

$$
\begin{aligned}
& u_{3} u_{1}=q^{3} q_{12}^{2} u_{1} u_{3}+\frac{q q_{12}\left(q^{3}-1\right)}{q+1} u_{2}^{2}, \\
& u_{3} u_{0}=q^{3} q_{12}^{3} u_{0} u_{3}+q_{12}\left(q^{3}-q^{2}-q\right) u_{21}+q q_{12}(1-q)(3)_{q}^{!} u_{1} u_{2}, \\
& u_{2} u_{0}=q^{3} q_{12}^{2} u_{0} u_{2}+q q_{12}\left(q^{2}-1\right) u_{1}^{2}, \\
& u_{2} u_{21}=q^{3} q_{12} u_{21} u_{2}, \\
& u_{21} u_{1}=q^{3} q_{12} u_{1} u_{21} .
\end{aligned}
$$

Thus $\mathscr{B}\left(Z_{U}\right)$ is generated by $u_{i}, i \in \mathbb{I}_{0,3}$, with relations (4.14), (4.15), (4.16),

$$
u_{0}^{M}=0, \quad u_{1}^{N}=0, \quad u_{21}^{M}=0, \quad u_{2}^{N}=0, \quad u_{3}^{M}=0,
$$

where $N=\operatorname{ord} q, M=\operatorname{ord} q^{3}$. Particularly, when $N=\infty$, we have:

Proposition 4.11. If $W$ is of type $G_{2}$ and $q \notin \mathbb{G}_{\infty}$, then $\mathscr{B}\left(Z_{U}\right)$ has quadratic relations (4.14), (4.15) and cubic relations (4.16); also, GK-dim $=5$.

4.2.5. Cartan type $G_{2}$ with the inverse numeration. The Dynkin diagram is

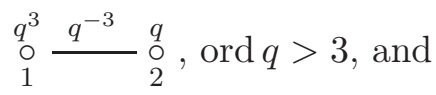

$$
\begin{array}{r}
\Delta_{+}=\left\{\beta_{1}=\alpha_{1}, \beta_{2}=\alpha_{1}+\alpha_{2}, \beta_{3}=2 \alpha_{1}+3 \alpha_{2},\right. \\
\left.\beta_{4}=\alpha_{1}+2 \alpha_{2}, \beta_{5}=\alpha_{1}+3 \alpha_{2}, \beta_{6}=\alpha_{2}\right\} .
\end{array}
$$

Hence $Z_{U}=\mathbb{k} u_{0} \oplus \mathbb{k} u_{1}, u_{0}=x_{\beta_{6}}, u_{1}=x_{\beta_{2}}$. Set $u_{10}=u_{1} u_{0}-q q_{12} u_{0} u_{1}=x_{\beta_{4}}$, $u_{100}=u_{10} u_{0}-q^{2} q_{12} u_{0} u_{10}=x_{\beta_{5}}, u_{110}=u_{1} u_{10}-q^{2} q_{12} u_{10} u_{1}=x_{\beta_{3}}$. Ву Remark 4.1.

$$
u_{1} u_{110}=q^{3} q_{12} u_{110} u_{1}, \quad u_{100} u_{0}=q^{3} q_{12} u_{0} u_{100} .
$$

We check that

$$
u_{1} u_{100}=q^{3} q_{12}^{2} u_{100} u_{1}+\frac{q_{12}(3)_{q}(q-1)^{2}}{q+1} u_{10}^{2} .
$$

Thus $\mathscr{B}\left(Z_{U}\right)$ is presented by generators $u_{0}, u_{1}$, and relations (4.17), (4.18),

$$
u_{0}^{N}=0, \quad u_{110}^{M}=0, \quad u_{10}^{N}=0, \quad u_{100}^{M}=0, \quad u_{1}^{N}=0,
$$

where $N=\operatorname{ord} q, M=\operatorname{ord} q^{3}$. When $N=\infty$, we have: 
Proposition 4.12. If $W$ is of type $G_{2}$ and $q \notin \mathbb{G}_{\infty}$, then $\mathscr{B}\left(Z_{U}\right)$ is defined by the quartic relations (4.17), (4.18); also, GK-dim $=5$.

In this case, $\mathscr{B}\left(Z_{U}\right)$ is AS-regular; it is the Algebra $F$ in [WW, §4].

4.3. Cartan type $A_{\theta}, \theta>2$. Here $q \in \mathbb{k}^{\times}-\{1\}$. The Dynkin diagram is

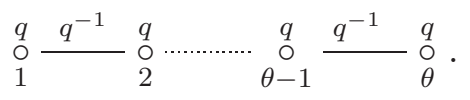

With the notation (1.11) and ordered lexicographically, the positive roots are

$$
\Delta_{+}=\left\{\alpha_{k j} \mid k \leq j \in \mathbb{I}\right\} .
$$

The generators of the PBW-basis are $x_{\alpha_{k k}}=x_{k}, x_{\alpha_{k l}}=x_{(k l)}, k<l$, cf. (4.3). Let $N=$ ord $q$. Assume that $q \neq-1$. The defining relations are

$$
\begin{aligned}
x_{i j} & =0, & i & <j-1, \\
x_{i i j} & =0, & |j-i| & =1, \\
x_{(k l)}^{N} & =0, & k & \leq l,
\end{aligned}
$$

where $i, j, k, l \in \mathbb{I}$. When $q=-1$, the defining relations are (4.20), (4.22) (with $N=2$ ), and

$$
\left[x_{(i-1, i+1)}, x_{i}\right]_{c}=0,
$$

$i \in \mathbb{I}_{2, \theta-1}$. As usual we omit (4.22) when $q \notin \mathbb{G}_{\infty}$.

4.3.1. $\mathbb{J}=\{1\}$. The algebra $\mathscr{B}\left(Z_{U}\right)$ is presented by $x_{12}, x_{2}, x_{3}, \ldots, x_{\theta}$ with defining relations (4.20), $i, j \in \mathbb{I}_{2, \theta}$; (4.21) if $q \neq-1$, or (4.23) if $q=-1$, $i, j \in \mathbb{I}_{2, \theta} ;$ (4.22) $, k, l \in \mathbb{I}_{2, \theta} ;$ and the new relations

$$
\begin{array}{rlrl}
{\left[x_{12}, x_{i}\right]_{c}} & =0, & i & \in\{2\} \cup \mathbb{I}_{4, \theta}, \\
{\left[x_{12},\left[x_{12}, x_{3}\right]_{c}\right]_{c}} & =0, & & \\
{\left[\left[x_{12}, x_{3}\right]_{c}, x_{2}\right]_{c}} & =0, & & \\
x_{12}^{N} & =0, & l \in \mathbb{I}_{3, \theta} .
\end{array}
$$

We omit the last relations when $N=\infty$, in which case $\mathscr{B}\left(Z_{U}\right)$ is a domain and $\mathrm{GK}-\operatorname{dim} \mathscr{B}\left(Z_{U}\right)=\left(\begin{array}{c}\theta+1 \\ 2\end{array}\right)-1$.

4.3.2. $\operatorname{card} \mathbb{J}=1$. The case $\mathbb{J}=\{\theta\}$ reduces to the previous one. To exemplify, we discuss only the case $\theta=3, \mathbb{J}=\{2\}$. The algebra $\mathscr{B}\left(Z_{U}\right)$ is presented by $x_{1}, x_{21}, x_{23}, x_{3}$ with defining relations

$$
\begin{aligned}
& {\left[x_{1}, x_{3}\right]_{c}=0, \quad\left[x_{1}, x_{21}\right]_{c}=0, \quad\left[x_{23}, x_{3}\right]_{c}=0, \quad\left[x_{21}, x_{23}\right]_{c}=0,} \\
& {\left[x_{21}, x_{3}\right]_{c}=q_{13}(1-q) x_{23} x_{1}-q_{21}\left[x_{1}, x_{23}\right]_{c},} \\
& x_{12}^{N}=0, \quad x_{21}^{N}=0, \quad x_{23}^{N}=0, \quad x_{3}^{N}=0, \quad\left[x_{1}, x_{23}\right]_{c}^{N}=0 .
\end{aligned}
$$


4.3.3. $\mathbb{I}=\mathbb{I}_{\theta-1}$. The algebra $\mathscr{B}\left(Z_{U}\right)$ is presented by $x_{(1 \theta)}, x_{(2 \theta)}, \ldots, x_{\theta}$ with defining relations

$$
\begin{aligned}
{\left[x_{(i \theta)}, x_{(j \theta)}\right]_{c} } & =0, & & i<j \in \mathbb{I}_{\theta}, \\
x_{(i \theta)}^{N} & =0, & & i \in \mathbb{I}_{\theta} .
\end{aligned}
$$

We omit the last relations when $N=\infty$, in which case $\mathscr{B}\left(Z_{U}\right)$ is a quantum linear space and GK-dim $\mathscr{B}\left(Z_{U}\right)=\theta$.

4.4. Cartan type $B_{\theta}, \theta>2$. Here $q \in \mathbb{k}^{\times}-\{ \pm 1\}$. The Dynkin diagram is

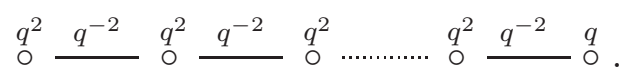

With the notation (1.11) and ordered lexicographically, the positive roots are

$$
\Delta_{+}^{\mathfrak{q}}=\left\{\alpha_{i j} \mid i \leq j \in \mathbb{I}\right\} \cup\left\{\alpha_{i \theta}+\alpha_{j \theta} \mid i<j \in \mathbb{I}\right\} .
$$

The generators of the PBW-basis are $x_{\alpha_{k k}}=x_{k}, x_{\alpha_{k l}}=x_{(k l)}, k<l \in \mathbb{I}_{\theta}$, $x_{\alpha_{i \theta}+\alpha_{\theta}}=\left[x_{\alpha_{i \theta}}, x_{\theta}\right]_{c}, i \in \mathbb{I}_{\theta-1}, x_{\alpha_{i \theta}+\alpha_{j \theta}}=\left[x_{\alpha_{i \theta}+\alpha_{(j+1) \theta}}, x_{j}\right]_{c}, i<j \in \mathbb{I}_{\theta-1}$.

For simplicity, we assume that either $N>4$ is odd or else is $\infty$. The defining relations are

$$
\begin{aligned}
x_{i j} & =0, & i<j-1 ; & x_{i i i \pm 1}=0, \quad i<\theta ; \\
x_{\theta \theta \theta \theta-1} & =0 ; & & \\
x_{\alpha}^{N} & =0, & \alpha \in \Delta_{+} . &
\end{aligned}
$$

where $i, j \in \mathbb{I}$. See [AA2, $\S 4.2]$ for the relations in other cases. As usual we omit (4.37) when $q \notin \mathbb{G}_{\infty}$.

4.4.1. $\mathbb{J}=\{1\}$. The algebra $\mathscr{B}\left(Z_{U}\right)$ is presented by $x_{12}, x_{2}, x_{3}, \ldots, x_{\theta}$ with defining relations (4.35), $i, j \in \mathbb{I}_{2, \theta}$; (4.36); (4.37), $\operatorname{supp} \alpha \subset \mathbb{I}_{2, \theta}$; (4.24); (4.25); (4.26); (4.27); (4.28); and the new relations

$$
\left(\left[\cdots\left[\left[x_{12}, x_{(3 \theta)}\right]_{c}, x_{\theta}\right]_{c}, \cdots, x_{l}\right]_{c}\right)^{N}=0, \quad l \in \mathbb{I}_{2, \theta} .
$$

4.4.2. $\mathbb{J}=\{\theta\}$. The algebra $\mathscr{B}\left(Z_{U}\right)$ is presented by $x_{1}, x_{2}, \ldots, x_{\theta-1}, u_{1}=$ $x_{\theta \theta-1}, u_{2}=x_{\theta \theta \theta-1}$, with defining relations (4.35), $i, j \in \mathbb{I}_{\theta}$; (4.37), $\alpha=\alpha_{k l}$, $k<l \in \mathbb{I}_{\theta-1} ;$ and the new relations

$$
\begin{aligned}
& {\left[x_{i}, u_{1}\right]_{c}=0, \quad\left[x_{i}, u_{2}\right]_{c}=0, \quad i \in \mathbb{I}_{\theta-3} ;} \\
& {\left[\left[x_{\theta-2}, u_{1}\right]_{c}, u_{1}\right]_{c}=-q_{\theta \theta-1}\left[x_{\theta-2} \theta-1, u_{2}\right]_{c} ;} \\
& {\left[\left[x_{\theta-2}, u_{2}\right]_{c}, u_{1}\right]_{c}=0, \quad\left[\left[x_{\theta-2}, u_{2}\right]_{c}, u_{2}\right]_{c}=0 ;} \\
& {\left[x_{(i \theta-2)}, u_{1}\right]_{c}^{N}=0, \quad\left[x_{(i \theta-2)}, u_{2}\right]_{c}^{N}=0, \quad i \in \mathbb{I}_{\theta-2} ;} \\
& {\left[\ldots\left[\left[x_{(i \theta-2)}, u_{2}\right]_{c}, x_{\theta-1}\right]_{c}, \ldots x_{j}\right]_{c}^{N}=0, \quad i<j \in \mathbb{I}_{\theta-2} ;} \\
& u_{1}^{N}=0 ; \quad u_{2}^{N}=0 .
\end{aligned}
$$


4.4.3. $\mathbb{J}=\mathbb{I}_{\theta-1}$. Here, $Z_{U}$ is spanned by $z_{i}:=x_{(i \theta)}, i \in \mathbb{I}$.

The algebra $\mathscr{B}\left(Z_{U}\right)$ is presented by $z_{i}, i \in \mathbb{I}$, with defining relations

$$
\begin{aligned}
& {\left[z_{i},\left[z_{i}, z_{j}\right]_{c}\right]_{c}=0, \quad\left[\left[z_{i}, z_{j}\right]_{c}, z_{j}\right]_{c}=0, \quad i<j \in \mathbb{I} ;} \\
& {\left[\left[z_{i}, z_{j}\right]_{c}, z_{k}\right]_{c}=0, \quad\left[\left[z_{i}, z_{k}\right]_{c}, z_{j}\right]_{c}=0, \quad i<j<k \in \mathbb{I} ;} \\
& z_{i}^{N}=0, \quad i \in \mathbb{I} ; \quad\left[z_{i}, z_{j}\right]_{c}^{N}=0, i<j \in \mathbb{I} .
\end{aligned}
$$

4.4.4. $\mathbb{J}=\mathbb{I}_{2, \theta}$. Here, $Z_{U}$ is spanned by

$$
w_{i}:=x_{i i-1 \ldots 1}, i \in \mathbb{I}, \quad \text { and } \quad \widetilde{w}_{j}:=x_{j \ldots \theta \theta \ldots 1}, j \in \mathbb{I}_{2, \theta} .
$$

The algebra $\mathscr{B}\left(Z_{U}\right)$ is presented by $w_{i}, \widetilde{w}_{i}, i \in \mathbb{I}$, with defining relations

$$
\begin{array}{ll}
{\left[w_{i}, w_{j}\right]_{c}=0, i<j \in \mathbb{I} ;} & {\left[\widetilde{w}_{i}, \widetilde{w}_{j}\right]_{c}=0, i<j \in \mathbb{I}_{2, \theta} ;} \\
{\left[\widetilde{w}_{j}, w_{j-1}\right]_{c}=q_{j-1} q(q-1) w_{\theta}^{2},} & j \in \mathbb{I}_{2, \theta} ; \\
{\left[\widetilde{w}_{j}, w_{i}\right]_{c}=0,} & i \in \mathbb{I}, j \in \mathbb{I}_{2, \theta}-\{i+1\} ; \\
w_{i}^{N}=0, i \in \mathbb{I} ; \quad \widetilde{w}_{j}^{N}=0, j \in \mathbb{I}_{2, \theta} .
\end{array}
$$

4.5. Cartan type $C_{\theta}, \theta>2$. Here $q \in \mathbb{k}^{\times}-\{ \pm 1\}$. The Dynkin diagram is

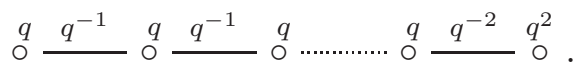

The set of positive roots is

$$
\Delta^{+}=\left\{\alpha_{i j} \mid i \leq j \in \mathbb{I}\right\} \cup\left\{\alpha_{i \theta}+\alpha_{j \theta-1} \mid i \leq j \in \mathbb{I}_{\theta-1}\right\} .
$$

The generators of the PBW-basis are $x_{\alpha_{k k}}=x_{k}, x_{\alpha_{i j}}=x_{(i j)}, i<j \in \mathbb{I}$, $x_{\alpha_{i \theta}+\alpha_{i \theta-1}}=\left[x_{(i \theta)}, x_{(i \theta-1)}\right]_{c}, x_{\alpha_{i \theta}+\alpha_{\theta-1}}=\left[x_{(i \theta)}, x_{\theta-1}\right]_{c}, i \in \mathbb{I}_{\theta-1}, x_{\alpha_{i \theta}+\alpha_{j \theta-1}}=$ $\left[x_{\alpha_{i \theta}+\alpha_{j+1 \theta-1}}, x_{j}\right]_{c}, i<j \in \mathbb{I}_{\theta-2}$. For simplicity, we assume that either $N>4$ is odd or else is $\infty$. The defining relations are

$$
\begin{array}{lc}
x_{i j}=0, \quad i<j-1 ; & x_{i i j}=0, \quad j=i \pm 1,(i, j) \neq(\theta-1, \theta) ; \\
x_{i i i \theta}=0, \quad i=\theta-1 ; & \alpha \in \Delta_{+} .
\end{array}
$$

See [AA2, §4.2] for the relations in other cases. As usual we omit (4.52) when $q \notin \mathbb{G}_{\infty}$.

4.5.1. $\mathbb{J}=\mathbb{I}_{\theta-1}$. Here, $Z_{U}$ is spanned by

$$
z_{i}:=x_{(i \theta)}, i \in \mathbb{I}, \quad \text { and } \quad y_{i j}:=\left[x_{(i \theta-1)}, x_{(j \theta)}\right]_{c}, i \leq j \in \mathbb{I}_{\theta-1} .
$$


The algebra $\mathscr{B}\left(Z_{U}\right)$ is presented by generators $z_{i}, i \in \mathbb{I}$, and $y_{i j}, i<j \in \mathbb{I}_{\theta-1}$, with defining relations

$$
\begin{aligned}
& {\left[z_{i}, z_{j}\right]_{c}=0, \quad i<j \in \mathbb{I}} \\
& {\left[y_{i i}, z_{i}\right]_{c}=0, \quad\left[y_{i i}, z_{\theta}\right]_{c}=\mathfrak{q}_{\alpha_{(i \theta)} \alpha_{\theta}}\left(1-q^{-1}\right) z_{i}^{2}, \quad i \in \mathbb{I}_{\theta-1} ;} \\
& {\left[y_{i j}, z_{\theta}\right]_{c}=\mathfrak{q}_{\alpha_{(i \theta-1)} \alpha_{(j \theta)}}\left(q^{2}-1\right) z_{j} z_{i}, \quad i<j \in \mathbb{I}_{\theta-1} ;} \\
& {\left[y_{i j}, z_{k}\right]_{c}=\mathfrak{q}_{\alpha_{j \theta} \alpha_{k \theta}}(q-1) z_{j} y_{i k}, \quad i \leq j<k \in \mathbb{I}_{\theta-1} \text {; }} \\
& {\left[y_{i k}, z_{j}\right]_{c}=0, \quad\left[z_{i}, y_{j k}\right]_{c}=0, \quad i \leq j \leq k \in \mathbb{I}_{\theta-1} \text {; }} \\
& {\left[y_{i j}, y_{k l}\right]_{c}=0, \quad i, j, k, l \in \mathbb{I}_{\theta-1} \text {; }}
\end{aligned}
$$

$$
z_{i}^{N}=0, i \in \mathbb{I} ; \quad y_{i j}^{N}=0, i \leq j \in \mathbb{I}_{\theta-1} .
$$

4.5.2. $\mathbb{J}=\mathbb{I}_{2, \theta}$. Here, $Z_{U}$ is spanned by

$$
w_{i}:=x_{i i-1 \ldots 1}, i \in \mathbb{I}, \quad \text { and } \quad \widetilde{w}_{j}:=\left[x_{\theta \ldots j}, w_{\theta-1}\right]_{c}, j \in \mathbb{I}_{\theta-1} .
$$

The algebra $\mathscr{B}\left(Z_{U}\right)$ is presented by generators $w_{i}, \widetilde{w}_{i}, i \in \mathbb{I}$, with defining relations

$$
\begin{aligned}
& {\left[w_{i}, w_{j}\right]_{c}=0, \quad i<j \in \mathbb{I}, \quad(i, j) \neq(\theta-1, \theta)} \\
& {\left[w_{\theta-1},\left[w_{\theta-1}, w_{\theta}\right]_{c}\right]_{c}=\left[w_{\theta},\left[w_{\theta}, w_{\theta-1}\right]_{c}\right]_{c}=0} \\
& {\left[\widetilde{w}_{i}, \widetilde{w}_{j}\right]_{c}=0, \quad i<j \in \mathbb{I}_{\theta-1} ;} \\
& {\left[\widetilde{w}_{j}, w_{j-1}\right]_{c}=\mathfrak{q}_{\alpha(j \theta)} \alpha_{(1 \in-1)}(q-1) w_{\theta} w_{\theta-1}, \quad j \in \mathbb{I}_{\theta-1} ;} \\
& {\left[\widetilde{w}_{j}, w_{i}\right]_{c}=0, \quad i \in \mathbb{I}, j \in \mathbb{I}_{\theta-1}-\{i+1\} ;} \\
& w_{i}^{N}=0, \quad i \in \mathbb{I} ; \quad \widetilde{w}_{j}^{N}=0, j \in \mathbb{I}_{\theta-1} ; \quad\left[w_{\theta}, w_{\theta-1}\right]_{c}^{N}=0 .
\end{aligned}
$$

4.6. Cartan type $D_{\theta}, \theta>3$. Here $q \in \mathbb{k}^{\times}-\{1\}$. The Dynkin diagram is

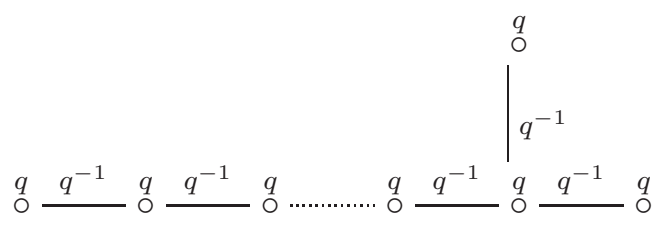

With the notation (1.11) and ordered lexicographically, the positive roots are

$$
\begin{aligned}
& \Delta^{+}=\left\{\alpha_{i j} \mid i \leq j \in \mathbb{I},(i, j) \neq(\theta-1, \theta)\right\} \\
& \cup\left\{\alpha_{i \theta-2}+\alpha_{\theta} \mid i \in \mathbb{I}_{\theta-2}\right\} \cup\left\{\alpha_{i \theta}+\alpha_{j \theta-2} \mid i<j \in \mathbb{I}_{\theta-2}\right\} .
\end{aligned}
$$

The generators of the PBW-basis are

$$
\begin{aligned}
x_{\alpha_{k k}} & =x_{k}, \quad k \in \mathbb{I}, & x_{\alpha_{i j}} & =x_{(i j)}, \quad i<j \in \mathbb{I}_{\theta-1}, \\
x_{\alpha_{i \theta-2}+\alpha_{\theta}} & =\left[x_{(i \theta-2)}, x_{\theta}\right]_{c}, & x_{\alpha_{i \theta}} & =\left[x_{\alpha_{i \theta-2}+\alpha_{\theta}}, x_{\theta-1}\right]_{c}, \quad i \in \mathbb{I}_{\theta-2}, \\
x_{\alpha_{i \theta}+\alpha_{j \theta-2}} & =\left[x_{\alpha_{i \theta}+\alpha_{j+1 \theta-2}}, x_{j}\right]_{c}, & i & <j \in \mathbb{I}_{\theta-2} .
\end{aligned}
$$


For simplicity, we assume that either $N>2$ or else is $\infty$. The defining relations are

$$
\begin{aligned}
& x_{(\theta-1) \theta}=0 ; \quad x_{i j}=0, \quad i<j-1,(i, j) \neq(\theta-2, \theta) ; \\
& x_{i i \theta}=0, i=\theta-2 ; \quad x_{i i j}=0, \quad|j-i|=1, i, j \neq \theta ; \\
& x_{i i(\theta-2)}=0, i=\theta ; \quad x_{\alpha}^{N}=0, \quad \alpha \in \Delta_{+} .
\end{aligned}
$$

See [AA2, §4.2] for the relations in other cases. As usual we omit the last set of relations when $q \notin \mathbb{G}_{\infty}$.

4.6.1. $\mathbb{J}=\mathbb{I}_{\theta-1}$. Here, $Z_{U}$ is spanned by $x_{\theta}$,

$$
\begin{array}{rlrl}
z_{i} & :=\left[x_{(i \theta-2)}, x_{\theta}\right], & \widetilde{z}_{i}:=\left[x_{\theta-1}, z_{i}\right]_{c}, & i \in \mathbb{I}, \\
y_{i j} & :=\left[x_{(i \theta-1)}, z_{j}\right]_{c}, & i<j \in \mathbb{I}_{\theta-2} .
\end{array}
$$

The algebra $\mathscr{B}\left(Z_{U}\right)$ is presented by generators $x_{\theta}, z_{i}, \widetilde{z}_{i}, i \in \mathbb{I}$, and $y_{i j}$, $i<j \in \mathbb{I}_{\theta-1}$, with defining relations

$$
\begin{array}{ll}
{\left[z_{i}, z_{j}\right]_{c}=0, \quad\left[\widetilde{z}_{i}, \widetilde{z}_{j}\right]_{c}=0, \quad\left[\widetilde{z}_{i}, z_{j}\right]_{c}=0,} & i<j \in \mathbb{I}_{\theta-2} ; \\
{\left[z_{i}, \widetilde{z}_{j}\right]_{c}=\mathfrak{q}_{(i j-1)}, \alpha_{(j \theta-2)}+\alpha_{\theta}(q-1) z_{j} \widetilde{z}_{i},} & i<j \in \mathbb{I}_{\theta-2} ; \\
{\left[z_{i}, x_{\theta}\right]_{c}=0, \quad\left[\widetilde{z}_{i}, x_{\theta}\right]_{c}=0,} & i \in \mathbb{I}_{\theta-2} ; \\
{\left[y_{i j}, z_{\theta}\right]_{c}=\mathfrak{q}_{\alpha_{(i \theta-1)}, \alpha_{(j \theta-2)}+\alpha_{\theta}(q-1) z_{j} \widetilde{z}_{i},}} & i<j \in \mathbb{I}_{\theta-2} ; \\
{\left[y_{i j}, z_{k}\right]_{c}=0, \quad\left[z_{i}, y_{j k}\right]_{c}=0, \quad} & i<j, i \leq k \in \mathbb{I}_{\theta-2} ; \\
{\left[y_{i j}, \widetilde{z}_{k}\right]_{c}=0, \quad\left[\widetilde{z}_{i}, y_{j k}\right]_{c}=0,} & i<j, i \leq k \in \mathbb{I}_{\theta-2} ; \\
{\left[y_{i j}, y_{k l}\right]_{c}=0, \quad} & i, j, l \in \mathbb{I}_{\theta-2} ; \\
x_{\theta}^{N}=0 ; \quad z_{i}^{N}=\widetilde{z}_{i}^{N}=0, i \in \mathbb{I}_{\theta-2} ; \quad y_{i j}^{N}=0, i \leq j \in \mathbb{I}_{\theta-1} .
\end{array}
$$

4.6.2. $\mathbb{J}=\mathbb{I}_{2, \theta}$. Here, $Z_{U}$ is spanned by $\widetilde{w}_{\theta}:=\left[x_{\theta}, w_{\theta-2}\right]$,

$$
w_{i}:=x_{i i-1 \ldots 1}, i \in \mathbb{I}, \quad v_{j}:=\left[\left[x_{\theta}, x_{\theta-2 \ldots j}\right]_{c}, w_{\theta-1}\right]_{c}, j \in \mathbb{I}_{\theta-2} .
$$

The algebra $\mathscr{B}\left(Z_{U}\right)$ is presented by $w_{i}, i \in \mathbb{I}, \widetilde{w}_{\theta}, v_{j}, j \in \mathbb{I}_{\theta-2}$, with defining relations

$$
\begin{aligned}
& {\left[w_{i}, w_{j}\right]_{c}=0, i>j \in \mathbb{I} ; \quad\left[v_{i}, v_{j}\right]_{c}=0, i>j \in \mathbb{I}_{\theta-2} ;} \\
& {\left[\widetilde{w}_{\theta}, w_{i}\right]_{c}=0, i \in \mathbb{I} ; \quad\left[\widetilde{w}_{\theta}, v_{i}\right]_{c}=0, i \in \mathbb{I}_{\theta-2} ;} \\
& {\left[v_{j}, w_{j-1}\right]_{c}=\mathfrak{q}_{\alpha_{(j \theta-2)}+\alpha_{\theta}, \alpha_{(1-1)}}(q-1) w_{\theta-1} \widetilde{w}_{\theta}, \quad j \in \mathbb{I}_{\theta-2} ;} \\
& {\left[v_{j}, w_{i}\right]_{c}=0, \quad i \in \mathbb{I}, j \in \mathbb{I}_{\theta-2}-\{i+1\}} \\
& w_{i}^{N}=0, i \in \mathbb{I} ; \quad v_{j}^{N}=0, \quad j \in \mathbb{I}_{\theta-2} ; \quad \widetilde{w}_{\theta}^{N}=0
\end{aligned}
$$

\section{Decompositions With a Block}

Below we follow the paper AAH1. 
5.1. A block and a point, weak interaction. Let $W$ be a braided vector space of dimension 3 with braiding given in the basis $\left(x_{i}\right)_{i \in \mathbb{I}_{3}}$ by

$$
\left(c\left(x_{i} \otimes x_{j}\right)\right)_{i, j \in \mathbb{I}_{3}}=\left(\begin{array}{ccc}
\epsilon x_{1} \otimes x_{1} & \left(\epsilon x_{2}+x_{1}\right) \otimes x_{1} & q_{12} x_{3} \otimes x_{1} \\
\epsilon x_{1} \otimes x_{2} & \left(\epsilon x_{2}+x_{1}\right) \otimes x_{2} & q_{12} x_{3} \otimes x_{2} \\
q_{21} x_{1} \otimes x_{3} & q_{21}\left(x_{2}+a x_{1}\right) \otimes x_{3} & q_{22} x_{3} \otimes x_{3}
\end{array}\right) .
$$

Let $V$, respectively $U$, be the subspace generated by $x_{1}, x_{2}$, respectively $x_{3} ; V$ is a block and $U$ is a point. The scalar $q_{12} q_{21}$ is called the interaction between the block and the point. As in [AAH1, the ghost is $\mathscr{G}= \begin{cases}-2 a, & \epsilon=1, \\ a, & \epsilon=-1 .\end{cases}$ If $\mathscr{G} \in \mathbb{N}$, then we say that the ghost is discrete.

Theorem 5.1. AAH1, 4.1] $\mathscr{B}(W)$ has finite Gelfand-Kirillov dimension if and only if $\epsilon, q_{22}$, the interaction and the ghost are as in [AAH1, Table 5].

From now on, we assume that $\mathrm{GK}-\operatorname{dim} \mathscr{B}(W)<\infty$, i.e. that it is as in [AAH1, Table 5]. Our aim is to compute $Z_{V}=\operatorname{ad}_{c} \mathscr{B}(U)(V)$. Clearly, GK-dim $\mathscr{B}\left(Z_{V}\right)= \begin{cases}\text { GK-dim } \mathscr{B}(W)-1, & q_{22}=1, \\ \text { GK-dim } \mathscr{B}(W), & q_{22} \neq 1 .\end{cases}$

We first deal with the case when the interaction is weak, i.e. that $q_{12} q_{21}=$ 1. Let $y_{i}=x_{i}, i \in \mathbb{I}_{2}$, and

$$
y_{i+2}=\left(\operatorname{ad}_{c} x_{3}\right)^{i} x_{2}, \quad i \in \mathbb{N} .
$$

Lemma 5.2. (1) If $q_{22}= \pm 1$, then $\left(y_{i}\right)_{i \in \mathbb{I}_{3}}$ is a basis of $Z_{V}$, with braid$\operatorname{ing}\left(c\left(y_{i} \otimes y_{j}\right)\right)_{i, j \in \mathbb{I}_{3}}=$

$$
\left(\begin{array}{ccc}
\epsilon y_{1} \otimes y_{1} & \left(\epsilon y_{2}+y_{1}\right) \otimes y_{1} & \epsilon q_{12} y_{3} \otimes y_{1} \\
\epsilon y_{1} \otimes y_{2} & \left(\epsilon y_{2}+y_{1}\right) \otimes y_{2} & \epsilon q_{12} y_{3} \otimes y_{2} \\
\epsilon q_{21} y_{1} \otimes y_{3} & \epsilon q_{21}\left(y_{2}+(a+\epsilon) y_{1}\right) \otimes y_{3}-a \epsilon y_{3} \otimes y_{1} & \epsilon q_{22} y_{3} \otimes y_{3}
\end{array}\right) .
$$

(2) If $q_{22} \in \mathbb{G}_{3}^{\prime}$, then $\left(y_{i}\right)_{i \in \mathbb{I}_{4}}$ is a basis of $Z_{V}$, with braiding given by (5.3) when $i, j \in \mathbb{I}_{3}$, except that

$$
\begin{gathered}
c\left(y_{3} \otimes y_{3}\right)=\epsilon q_{22} y_{3} \otimes y_{3}-\epsilon a q_{12} y_{4} \otimes y_{1} ; \\
c\left(y_{i} \otimes y_{4}\right)=\left(\begin{array}{llll}
q_{12}^{2} y_{4} \otimes y_{1} & q_{12}^{2} y_{4} \otimes y_{2} & q_{12} q_{22}^{2} y_{4} \otimes y_{3} & q_{22} y_{4} \otimes y_{4}
\end{array}\right) .
\end{gathered}
$$

$$
\begin{aligned}
& c\left(y_{4} \otimes y_{i}\right)= \\
& \left(\begin{array}{c}
q_{21}^{2} y_{1} \otimes y_{4} \\
q_{21}^{2}\left(y_{2}+(2 a+1) y_{1}\right) \otimes y_{4}+\left(1-q_{22}^{2}\right) q_{21} y_{3} \otimes y_{3}+a\left(q_{22}-1\right) y_{4} \otimes y_{1} \\
q_{21} q_{22}^{2} y_{3} \otimes y_{4}+\left(q_{22}-1\right) y_{4} \otimes y_{3}
\end{array}\right) .
\end{aligned}
$$


Proof. First, $\operatorname{ad}_{c}\left(x_{3}\right) x_{1}=0$ because the interaction is weak. Thus $Z_{V}$ is generated by $y_{i}, i \in \mathbb{N}$. Observe that $y_{i} \in T^{i-1}(W)$ when $i \geq 2$; thus the non-zero $y_{i}$ 's are linearly independent. Notice that

$$
\begin{aligned}
y_{3}=x_{3} x_{2}-q_{21}\left(x_{2}+a x_{1}\right) x_{3}, \quad y_{4} & =x_{3}^{2} x_{2}-q_{21}(2)_{q_{22}} x_{3} x_{2} x_{3}+q_{21}^{2} q_{22} x_{2} x_{3}^{2} \\
& -q_{21} a(2)_{q_{22}} x_{3} x_{1} x_{3}+2 q_{21}^{2} a q_{22} x_{1} x_{3}^{2} .
\end{aligned}
$$

Observe that $\partial_{1}\left(y_{3}\right)=-a x_{3} \neq 0$, hence $y_{3} \neq 0$. Also,

$$
\partial_{1}\left(y_{4}\right)=a\left(q_{22}-1\right) x_{3}^{2}, \quad \partial_{2}\left(y_{4}\right)=0=\partial_{3}\left(y_{4}\right) .
$$

Hence, if $q_{22}= \pm 1$, then $y_{4}=0$, since $x_{3}^{2}=0$ when $q_{22}=-1$. If $q_{22} \in \mathbb{G}_{3}^{\prime}$, then $x_{3}^{2} \neq 0$ but $x_{3}^{3}=0$, thus $y_{4} \neq 0$ and $y_{5}=0$. Now

$$
\delta\left(y_{1}\right)=g_{1} \otimes y_{1}, \quad \delta\left(y_{2}\right)=g_{1} \otimes y_{2}, \quad \delta\left(y_{3}\right)=g_{1} g_{2} \otimes y_{3}-a x_{3} g_{1} \otimes y_{1} .
$$

From here (5.3) and (5.4) follow using that

$$
g_{1} \cdot y_{3}=\epsilon q_{12} y_{3}, \quad g_{2} \cdot y_{3}=q_{22} q_{21} y_{3} .
$$

We also compute

$$
\begin{aligned}
& \delta\left(y_{4}\right)=g_{1} g_{2}^{2} \otimes y_{4}+\left(1-q_{22}^{2}\right) x_{3} g_{1} g_{2} \otimes y_{3}+a\left(q_{22}-1\right) x_{3}^{2} g_{1} \otimes y_{1} ; \\
& g_{1} \cdot y_{4}=q_{12}^{4} y_{4}, \quad g_{2} \cdot y_{4}=q_{22}^{2} q_{21} y_{4} .
\end{aligned}
$$

Now (5.5), (5.6) follow by direct computation.

Recall that the defining relation of the Jordan plane is

$$
x_{2} x_{1}-x_{1} x_{2}+\frac{1}{2} x_{1}^{2}
$$

while for the super Jordan plane the defining relations are

$$
x_{1}^{2}, \quad x_{2} x_{21}-x_{21} x_{2}-x_{1} x_{21} .
$$

We introduce the elements

$$
\mathrm{z}_{t}:=\left(\operatorname{ad}_{c} y_{2}\right)^{t-1} y_{3}, \quad t \in \mathbb{N} .
$$

They are related with the elements $z_{t}$ for the Nichols algebras in [AAH1, §4]:

$$
\mathbf{z}_{t}:=-\epsilon^{t} q_{12} z_{t}-\delta_{1, t} a q_{12} x_{1} x_{3}, \quad \text { for all } t \in \mathbb{N} .
$$

5.1.1. Case $V=\mathfrak{L}(1, \mathscr{G}), \mathscr{G} \in \mathbb{N}$.

Proposition 5.3. The algebra $\mathscr{B}\left(Z_{V}\right)$ is presented by generators $y_{1}, y_{2}, y_{3}$ and relations (5.7),

$$
\begin{aligned}
y_{1} y_{3} & =q_{12} y_{3} y_{1}, \\
\left(\operatorname{ad}_{c} y_{3}\right)^{2} y_{2} & =0, \\
\mathbf{z}_{\mathscr{G}+1} & =0,
\end{aligned}
$$




$$
\mathbf{z}_{t} \mathbf{z}_{t+1}=q_{12}^{-1} \mathbf{z}_{t+1} \mathbf{z}_{t}, \quad 1 \leq t<\mathscr{G} .
$$

$\mathscr{B}\left(Z_{V}\right)$ has a $P B W$-basis

$$
B=\left\{y_{1}^{m_{1}} y_{2}^{m_{2}} \mathbf{z}_{\mathscr{G}}^{n_{\mathscr{G}}} \ldots \mathbf{z}_{1}^{n_{1}}: m_{i}, n_{j} \in \mathbb{N}_{0}\right\}
$$

hence GK-dim $\mathscr{B}\left(Z_{V}\right)=2+\mathscr{G}$.

Proof. Note that $y_{i}=x_{i}, i=1,2$, determine a braided vector subspace of Jordan type, so (5.7) holds in $\mathscr{B}\left(Z_{V}\right)$, while (5.11) and (5.12) by direct computation. Relations (5.13) and (5.14) are 0 in $\mathscr{B}\left(Z_{V}\right)$ by (5.10) and [AAH1, Lemma 4.13]. Hence the quotient $\widetilde{\mathscr{B}}$ of $T(V)$ by (5.7), (5.11), (5.12), (5.13) and (5.14) projects onto $\mathscr{B}\left(Z_{V}\right)$.

We claim that the subspace $I$ spanned by $B$ is a right ideal of $\widetilde{\mathscr{B}}$. The proof follows as in [AAH1, Proposition 4.16]. As $1 \in I, \widetilde{\mathscr{B}}$ is spanned by $B$.

To prove that $\widetilde{\mathscr{B}} \simeq \mathscr{B}\left(Z_{V}\right)$, it remains to show that $B$ is linearly independent in $\mathscr{B}\left(Z_{V}\right)$. This follows from the decomposition (1.7), i.e.

$$
\mathscr{B}(\mathfrak{L}(1, \mathscr{G})) \simeq \mathscr{B}\left(Z_{V}\right) \# \mathbb{k}\left[x_{3}\right]
$$

and [AAH1, Proposition 4.16]. Then $B$ is a basis of $\mathscr{B}\left(Z_{V}\right)$ and $\widetilde{\mathscr{B}}=\mathscr{B}\left(Z_{V}\right)$. The computation of GK-dim follows from the Hilbert series at once.

Theorem 5.4. The algebra $\mathscr{B}\left(Z_{V}\right)$ is an iterated Ore extension; thus it is strongly noetherian, AS-regular and Cohen-Macaulay domain.

Proof. Analogous to Theorem 4.5 .

5.1.2. Case $V=\mathfrak{L}(-1, \mathscr{G}), \mathscr{G} \in \mathbb{N}$.

Proposition 5.5. The algebra $\mathscr{B}\left(Z_{V}\right)$ is presented by generators $y_{1}, y_{2}, y_{3}$ and relations (5.7), (5.11), (5.12) and

$$
\mathrm{z}_{t}^{2}=0, \quad 1 \leq t \leq \mathscr{G} .
$$

The set

$$
B=\left\{x_{1}^{m_{1}} x_{2}^{m_{2}} \mathbf{z}_{\mathscr{G}}^{n_{\mathscr{G}}} \ldots \mathbf{z}_{1}^{n_{1}}: n_{i} \in\{0,1\}, m_{j} \in \mathbb{N}_{0}\right\}
$$

is a basis of $\mathscr{B}\left(Z_{V}\right)$ and $\mathrm{GK}-\operatorname{dim} \mathscr{B}\left(Z_{V}\right)=2$.

Proof. Analogous to Proposition 5.3 . 
5.1.3. Case $V=\mathfrak{L}_{-}(1, \mathscr{G}), \mathscr{G} \in \mathbb{N}$.

Proposition 5.6. The algebra $\mathscr{B}\left(Z_{V}\right)$ is presented by generators $y_{1}, y_{2}, y_{3}$ and relations (5.8), (5.11) and

$$
\begin{array}{rlrl}
\mathbf{z}_{1+2 \mathscr{G}} & =0, & \\
y_{21} \mathbf{z}_{1} & =q_{12}^{2} \mathbf{z}_{1} y_{21}, & \\
\mathbf{z}_{2 k+1}^{2} & =0, & 0 \leq k<\mathscr{G}, \\
\mathbf{z}_{2 k} \mathbf{z}_{2 k+1} & =q_{12}^{-1} \mathbf{z}_{2 k+1} \mathbf{z}_{2 k}, & & 1 \leq k<\mathscr{G} .
\end{array}
$$

The set

$$
B=\left\{y_{1}^{m_{1}} y_{21}^{m_{2}} y_{2}^{m_{3}} \mathbf{z}_{2 \mathscr{G}}^{n_{2} \mathscr{G}} \ldots \mathbf{z}_{1}^{n_{1}}: m_{1}, n_{2 k+1} \in\{0,1\}, m_{2}, m_{3}, n_{2 k} \in \mathbb{N}_{0}\right\}
$$

is a basis of $\mathscr{B}\left(Z_{V}\right)$ and $\mathrm{GK}-\operatorname{dim} \mathscr{B}\left(Z_{V}\right)=\mathscr{G}+2$.

Proof. Analogous to Proposition 5.3 .

5.1.4. Case $V=\mathfrak{L}_{-}(-1, \mathscr{G}), \mathscr{G} \in \mathbb{N}$.

Proposition 5.7. The algebra $\mathscr{B}\left(Z_{V}\right)$ is presented by generators $y_{1}, y_{2}, y_{3}$ and relations (5.8), (5.11), (5.12), (5.16), (5.17) and

$$
\begin{aligned}
\mathbf{z}_{2 k}^{2} & =0, & & 1 \leq k \leq \mathscr{G}, \\
\mathbf{z}_{2 k-1} \mathbf{z}_{2 k} & =-q_{12}^{-1} \mathbf{z}_{2 k} \mathbf{z}_{2 k-1}, & & 0<k \leq \mathscr{G} .
\end{aligned}
$$

The set

$$
B=\left\{y_{1}^{m_{1}} y_{21}^{m_{2}} y_{2}^{m_{3}} \mathbf{z}_{2 \mathscr{G}}^{n_{2} \mathscr{G}} \ldots \mathbf{z}_{1}^{n_{1}}: m_{1}, n_{2 k} \in\{0,1\}, m_{2}, m_{3}, n_{2 k-1} \in \mathbb{N}_{0}\right\}
$$

is a basis of $\mathscr{B}\left(Z_{V}\right)$ and $\mathrm{GK}-\operatorname{dim} \mathscr{B}\left(Z_{V}\right)=\mathscr{G}+2$.

Proof. Analogous to Proposition 5.3.

5.1.5. Case $V=\mathfrak{L}(\omega, 1), \omega \in \mathbb{G}_{3}^{\prime}$.

Proposition 5.8. The algebra $\mathscr{B}\left(Z_{V}\right)$ is presented by generators $y_{1}, y_{2}, y_{3}, y_{4}$ and relations (5.7), (5.11) and

$$
\begin{aligned}
y_{1} y_{4} & =q_{12}^{2} y_{4} y_{1}, \\
y_{2} y_{3} & =q_{12} y_{3} y_{2}, \\
y_{4} y_{2} & =q_{21}^{2} y_{2} y_{4}+q_{21}(1-\omega) y_{3}^{2}, \\
y_{3} y_{4} & =q_{12} \omega^{2} y_{4} y_{3}, \\
y_{3}^{3} & =y_{4}^{3}=0 .
\end{aligned}
$$


The set

$$
B=\left\{y_{1}^{m_{1}} y_{2}^{m_{2}} y_{3}^{n_{1}} y_{4}^{n_{2}}: m_{i} \in \mathbb{N}_{0}, 0 \leq n_{j} \leq 2\right\}
$$

is a basis of $\mathscr{B}\left(Z_{V}\right)$ and $\mathrm{GK}-\operatorname{dim} \mathscr{B}\left(Z_{V}\right)=2$.

Proof. Relations (5.7) and (5.11) are 0 in $\mathscr{B}\left(Z_{V}\right)$ as in Proposition 5.3. Now (5.22)-(5.26) follow from [AAH1, Lemmas $4.23 \& 4.24$ ]. Hence the quotient $\widetilde{\mathscr{B}}$ of $T(V)$ by all these relations projects onto $\mathscr{B}\left(Z_{V}\right)$. Since the subspace $I$ spanned by $B$ is a right ideal of $\widetilde{\mathscr{B}}$ and $1 \in I, \widetilde{\mathscr{B}}$ is spanned by $B$. To prove that $\widetilde{\mathscr{B}} \simeq \mathscr{B}\left(Z_{V}\right)$, it remains to show that $B$ is linearly independent in $\mathscr{B}\left(Z_{V}\right)$. This follows from the decomposition

$$
\mathscr{B}(\mathfrak{L}(\omega, 1)) \simeq \mathscr{B}\left(Z_{V}\right) \# \mathbb{k}\left[x_{3}\right]
$$

as in (1.7) and AAH1, Proposition 4.25]. Then $B$ is a basis of $\mathscr{B}\left(Z_{V}\right)$, $\widetilde{\mathscr{B}}=\mathscr{B}\left(Z_{V}\right)$ and GK-dim $\mathscr{B}\left(Z_{V}\right)=2$.

5.2. A block and a point, mild interaction. Here we keep the notation as in the previous Subsection but we assume that the interaction is mild, that is $q_{12} q_{21}=-1$. We consider the unique Nichols algebra of finite GK-dim, called the Cyclop Nichols algebra: here, $\epsilon=q_{22}=-1$. Let

$$
y_{i}=x_{i}, \quad y_{i+2}=\left(\operatorname{ad}_{c} x_{3}\right) x_{i}, \quad i \in \mathbb{I}_{2} .
$$

Lemma 5.9. A basis of $Z_{V}$ is given by $\left(y_{i}\right)_{i \in \mathbb{I}_{4}}$, with braiding

$$
\begin{aligned}
& c\left(y_{i} \otimes y_{j}\right)= \begin{cases}-y_{1} \otimes y_{i}, & j=1, \\
\left(y_{1}-y_{2}\right) \otimes y_{i}, & j=2, \\
-q_{12} y_{3} \otimes y_{i}, & j=3, \\
q_{12}\left(y_{3}-y_{4}\right) \otimes y_{i}, & j=4,\end{cases} \\
& c\left(y_{3} \otimes y_{j}\right)= \begin{cases}-q_{21} y_{1} \otimes y_{3}-2 y_{3} \otimes y_{1}, & j=1,2 ; \\
-q_{21} y_{2} \otimes y_{3}+2\left(y_{3}-y_{4}\right) \otimes y_{1}, & j=2, \\
-y_{3} \otimes y_{3}, & j=3, \\
-y_{4} \otimes y_{3}, & j=4 ;\end{cases} \\
& c\left(y_{4} \otimes y_{j}\right)= \begin{cases}-q_{21} y_{1} \otimes y_{4}-2 y_{3} \otimes y_{2}-y_{3} \otimes y_{1}, & j=1, \\
-q_{21} y_{2} \otimes y_{4}+2\left(y_{3}-y_{4}\right) \otimes\left(2 y_{2}+y_{1}\right), & j=2, \\
-y_{3} \otimes y_{4}, & j=3, \\
-y_{4} \otimes y_{4}, & j=4 .\end{cases}
\end{aligned}
$$


Proof. First, $\left(\operatorname{ad}_{c} x_{3}\right)^{2} x_{i}=0$ because $x_{3}^{2}=0$. Thus $Z_{V}$ is generated by $y_{i}$, $i \in \mathbb{I}_{4}$. We claim that the $y_{i}$ 's are linearly independent. Indeed,

$$
\partial_{1}\left(y_{3}\right)=2 x_{3}, \quad \partial_{2}\left(y_{3}\right)=0, \quad \partial_{1}\left(y_{4}\right)=x_{3}, \quad \partial_{2}\left(y_{4}\right)=2 x_{3},
$$

and $\partial_{i}\left(y_{j}\right)=\delta_{i j}$ for $i, j \in \mathbb{I}_{2}$. Now

$$
\begin{array}{ll}
\delta\left(y_{1}\right)=g_{1} \otimes y_{1}, & \delta\left(y_{3}\right)=g_{1} g_{2} \otimes y_{3}+2 x_{3} g_{1} \otimes y_{1}, \\
\delta\left(y_{2}\right)=g_{1} \otimes y_{2}, & \delta\left(y_{4}\right)=g_{1} g_{2} \otimes y_{3}+2 x_{3} g_{1} \otimes y_{2}+x_{3} g_{1} \otimes y_{1} .
\end{array}
$$

From here (5.28), (5.29) and (5.30) follow by direct computation.

We set $y_{14}=\left(\operatorname{ad}_{c} y_{1}\right) y_{4}=y_{1} y_{4}+q_{12} y_{4} y_{1}-q_{12} y_{3} y_{1}$.

Proposition 5.10. The algebra $\mathscr{B}\left(Z_{V}\right)$ is presented by generators $\left(y_{j}\right)_{j \in \mathbb{I}_{4}}$ and relations (5.8),

$$
\begin{array}{rlrl}
y_{1} y_{3}+q_{12} y_{3} y_{1} & =0, & y_{2} y_{3}+q_{12} y_{3} y_{2} & =-q_{12} y_{14}-q_{12} y_{3} y_{1}, \\
y_{2} y_{4}+q_{12} y_{4} y_{2} & =0, & y_{3} y_{4}+y_{4} y_{3} & =0, \\
y_{14}^{2} & =0, & y_{3}^{2} & =0, \\
& =0 & y_{4}^{2}=0 .
\end{array}
$$

The set

$$
B=\left\{y_{1}^{m_{1}} y_{21}^{m_{2}} y_{2}^{m_{3}} y_{14}^{n_{1}} y_{3}^{n_{2}} y_{4}^{n_{3}}: m_{1}, n_{i} \in\{0,1\}, m_{2}, m_{3}, \in \mathbb{N}_{0}\right\}
$$

is a basis of $\mathscr{B}\left(Z_{V}\right)$ and $\mathrm{GK}-\operatorname{dim} \mathscr{B}\left(Z_{V}\right)=2$.

Proof. All the quadratic relations belong to $\operatorname{ker}(\mathrm{id}+c)$, the quantum symmetrizer of degree 2 , hence they are defining relations of $Z_{V}$. Now the first relation of (5.33) follows from [AAH1, Lemma 4.34]. Hence the quotient $\widetilde{\mathscr{B}}$ of $T(V)$ by (5.31), (5.32) and (5.33) projects onto $\mathscr{B}\left(Z_{V}\right)$. Using these relations we check that the subspace $I$ spanned by $B$ is a right ideal of $\widetilde{\mathscr{B}}$. Since $1 \in I, \widetilde{\mathscr{B}}$ is spanned by $B$.

To prove that $\widetilde{\mathscr{B}} \simeq \mathscr{B}\left(Z_{V}\right)$, it remains to show that $B$ is linearly independent in $\mathscr{B}\left(Z_{V}\right)$. This follows from the decomposition

$$
\mathscr{B}(\mathfrak{L}(\omega, 1)) \simeq \mathscr{B}\left(Z_{V}\right) \# \mathbb{k}\left[x_{3}\right]
$$

as in (1.7) and AAH1, Proposition 4.39]. Then $B$ is a basis of $\mathscr{B}\left(Z_{V}\right)$, $\widetilde{\mathscr{B}}=\mathscr{B}\left(Z_{V}\right)$ and GK-dim $\mathscr{B}\left(Z_{V}\right)=2$.

Acknowledgements. This paper grew from conversations following a talk by Oscar Márquez on joint work in progress with Dirceu Bagio and Gastón A. García at the Colloquium Quantum 17 hosted by the University of Talca (Chile). We thank them for sharing their results as well as María Ronco and María Inés Icaza for hospitality. We also thank Hiroyuki Yamane for pointing out to us the reference [U3]. 
We are grateful to C.D. Ward and H. West (University of Miskatonic, Arkham) for pointing out to us a mistake in the proof of Lemma 3.6.

The main results of this paper were communicated at the XXII Coloquio Latinoamericano de Álgebra (Quito, August 2017); the Reunión Anual de la Unión Matemática Argentina (Buenos Aires, December 2017); the Workshop Métodos Categóricos en Álgebras de Hopf (Maldonado, December 2017); the Workshop Tensor categories, Hopf algebras and quantum groups (Marburg, January 2018).

\section{REFERENCES}

[A1] Andruskiewitsch, N.: Some remarks on Nichols algebras. In "Hopf algebras", Bergen, Catoiu and Chin (eds.), 25-45, M. Dekker, (2004).

[A2] Andruskiewitsch, N.: On finite-dimensional Hopf algebras. Proceedings of the ICM Seoul 2014 Vol. II, 117-141 (2014)

[A3] Andruskiewitsch, N.: An Introduction to Nichols Algebras. In Quantization, Geometry and Noncommutative Structures in Mathematics and Physics. A. Cardona, P. Morales, H. Ocampo, S. Paycha, A. Reyes, eds., pp. 135-195, Springer (2017).

[AA1] Andruskiewitsch, N., Angiono, I.: On Nichols algebras with generic braiding. In Modules and Comodules, Trends in Mathematics. Brzezinski, T.; Gomez Pardo, J.L.; Shestakov, I.; Smith, P.F. (Eds.), pp. 47-64 (2008). ISBN: 978-3-7643-8741-9.

[AA2] Andruskiewitsch, N., Angiono, I.: On Finite dimensional Nichols algebras of diagonal type. Bull. Math. Sci. 7 353-573 (2017).

[AAH1] Andruskiewitsch, N., Angiono, I., Heckenberger, I.: On finite GK-dimensional Nichols algebras over abelian groups. Mem. Amer. Math. Soc., to appear.

[AAH2] Andruskiewitsch, N., Angiono, I., Heckenberger, I.: On Nichols algebras of infinite rank with finite Gelfand-Kirillov dimension. arXiv:1805.12000.

[AC] Andruskiewitsch, N., Cuadra, J.: On the structure of (co-Frobenius) Hopf algebras. J. Noncommut. Geom. 7 83-104 (2013).

[AHS] Andruskiewitsch, N., Heckenberger, I., Schneider, H.-J.: The Nichols algebra of a semisimple Yetter-Drinfeld module, Amer. J. Math. 132 1493-1547 (2010).

[AS1] Andruskiewitsch, N., Schneider, H.-J., Lifting of quantum linear spaces and pointed Hopf algebras of order $p^{3}$, J. Algebra 209 (1998), 658-691.

[AS2] Andruskiewitsch, N., Schneider, H.-J.: Finite quantum groups and Cartan matrices, Adv. Math. 154 1-45 (2000).

[AS3] Andruskiewitsch, N., Schneider, H.-J.: Pointed Hopf algebras. In Recent developments in Hopf algebras Theory, MSRI Publ. 43 1-68, Cambridge Univ. Pr. (2002).

[AS3] Andruskiewitsch, N., Schneider, H.-J.: On the classification of finite-dimensional pointed Hopf algebras, Ann. Math. 171, 375-417 (2010)

[An1] Angiono, I.: A presentation by generators and relations of Nichols algebras of diagonal type and convex orders on root systems. J. Eur. Math. Soc. 17 2643-2671 (2015).

[An2] Angiono, I.: On Nichols algebras of diagonal type. J. Reine Angew. Math. 683 189-251 (2013).

[An3] Angiono, I.: Distinguished pre-Nichols algebras. Transf. Groups 21 1-33 (2016). 
[AnG] Angiono, I., García Iglesias, A.: Liftings of Nichols algebras of diagonal type II. All liftings are cocycle deformations. arXiv:1605.03113.

[AnG2] Angiono, I., García Iglesias, A.: Pointed Hopf algebras: a guided tour to the liftings. arXiv:1807.07154.

[ArS] Artin, M., Schelter, W. F.: Graded algebras of global dimension 3. Adv. Math. 66 171-216 (1987).

[AST] Artin, M., Schelter, W. F., Tate, J.: Quantum deformations of $G L_{n}$. Comm. Pure Appl. Math. 44 879-895 (1991).

[ASZ] Artin, M., Small, L. W. , Zhang, J. J.: Generic flatness for strongly Noetherian algebras. J. Algebra 221 579-610 (1999).

[CL] Cuntz, M., Lentner, S.: A simplicial complex of Nichols algebras. Math. Z. 285 647-683 (2017).

[DoT] Doi, Y., Takeuchi, M.: Multiplication alteration by two-cocycles - the quantum version. Commun. Alg. 22 5715-5732 (1994).

[Dr1] Drinfeld, V. G.: Hopf algebras and the quantum Yang-Baxter equation. Sov. Math. Dokl. 32 256-258 (1985).

[E] Elle, S.: Classification of relation types of Ore extensions of dimension 5. Commun. Alg. 45 1323-1346 (2017).

[GG] García, G. A., Giraldi, J. M. J.: On Hopf Algebras over quantum subgroups. J. Pure Appl. Algebra 223 (2019), 738-768.

[GM] García, G. A., Mastnak, M.: Deformation by cocycles of pointed Hopf algebras over non-abelian groups. Math. Res. Lett. 22 59-92 (2015).

[Gr] Graña, M.: A Freeness Theorem for Nichols Algebras. J. Algebra 231 235-257 (2000).

[H1] Heckenberger, I.: The Weyl groupoid of a Nichols algebra of diagonal type. Invent. Math. 164 175-188 (2006).

[H2] Heckenberger, I.: Classification of arithmetic root systems. Adv. Math. 220 59-124 (2009).

[HS1] Heckenberger, I., Schneider, H.-J.: Right coideal subalgebras of Nichols algebras and the Duflo order on the Weyl groupoid. Israel J. Math. 197 139-187 (2013).

[HS2] Heckenberger, I., Schneider, H.-J.: Yetter-Drinfeld modules over bosonizations of dually paired Hopf algebras. Adv. Math. 244 54-394 (2013).

[HV] Heckenberger, I., Vendramin, L.: A classification of Nichols algebras of semi-simple Yetter-Drinfeld modules over non-abelian groups. J. Eur. Math. Soc. 19 299-356 (2017).

[HY1] Heckenberger, I., Yamane, H.: Drinfel'd doubles and Shapovalov determinants. Rev. Un. Mat. Argentina 51 107-146 (2010).

[HY2] Heckenberger, I., Yamane, H.: A generalization of Coxeter groups, root systems, and Matsumoto's theorem. Math. Z. 259 255-276 (2008).

[HX] Hu, N., Xiong, R.: On families of Hopf algebras without the dual Chevalley property, Rev. Un. Mat. Argentina, 59 (2018), 443-469.

[L] Lusztig, G.: Introduction to quantum groups. Birkhäuser (1993).

[LW] Li, J., Wang, X.: Some five-dimensional Artin-Schelter regular algebras obtained by deforming a Lie algebra. J. Alg. Appl. 15 (04) 1650060 (2016). 
[M] Majid, S.: Doubles of quasitriangular Hopf algebras. Comm. Algebra 19 3061-3073 (1991).

[Mo] Montgomery, S.: Hopf algebras and their actions on rings, CMBS 82, Amer. Math. Soc. (1993).

[NS] Nevins, T. A., Stafford, J. T.: Sklyanin algebras and Hilbert schemes of points. Adv. Math., 210 405-478 (2007).

[PV] Pogorelsky, B., Vay, C.: Verma and simple modules for quantum groups at nonabelian groups. Adv. Math. 301 423-457 (2016).

[RS] Radford, D. E., Schneider, H.-J.: On the simple representations of generalized quantum groups and quantum doubles. J. Algebra 319 3689-3731 (2008).

[R] Rosso, M.: Quantum groups and quantum shuffles. Invent. Math. 133 399-416 (1998).

[S] Schauenburg, P.: Hopf bi-Galois extensions. Comm. Algebra 24 3797-3825 (1996).

[U1] Ufer, S.: PBW bases for a class of braided Hopf algebras. J. Algebra 280 84-119 (2004).

[U2] Ufer, S.: Triangular braidings and pointed Hopf algebras. J. Pure Appl. Algebra $210307-320$ (2007).

[U3] Ufer, S.: Braided Hopf algebras of triangular type. PhD thesis (2004). https://edoc.ub.uni-muenchen.de/2477/1/ufer_stefan.pdf

[X1] Xiong, R.: On Hopf algebras over the unique 12-dimensional Hopf algebra without the dual Chevalley property. Commun. Algebra, to appear.

[X2] Xiong, R.: Finite-dimensional Hopf algebras over the smallest non-pointed basic Hopf algebra. arXiv: 1801.06205.

[X3] Xiong, R.: On Hopf algebras over basic Hopf algebras of dimension 24. arXiv: 1809.03938.

[WW] Wang Q., Wu Q. S.: A class of AS-regular algebras of dimension five, J. Algebra 362 (2012), 117-144.

[ZZ] Zhang, J. J., Zhang, J.: Double extension regular algebras of type. J. Algebra 322 373-409 (2009).

Facultad de Matemática, Astronomía y Física, Universidad Nacional de Córdoba. Ciem - COniCet. Medina Allende s/n (5000) Ciudad Universitaria, Córdoba, Argentina

E-mail address: (andrus|angiono)@famaf.unc.edu.ar 